\title{
Enantiopure 1,4-Benzoxazines via 1,2-Cyclic Sulfamidates. Synthesis of Levofloxacin.
}

\author{
John F. Bower, ${ }^{a}$ Peter Szeto ${ }^{b}$ and Timothy Gallagher ${ }^{a^{*}}$ \\ ${ }^{a}$ School of Chemistry, University of Bristol, Bristol, BS8 1TS, United Kingdom. \\ ${ }^{b}$ Chemical Development, GlaxoSmithKline, Medicines Research Centre, Stevenage, SG1 2NY, United Kingdom.
}

\section{Supporting Information}

\author{
Contents \\ Page $1 \quad$ (A) General Experimental Details \\ Page $3 \quad$ (B) Experimental Procedures \\ $\begin{array}{lll}\text { Page } 20 & \text { (C) References relevant to SI }\end{array}$ \\ Page $21 \quad$ (D) Copies of 1H and 13C NMR spectra
}

\section{(A) General experimental details}

Starting materials sourced from commercial suppliers were used as received. Dry solvents, where necessary, were obtained by distillation using standard procedures or by passage through a column of anhydrous alumina using equipment from Anhydrous Engineering based on the Grubbs' design. Petrol refers to the fraction of petroleum ether boiling in the range of 40-60 ${ }^{\circ} \mathrm{C}$. The removal of solvents in vacuo was achieved using both a Büchi rotary evaporator (bath temperatures up to $40{ }^{\circ} \mathrm{C}$ ) at a pressure of either $15 \mathrm{mmHg}$ (diaphragm pump) or $0.1 \mathrm{mmHg}$ (oil pump), as appropriate, and a high vacuum line at room temperature. Reactions requiring anhydrous conditions were run under an atmosphere of dry nitrogen; glassware, syringes and needles were either flame dried immediately prior to use or placed in an oven $\left(150{ }^{\circ} \mathrm{C}\right)$ for at least $2 \mathrm{~h}$ and allowed to cool either in a desiccator or under an atmosphere of dry nitrogen; liquid reagents, solutions or solvents were added via syringe through rubber septa; solid reagents were added via Schlenk type adapters. Commercially available Merck Kieselgel $60 \mathrm{~F}_{254}$ aluminium backed plates were used for TLC analysis. Visualisation was achieved by either UV fluorescence, acidic $\mathrm{KMnO}_{4}$ solution and heat, ninhydrin stain and heat, ammonium molybdate solution and heat or iodine vapour. Flash column chromatography (FCC) was performed using Fluorochem 60 silica: 230-400 mesh (40-63 $\mu \mathrm{m}$ ). The crude material was applied to the column as a solution in $\mathrm{CH}_{2} \mathrm{Cl}_{2}$ or by preadsorption onto silica, as appropriate. Melting points were determined using a Reichert melting point table and temperature controller and are uncorrected. Optical rotations were measured using a Perkin-Elmer 241 polarimeter. Elemental analysis was performed by the 
University of Bristol microanalytical service. Infra-red spectra were recorded in the range 4000-600 $\mathrm{cm}^{-1}$ on a Perkin Elmer Spectrum either as neat films or solids compressed onto a diamond window. Abbreviations used are: w (weak), m (medium), s (strong) and br (broad). NMR spectra were recorded on a JEOL GX270, JEOL GX400, JEOL Lambda 300, JEOL Eclipse 400, JEOL Eclipse 300 or JEOL Alpha 500 spectrometer. Chemical shifts are quoted in parts per million (ppm); ${ }^{1} \mathrm{H}$ NMR spectra are referenced to TMS or residual protium of the deuterated solvent; ${ }^{13} \mathrm{C}$ NMR are referenced to TMS or the deuterated solvent. Coupling constants $(J)$ are quoted to the nearest $0.5 \mathrm{~Hz}$. Other abbreviations used are: s (singlet), d (doublet), t (triplet), q (quartet), m (multiplet) and br (broad). Assignments of ${ }^{1} \mathrm{H}$ NMR and ${ }^{13} \mathrm{C}$ NMR signals were made where possible, using COSY, DEPT, HMQC and HMBC experiments. Where mixtures of isomers (e.g. diastereomers) have been characterised together, they are referred to as $A$ and $B$. Mass spectra were determined by the University of Bristol mass spectrometry service by either electron impact (EI) or chemical ionisation (CI) using a Fisons VG Analytical Autospec spectrometer, or by electrospray ionisation (ESI) using a Brüker Daltonics Apex IV spectrometer. N.B. Compound numbers used in the Supporting Information correspond to those used in the main paper. 


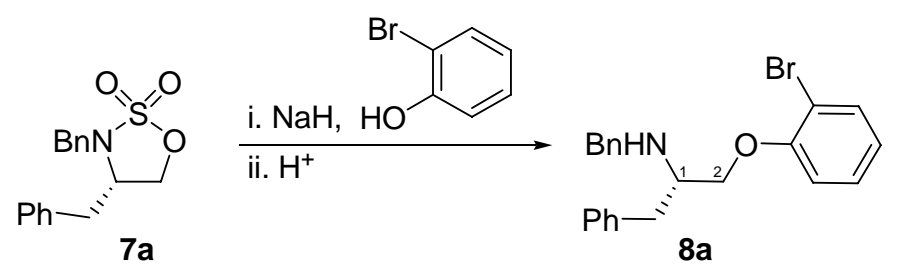

Benzyl[(S)-1-benzyl-2-(2-bromophenoxy)ethyl]amine (8a): To a solution of 2bromophenol (114 mg, $0.66 \mathrm{mmol})$ in anhydrous DMF $(3.8 \mathrm{~mL})$ was added $\mathrm{NaH}(60 \%$ dispersion in mineral oil, $26 \mathrm{mg}, 0.66 \mathrm{mmol}$ ) and the mixture was stirred at r.t. for 15 minutes. Cyclic sulfamidate $7 \mathbf{a}$ (100 mg, $0.33 \mathrm{mmol}$ ) was added and the mixture was stirred at r.t. for a further $16.5 \mathrm{~h}$ prior to the addition of aq. $5 \mathrm{M} \mathrm{HCl}(0.33 \mathrm{~mL})$. The mixture was stirred at r.t. for $2 \mathrm{~h}$, then diluted with aq. $1 \mathrm{M} \mathrm{NaOH}(30 \mathrm{~mL})$ and extracted with $\mathrm{Et}_{2} \mathrm{O}(3 \times 25 \mathrm{~mL})$. The organic extracts were combined, washed with aq. $1 \mathrm{M} \mathrm{NaOH}(3 \times 25 \mathrm{~mL})$, dried $\left(\mathrm{Na}_{2} \mathrm{SO}_{4}\right)$ and concentrated in vacuo. The residue was purified by short FCC (hexanes $100 \%$ - EtOAc$\mathrm{MeOH} 9: 1)$ to afford the product $8 \mathrm{a}(128 \mathrm{mg}, 98 \%)$ as a colourless oil; $[\alpha]_{\mathrm{D}}{ }^{20}+7.9(\mathrm{c}=1.0$, $\mathrm{CHCl}_{3}$ ); $v_{\max } / \mathrm{cm}^{-1}$ (film) 2924 (m), 1481 (s), 1442 (m), 1277 (s), 1248 (m), 1125 (m), 1051 (m), $1030(\mathrm{~s}) ; \delta_{\mathrm{H}}\left(400 \mathrm{MHz}, \mathrm{CDCl}_{3}\right) 2.18(1 \mathrm{H}, \mathrm{br} \mathrm{s}, \mathrm{NH}), 2.94$ (1H, dd, $J=13.5$ and 7.5, C1$\mathrm{C}_{2} \mathrm{Ph}$ ), 2.99 (1H, dd, $J=13.5$ and 6.5, C1- $\left.\underline{\mathrm{C}}_{2} \mathrm{Ph}\right), 3.22-3.29$ (1H, m, C1- $\left.\underline{\mathrm{H}}\right), 3.87$ (1H, dd, $J$ $=9.5$ and 5.5, C2- $\underline{\mathrm{H}}$ ), 3.91-3.95 (3H, m, C2- $\underline{\mathrm{H}}$ and $\left.\mathrm{NC}_{2} \mathrm{Ph}\right), 6.75$ (1H, dd, $J=8.5$ and 1.5, $\operatorname{ArC} \underline{H}), 6.80$ (1H, ddd, $J=8.0,8.0$ and 1.5, $\operatorname{ArC} \underline{H}), 7.16-7.32(11 \mathrm{H}, \mathrm{m}, \operatorname{ArC} \underline{H}), 7.52(1 \mathrm{H}, \mathrm{dd}$,

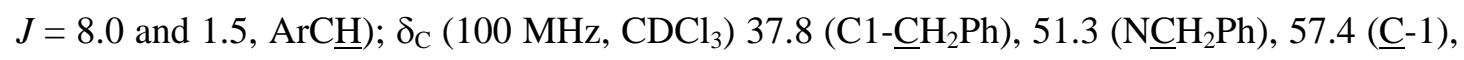
69.8 (ㄷ-2), 112.2 (ArC), 113.0, 121.9, 126.3, 126.9, 128.0 (2 signals), 128.4, 128.5, 129.3 and $133.2(\operatorname{Ar} \underline{C H} \times 14), 138.5,140.2$ and $155.0(\operatorname{Ar} \underline{C} \times 3)$; HRMS: $\left(\mathrm{ESI}^{+}\right)$Found: $[\mathrm{M}+\mathrm{H}]^{+}$ 396.0965, $\mathrm{C}_{22} \mathrm{H}_{23} \mathrm{NO}^{79} \mathrm{Br}$ requires 396.0958.

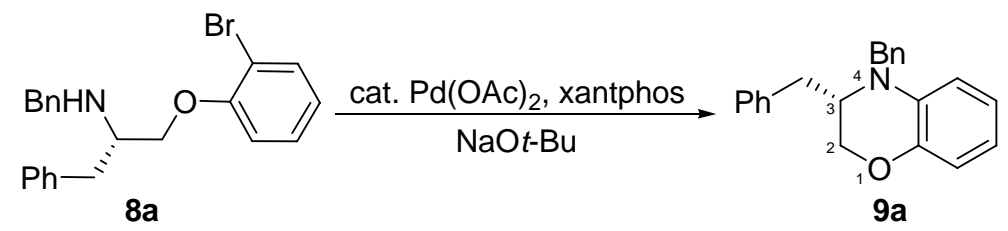

(S)-3,4-Dibenzyl-3,4-dihydro-2H-benzo[1,4]oxazine (9a): $\mathrm{Pd}(\mathrm{OAc})_{2}$ (1.3 mg, $5 \mathrm{~mol} \%$ ), xantphos (3.5 mg, $5 \mathrm{~mol} \%$ ), $t$-BuONa (15 mg, $0.15 \mathrm{mmol}$ ) and amine $8 \mathrm{a}$ (40 mg, $0.10 \mathrm{mmol}$ ) were dissolved in anhydrous PhMe (1.4 mL), placed in a re-sealable tube and heated at $100{ }^{\circ} \mathrm{C}$ for $16 \mathrm{~h}$. The mixture was cooled to r.t., diluted with EtOAc (20 mL), washed with water (10 $\mathrm{mL})$ and then brine $(10 \mathrm{~mL})$, dried $\left(\mathrm{Na}_{2} \mathrm{SO}_{4}\right)$ and concentrated in vacuo. The residue was 
purified by short FCC (EtOAc-hexanes 1:1) to afford benzoxazine 9a (28 mg, $88 \%$ ) as a colourless oil; $[\alpha]_{\mathrm{D}}^{20}+20.5$ (c = 0.4, $\left.\mathrm{CHCl}_{3}\right) ; v_{\max } / \mathrm{cm}^{-1}$ (film) 2923 (m), 1604 (m), 1501 (s), $1453(\mathrm{~m}), 1249(\mathrm{~m}), 1215$ (s); $\delta_{\mathrm{H}}\left(400 \mathrm{MHz}, \mathrm{CDCl}_{3}\right) 2.86(1 \mathrm{H}, \mathrm{dd}, J=13.5$ and 10.0, C3$\mathrm{C}_{2} \mathrm{Ph}$ ), 2.95 (1H, dd, $J=13.5$ and 5.5, C3-C $\underline{\mathrm{H}}_{2} \mathrm{Ph}$ ), 3.46 (1H, dddd, $J=10.0,5.5,2.0$ and 2.0, C3-파), 3.97 (1H, ddd, $J=10.5,2.0$ and 1.0, C2- $\underline{H}$ ), 4.09 (1H, dd, $J=10.5$ and 2.0, C2$\underline{\mathrm{H}}), 4.31\left(1 \mathrm{H}, \mathrm{d}, J=16.5, \mathrm{NC}_{2} \mathrm{Ph}\right), 4.55\left(1 \mathrm{H}, \mathrm{d}, J=16.5, \mathrm{NC}_{2} \mathrm{Ph}\right), 6.59-6.68(2 \mathrm{H}, \mathrm{m}$, $\operatorname{ArC} \underline{\mathrm{H}}), 6.80(1 \mathrm{H}, \mathrm{ddd}, J=6.5,5.5$ and 1.5, $\mathrm{ArC} \underline{\mathrm{H}}), 6.89(1 \mathrm{H}, \mathrm{dd}, J=8.0$ and 1.5, ArC$)$, 7.13-7.34 (10H, m, ArC타); $\delta_{\mathrm{C}}\left(100 \mathrm{MHz}, \mathrm{CDCl}_{3}\right) 35.6\left(\mathrm{C} 3-\underline{\mathrm{CH}}_{2} \mathrm{Ph}\right), 53.7\left(\mathrm{NCH}_{2} \mathrm{Ph}\right), 57.8(\underline{\mathrm{C}}-$ 3), 65.0 (ㄷ-2), 112.7, 116.2, 117.3, 121.9, 126.4, 127.0, 127.2, 128.6, 128.7 and 129.5 (ArCH $\times 14), 134.0,138.1,138.4$ and $143.6(\operatorname{Ar} \underline{C} \times 4)$; HRMS: $\left(\mathrm{ESI}^{+}\right)$Found: $[\mathrm{M}+\mathrm{H}]^{+}$316.1699, $\mathrm{C}_{22} \mathrm{H}_{22} \mathrm{NO}$ requires 316.1696.
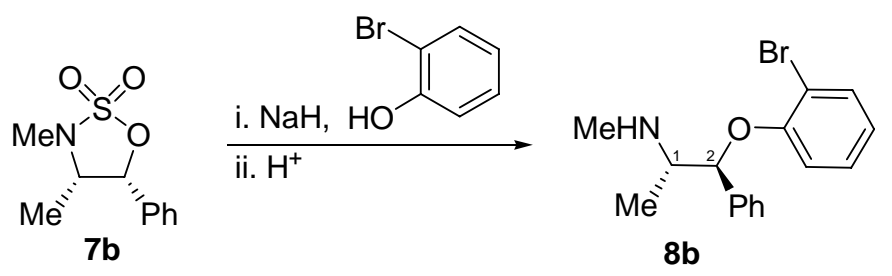

[(1R,2R)-2-(2-Bromophenoxy)-1-methyl-2-phenylethyl]methylamine (8b): To a solution of 2-bromophenol (305 mg, $1.76 \mathrm{mmol})$ in anhydrous DMF (10 mL) was added $\mathrm{NaH}$ (60\% dispersion in mineral oil, $70 \mathrm{mg}, 1.76 \mathrm{mmol}$ ) and the mixture was stirred at r.t. for 15 minutes. Cyclic sulfamidate $\mathbf{7 b}$ (200 mg, $0.88 \mathrm{mmol}$ ) was added and the mixture was stirred at $60{ }^{\circ} \mathrm{C}$ for a further $16 \mathrm{~h}$ prior to the addition of aq. $5 \mathrm{M} \mathrm{HCl}(0.88 \mathrm{~mL})$. The mixture was stirred at r.t. for $2 \mathrm{~h}$, then diluted with aq. $1 \mathrm{M} \mathrm{NaOH}(30 \mathrm{~mL})$ and extracted with $\mathrm{Et}_{2} \mathrm{O}(3 \times 25 \mathrm{~mL})$. The organic extracts were combined, washed with aq. $1 \mathrm{M} \mathrm{NaOH}(3 \times 25 \mathrm{~mL})$, dried $\left(\mathrm{Na}_{2} \mathrm{SO}_{4}\right)$ and concentrated in vacuo. The residue was purified by short FCC (hexanes $100 \%$ EtOAc-MeOH 9:1) to afford the product $8 \mathbf{b}$ (273 mg, $97 \%$ ) as a colourless oil; $[\alpha]_{\mathrm{D}}{ }^{20}-68.3$ (c = 0.8, $\left.\mathrm{CHCl}_{3}\right) ; v_{\max } / \mathrm{cm}^{-1}$ (film) $1585(\mathrm{~m}), 1474(\mathrm{~s}), 1442(\mathrm{~m}), 1274(\mathrm{~m}), 1243(\mathrm{~s}), 1301(\mathrm{~m})$; $\delta_{\mathrm{H}}\left(400 \mathrm{MHz}, \mathrm{CDCl}_{3}\right) 0.95$ (3H, d, $\left.J=6.5, \mathrm{C} 1-\underline{\mathrm{C}}_{3}\right), 2.12(1 \mathrm{H}, \mathrm{br} \mathrm{s}, \underline{\mathrm{H}}), 2.49$ (3H, s, $\left.\mathrm{NC}_{3}\right)$, 3.10 (1H, dq, $J=7.5$ and 6.5, C1- $\underline{\mathrm{H}}), 4.97$ (1H, d, $J=7.5, \mathrm{C} 2-\underline{\mathrm{H}}), 6.67-6.74$ (2H, m, ArC$)$,

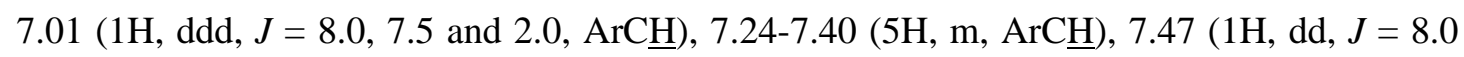
and 2.0, $\mathrm{ArC} \underline{\mathrm{H}}) ; \delta_{\mathrm{C}}\left(100 \mathrm{MHz}, \mathrm{CDCl}_{3}\right) 15.1\left(\mathrm{C} 1-\underline{C H}_{3}\right), 33.4\left(\mathrm{NCH}_{3}\right), 59.8(\underline{\mathrm{C}}-1), 84.9(\underline{\mathrm{C}}-2)$, 112.6 (ArC $), 115.0,121.8,127.2,128.1$ (2 signals), 128.4 and $133.1(\mathrm{Ar} \underline{\mathrm{C}} \mathrm{H} \times$ 9), 138.3 and 154.2 (ArC $\times 2$ ); HRMS: $\left(\mathrm{ESI}^{+}\right)$Found: $[\mathrm{M}+\mathrm{H}]^{+} 320.0648, \mathrm{C}_{16} \mathrm{H}_{19} \mathrm{NO}^{79} \mathrm{Br}$ requires 320.0645 . 


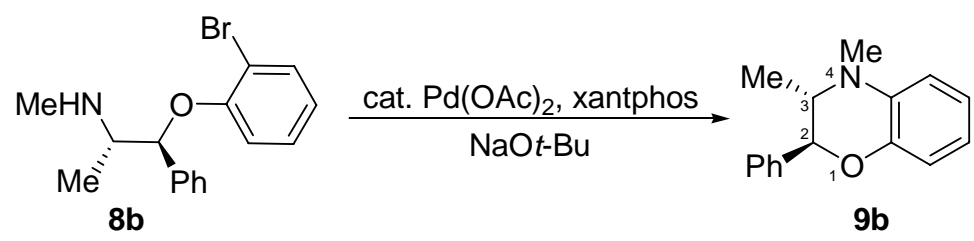

(2R,3R)-3,4-Dimethyl-2-phenyl-3,4-dihydro-2H-benzo[1,4]oxazine (9b): $\mathrm{Pd}(\mathrm{OAc})_{2} \quad(4.0$ mg, $6 \mathrm{~mol} \%$ ), xantphos (9.8 mg, $6 \mathrm{~mol} \%$ ), t-BuONa (39 mg, $0.40 \mathrm{mmol}$ ) and amine $\mathbf{8 b}$ (81 mg, $0.25 \mathrm{mmol}$ ) were dissolved in anhydrous PhMe (3.2 mL), placed in a re-sealable tube and heated at $100{ }^{\circ} \mathrm{C}$ for 40 h. The mixture was cooled to r.t., diluted with EtOAc (20 mL), washed with water $(10 \mathrm{~mL})$ and then brine $(10 \mathrm{~mL})$, dried $\left(\mathrm{Na}_{2} \mathrm{SO}_{4}\right)$ and concentrated in vacuo. The residue was purified by short FCC (EtOAc) to afford benzoxazine $\mathbf{9 b}$ (42 mg, 65 \%) as a pale yellow oil; $[\alpha]_{\mathrm{D}}{ }^{20}-14.3$ (c = 0.6, $\mathrm{CHCl}_{3}$ ); $v_{\max } / \mathrm{cm}^{-1}$ (film) 1606 (m), 1502 (s), 1450 (m), 1260 (s), 1224 (s); $\delta_{\mathrm{H}}\left(400 \mathrm{MHz}, \mathrm{CDCl}_{3}\right) 1.17$ (3H, d, $J=6.5, \mathrm{C} 3-\mathrm{C}_{3}$ ), 2.85 (3H, s, $\left.\mathrm{NC}_{3}\right), 3.43$ (1H, qd, $J=6.5$ and 4.5, C3- $\left.\underline{\mathrm{H}}\right), 4.94$ (1H, d, $\left.J=4.5, \mathrm{C} 2-\underline{\mathrm{H}}\right), 6.64-6.71(2 \mathrm{H}, \mathrm{m}$,

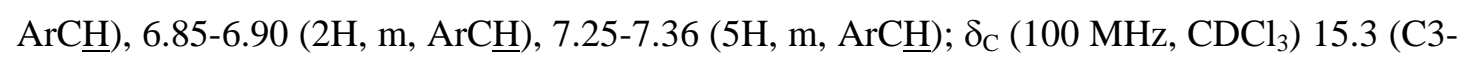
$\left.\underline{\mathrm{CH}}_{3}\right), 36.0$ (C-3), $56.3\left(\mathrm{NCH}_{3}\right), 80.3$ (ㄷ-2), 112.6, 115.8, 117.8, 121.5, 126.6, 127.9 and 128.4 $\left(\mathrm{Ar} \underline{\mathrm{CH}} \times\right.$ 9), 135.5, 140.3 and $143.7\left(\mathrm{Ar} \underline{\mathrm{C}} \times\right.$ 3); HRMS: $\left(\mathrm{ESI}^{+}\right)$Found: $[\mathrm{M}+\mathrm{H}]^{+}$240.1391, $\mathrm{C}_{16} \mathrm{H}_{18} \mathrm{NO}$ requires 240.1382 .
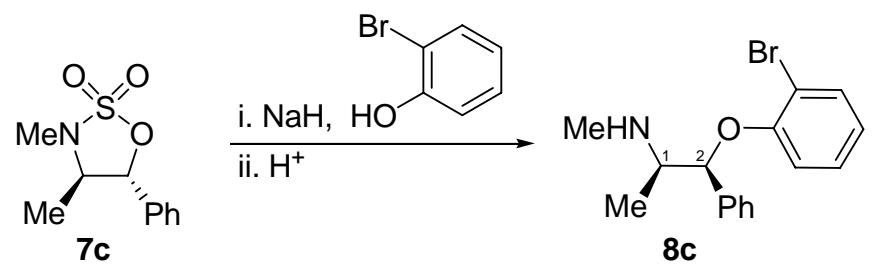

[(1S,2R)-2-(2-Bromophenoxy)-1-methyl-2-phenylethyl]methylamine (8c): To a solution of 2-bromophenol (305 mg, $1.76 \mathrm{mmol})$ in anhydrous DMF (10 mL) was added $\mathrm{NaH}$ (60\% dispersion in mineral oil, $70 \mathrm{mg}, 1.76 \mathrm{mmol}$ ) and the mixture was stirred at r.t. for 5 minutes. Cyclic sulfamidate 7c (200 mg, $0.88 \mathrm{mmol}$ ) was added and the mixture was stirred at $60{ }^{\circ} \mathrm{C}$ for a further $15 \mathrm{~h}$ prior to the addition of aq. $5 \mathrm{M} \mathrm{HCl}(0.88 \mathrm{~mL})$. The mixture was stirred at r.t. for $2 \mathrm{~h}$, then diluted with aq. $1 \mathrm{M} \mathrm{NaOH}(30 \mathrm{~mL})$ and extracted with $\mathrm{Et}_{2} \mathrm{O}(3 \times 25 \mathrm{~mL})$. The organic extracts were combined, washed with aq. $1 \mathrm{M} \mathrm{NaOH}(3 \times 25 \mathrm{~mL})$, dried $\left(\mathrm{Na}_{2} \mathrm{SO}_{4}\right)$ and concentrated in vacuo. The residue was purified by short FCC (hexanes $100 \%$ EtOAc-MeOH 9:1) to afford the product 8c (249 mg, $88 \%$ as a colourless oil; $[\alpha]_{\mathrm{D}}{ }^{20}-100.0$ (c = 1.1, $\left.\mathrm{CHCl}_{3}\right) ; v_{\max } / \mathrm{cm}^{-1}$ (film) 1474 (s), 1442 (m), 1274 (m), 1243 (m), 1030 (m); $\delta_{\mathrm{H}}(400$ $\left.\mathrm{MHz}, \mathrm{CDCl}_{3}\right) 1.17$ (3H, d, $\left.J=6.5, \mathrm{C} 1-\underline{\mathrm{C}}_{3}\right), 1.63$ (1H, br s, $\left.\mathrm{N} \underline{\mathrm{H}}\right), 2.47$ (3H, s, $\mathrm{NC}_{3}$ ), 2.96 (1H, qd, $J=6.5$ and 4.0, C1- $\underline{\mathrm{H}}), 5.31(1 \mathrm{H}, \mathrm{d}, J=4.0, \mathrm{C} 2-\underline{\mathrm{H}}), 6.62(1 \mathrm{H}, \mathrm{dd}, J=8.5$ and 1.5 
$\operatorname{ArC} \underline{H}), 6.74(1 \mathrm{H}, \mathrm{ddd}, J=7.5,7.5$ and 1.0, $\operatorname{ArC} \underline{\mathrm{H}}), 7.03$ (1H, ddd, $J=8.5,7.5$ and 1.5, $\operatorname{ArC} \underline{\mathrm{H}}), 7.24-7.37$ (5H, m, ArC$), 7.51(1 \mathrm{H}, \mathrm{dd}, J=8.0$ and 1.5, $\mathrm{ArC} \underline{\mathrm{H}}) ; \delta_{\mathrm{C}}(100 \mathrm{MHz}$,

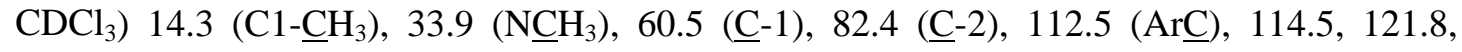
126.4, 127.7, 128.2, 128.5 and 133.2 $\left(\mathrm{ArC} \underline{\mathrm{CH}} \times\right.$ 9), 138.7 and $154.3(\mathrm{ArC} \times 2)$; HRMS: $\left(\mathrm{ESI}^{+}\right)$ Found: $[\mathrm{M}+\mathrm{H}]^{+} 320.0626, \mathrm{C}_{16} \mathrm{H}_{19} \mathrm{NO}^{79} \mathrm{Br}$ requires 320.0624 .
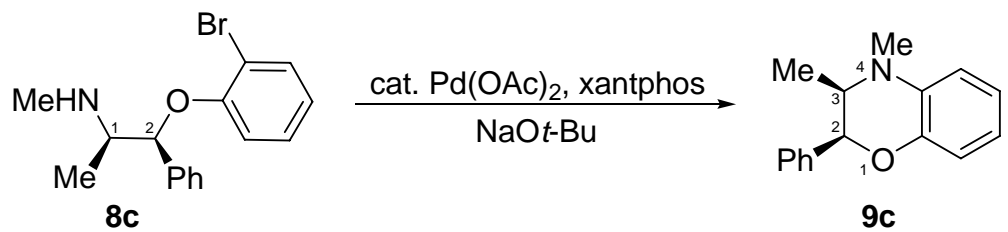

(2R,3S)-3,4-Dimethyl-2-phenyl-3,4-dihydro-2H-benzo[1,4]oxazine (9c): $\mathrm{Pd}(\mathrm{OAc})_{2} \quad$ (4.5 mg, $7 \mathrm{~mol} \%$ ), xantphos (11.4 mg, $7 \mathrm{~mol} \%$ ), $t$-BuONa (45 mg, $0.45 \mathrm{mmol}$ ) and amine 8c (91 $\mathrm{mg}, 0.28 \mathrm{mmol}$ ) were dissolved in anhydrous PhMe $(4.2 \mathrm{~mL})$, placed in a re-sealable tube and heated at $100{ }^{\circ} \mathrm{C}$ for $24 \mathrm{~h}$. The mixture was cooled to r.t., diluted with EtOAc (20 mL), washed with water $(10 \mathrm{~mL})$ and then brine $(10 \mathrm{~mL})$, dried $\left(\mathrm{Na}_{2} \mathrm{SO}_{4}\right)$ and concentrated in vacuo. The residue was purified by short FCC (EtOAc-hexanes 1:1) to afford benzoxazine 9c (53 mg, $79 \%$ ) as a pale yellow oil; $[\alpha]_{\mathrm{D}}{ }^{20}+196.2$ (c = 0.5, $\left.\mathrm{CHCl}_{3}\right) ; v_{\max } / \mathrm{cm}^{-1}$ (film) 1608 (m), 1503 (s), 1450 (m), 1260 (s), 1224 (s), 1130 (m); $\delta_{\mathrm{H}}\left(400 \mathrm{MHz}, \mathrm{CDCl}_{3}\right) 0.82$ (3H, d, J = 6.5, C3- $\left.\underline{\mathrm{H}}_{3}\right), 2.91$ (3H, s, $\mathrm{NC}_{3}$ ), 3.51 (1H, qd, $J=6.5$ and 2.0, C3- $\left.\underline{\mathrm{H}}\right), 5.32$ (1H, d, $J=2.0$,

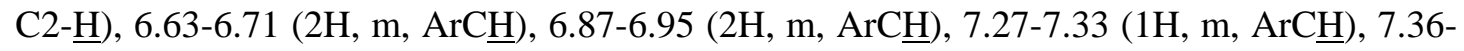

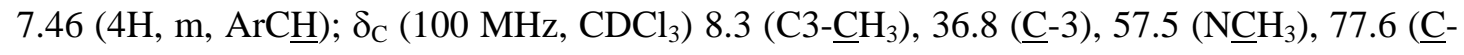
2), 112.2, 115.8, 117.4, 121.8, 125.8, 127.5 and $128.3(\mathrm{Ar} \underline{\mathrm{CH}} \times$ 9), 134.5, 138.7 and 143.8 $(\mathrm{ArC} \times 3)$; HRMS: $\left(\mathrm{ESI}^{+}\right)$Found: $[\mathrm{M}+\mathrm{H}]^{+}$240.1391, $\mathrm{C}_{16} \mathrm{H}_{18} \mathrm{NO}$ requires 240.1383.

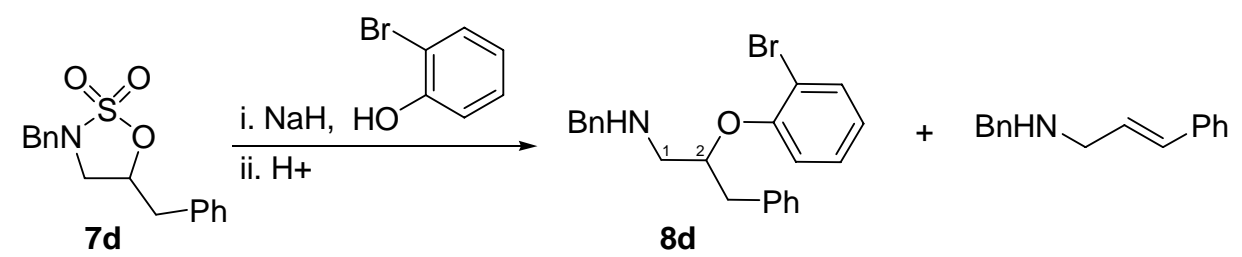

Benzyl[2-(2-bromophenoxy)-3-phenylpropyl]amine (8d) and Benzyl((E)-3-phenylallyl)amine: To a solution of 2-bromophenol (340 mg, $1.96 \mathrm{mmol}$ ) in anhydrous DMF (11 mL) was added $\mathrm{NaH}$ (60 \% dispersion in mineral oil, $78 \mathrm{mg}, 1.96 \mathrm{mmol}$ ) and the mixture was stirred at r.t. for 10 minutes. Cyclic sulfamidate $7 \mathbf{d}$ ( $297 \mathrm{mg}, 0.98 \mathrm{mmol}$ ) was added and the mixture was stirred at $60{ }^{\circ} \mathrm{C}$ for a further $16 \mathrm{~h}$ prior to the addition of aq. $5 \mathrm{M} \mathrm{HCl}(0.98 \mathrm{~mL})$. The mixture was stirred at r.t. for $1 \mathrm{~h}$, then diluted with aq. $1 \mathrm{M} \mathrm{NaOH}(30 \mathrm{~mL})$ and extracted 
with $\mathrm{Et}_{2} \mathrm{O}(3 \times 25 \mathrm{~mL})$. The organic extracts were combined, washed with aq. $1 \mathrm{M} \mathrm{NaOH}(3 \times$ $25 \mathrm{~mL}$ ), dried $\left(\mathrm{Na}_{2} \mathrm{SO}_{4}\right)$ and concentrated in vacuo. The residue was purified by FCC (EtOAchexanes 1:1 - EtOAc-MeOH 9:1) to afford the product 8d (221 mg, 57 \%) as a colourless oil. Continued elution provided N-benzyl cinnamylamine (49 mg, $22 \%$ ) as a colourless oil.

Data for 8d: $v_{\max } / \mathrm{cm}^{-1}$ (film) 1584 (m), 1473 (s), 1441 (m), 1272 (m), 1243 (s), $1029(\mathrm{~m}) ; \delta_{\mathrm{H}}$

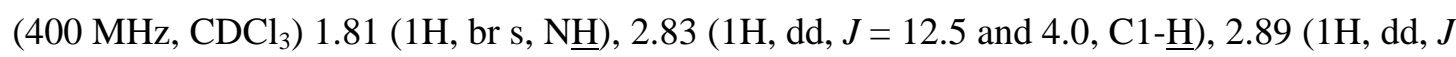
$=12.5$ and 6.5, C1- $\underline{\mathrm{H}}), 3.00$ (1H, dd, $J=13.5$ and 7.0, C2- $\left.\underline{\mathrm{H}}_{2} \mathrm{Ph}\right), 3.11(1 \mathrm{H}, \mathrm{dd}, J=13.5$ and 5.5, C2- $\underline{\mathrm{H}}_{2} \mathrm{Ph}$ ), 3.74 (1H, d, $\left.J=13.5, \mathrm{NCH}_{2} \mathrm{Ph}\right), 3.80$ (1H, d, $\left.J=13.5, \mathrm{NCH}_{2} \mathrm{Ph}\right), 4.57-4.64$ (1H, m, C2- $\underline{\mathrm{H}}), 6.79(1 \mathrm{H}$, ddd, $J=7.5,7.5$ and 1.0, $\mathrm{ArC} \underline{\mathrm{H}}), 6.83(1 \mathrm{H}, \mathrm{dd}, J=8.5$ and 1.5, $\operatorname{ArC} \underline{H})$, 7.14-7.32 (11H, m, ArC프), $7.51(1 \mathrm{H}, \mathrm{dd}, J=8.0$ and 1.5, $\operatorname{ArC} \underline{\mathrm{H}}) ; \delta_{\mathrm{C}}(100 \mathrm{MHz}$,

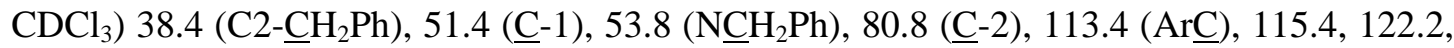
126.5, 126.8, 128.0, 128.3 (2 signals), 128.4, 129.5 and 133.4 (Ar대 $\times 14), 137.5,140.2$ and 154.6 (ArC $\times 3$ ); HRMS: $\left(\mathrm{ESI}^{+}\right)$Found: $[\mathrm{M}+\mathrm{H}]^{+}$396.0950, $\mathrm{C}_{22} \mathrm{H}_{23} \mathrm{NO}^{79} \mathrm{Br}$ requires 396.0958.

Data for $N$-benzyl cinnamylamine: $\delta_{\mathrm{H}}\left(400 \mathrm{MHz}, \mathrm{CDCl}_{3}\right) 1.63(1 \mathrm{H}$, br s, $\mathrm{N} \underline{\mathrm{H}}), 3.42(2 \mathrm{H}, \mathrm{d}, J$ $=6.0, \mathrm{C} 1-\underline{\mathrm{H}}), 3.82\left(2 \mathrm{H}, \mathrm{s}, \mathrm{NC}_{2} \mathrm{Ph}\right), 6.30(1 \mathrm{H}, \mathrm{d} \mathrm{t}, J=16.0$ and 6.0, C2- $\underline{\mathrm{H}}), 6.53(1 \mathrm{H}, \mathrm{d}, J=$ 16.0, C3- $\underline{\mathrm{H}}), 7.18-7.39$ (10H, m, ArCH$) ; ~ m / z ~\left(\mathrm{CI}^{+}\right) 224$ ([M+H] $]^{+}, 100$ \%). The spectroscopic properties of this compound were consistent with the data available in the literature. ${ }^{1}$

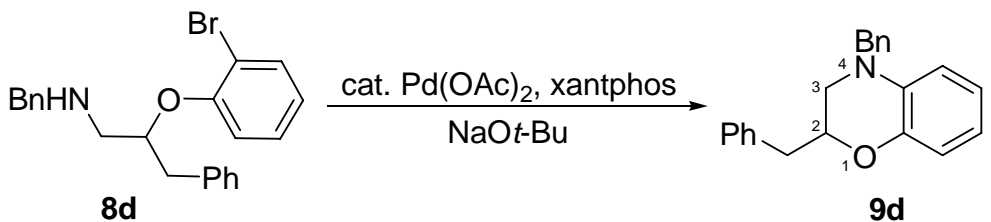

4-Benzyl-2-phenyl-3,4-dihydro-2H-benzo[1,4]oxazine (9d): $\mathrm{Pd}(\mathrm{OAc})_{2}$ (3.0 mg, $7 \mathrm{~mol} \%$ ), xantphos (7.7 mg, $7 \mathrm{~mol} \%$ ), $t$-BuONa (29 mg, $0.30 \mathrm{mmol}$ ) and amine $8 d$ (78 mg, $0.20 \mathrm{mmol}$ ) were dissolved in anhydrous PhMe $(2.8 \mathrm{~mL})$, placed in a re-sealable tube and heated at $100{ }^{\circ} \mathrm{C}$ for $62 \mathrm{~h}$. The mixture was cooled to r.t., diluted with EtOAc $(20 \mathrm{~mL})$, washed with water (10 $\mathrm{mL})$ and then brine $(10 \mathrm{~mL})$, dried $\left(\mathrm{Na}_{2} \mathrm{SO}_{4}\right)$ and concentrated in vacuo. The residue was purified by short FCC (EtOAc-hexanes 2:1) to afford benzoxazine 9d (48 mg, 76 \%) as a pale yellow oil; $v_{\max } / \mathrm{cm}^{-1}$ (film) 1605 (m), 1504 (s), 1453 (m), 1242 (m), 1222 (m); $\delta_{\mathrm{H}}(400 \mathrm{MHz}$, $\left.\mathrm{CDCl}_{3}\right) 2.84\left(1 \mathrm{H}, \mathrm{dd}, J=14.0\right.$ and 7.0, C2- $\left.\underline{\mathrm{H}}_{2} \mathrm{Ph}\right), 3.10-3.19$ (2H, m, C2-C $\underline{H}_{2} \mathrm{Ph}$ and C3- $\left.\underline{\mathrm{H}}\right)$, 3.25 (1H, dd, $J=11.5$ and 2.5, C3- $\underline{\mathrm{H}}), 4.33-4.41$ (2H, m, C2- $\underline{\mathrm{H}}$ and $\left.\mathrm{NC}_{2} \mathrm{Ph}\right), 4.46$ (1H, d, $J$ 
$\left.=16.5, \mathrm{NC}_{2} \mathrm{Ph}\right), 6.61-6.67$ (2H, m, ArC$), 6.74-6.79(1 \mathrm{H}, \mathrm{m}, \operatorname{ArC} \underline{\mathrm{H}}), 6.84$ (1H, dd, $J=8.0$

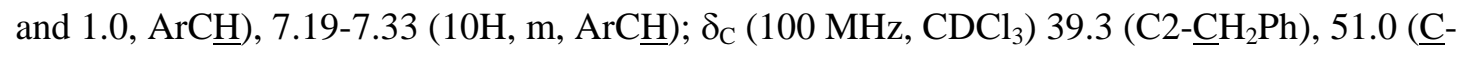
3), 54.9 ( $\left.\mathrm{NCH}_{2} \mathrm{Ph}\right), 74.1$ (--2), 112.3, 116.5, 117.8, 121.5, 126.5, 127.0 (2 signals), 128.5, 128.6 and $129.4(\mathrm{Ar} \underline{\mathrm{C}} \mathrm{H} \times 14), 135.1,137.2,138.1$ and $143.4(\operatorname{Ar} \underline{C} \times 4)$; HRMS: $\left(\mathrm{ESI}^{+}\right)$ Found: $[\mathrm{M}+\mathrm{H}]^{+} 316.1699, \mathrm{C}_{22} \mathrm{H}_{22} \mathrm{NO}$ requires 316.1696.
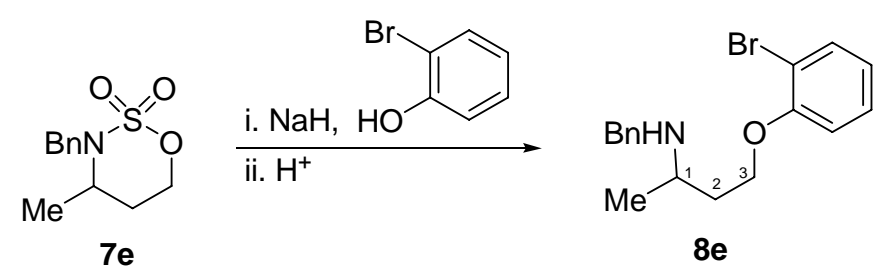

Benzyl[3-(2-bromophenoxy)propyl]amine (8e): To a solution of 2-bromophenol (144 mg, $0.83 \mathrm{mmol})$ in anhydrous DMF $(4.7 \mathrm{~mL})$ was added $\mathrm{NaH}$ (60 \% dispersion in mineral oil, 33 $\mathrm{mg}, 0.83 \mathrm{mmol}$ ) and the mixture was stirred at r.t. for 10 minutes. Cyclic sulfamidate 7e (100 mg, $0.41 \mathrm{mmol}$ ) was added and the mixture was stirred at $60{ }^{\circ} \mathrm{C}$ for a further $15 \mathrm{~h}$ prior to the addition of aq. $5 \mathrm{M} \mathrm{HCl}(0.41 \mathrm{~mL})$. The mixture was stirred at r.t. for $2 \mathrm{~h}$, then diluted with aq. $1 \mathrm{M} \mathrm{NaOH}(30 \mathrm{~mL})$ and extracted with $\mathrm{Et}_{2} \mathrm{O}(3 \times 25 \mathrm{~mL})$. The organic extracts were combined, washed with aq. $1 \mathrm{M} \mathrm{NaOH}(3 \times 25 \mathrm{~mL})$, dried $\left(\mathrm{Na}_{2} \mathrm{SO}_{4}\right)$ and concentrated in vacuo. The residue was purified by short FCC (hexanes $100 \%$ - EtOAc-MeOH 9:1) to afford the product 8e (135 mg, $99 \%$ ) as a colourless oil; $v_{\max } / \mathrm{cm}^{-1}$ (film) 1586 (m), 1470 (s), 1442 (m), $1278(\mathrm{~m}), 1248$ (s), 1051 (m), 1030 (m); $\delta_{\mathrm{H}}\left(400 \mathrm{MHz}, \mathrm{CDCl}_{3}\right) 1.18$ (3H, d, J = 6.5, C1-

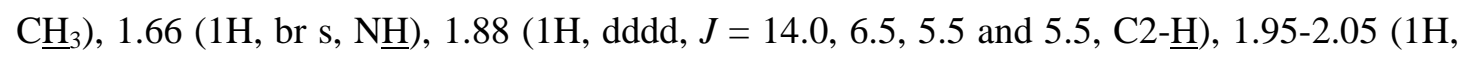
m, C2- $\underline{\mathrm{H}}), 2.97-3.06$ (1H, m, C1- $\underline{\mathrm{H}}), 3.77\left(1 \mathrm{H}, \mathrm{d}, J=13.0, \mathrm{NC}_{2} \mathrm{Ph}\right), 3.88$ (1H, d, $J=13.0$,

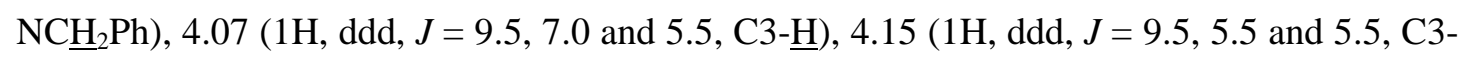
$\underline{\mathrm{H}}), 6.81$ (1H, ddd, $J=7.5,7.5$ and 1.0, $\operatorname{ArC} \underline{\mathrm{H}}), 6.87$ (1H, dd, $J=8.5$ and 1.5, $\operatorname{ArC} \underline{H}), 7.18-$ 7.34 (6H, m, $\operatorname{ArC} \underline{H}), 7.51$ (1H, dd, $J=8.0$ and 1.5, $\mathrm{ArC} \underline{H}) ; \delta_{\mathrm{C}}\left(100 \mathrm{MHz}, \mathrm{CDCl}_{3}\right) 20.6$ (C1-

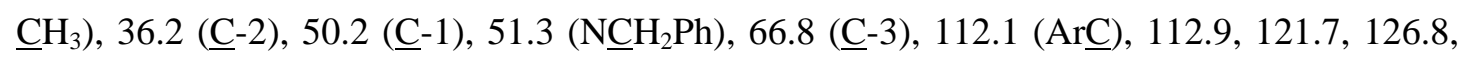
128.1, 128.3 (2 signals) and $133.2(\mathrm{ArCH} \times 9), 140.7$ and $155.2(\operatorname{ArC} \times 2)$; HRMS: $\left(\mathrm{ESI}^{+}\right)$ Found: $[\mathrm{M}+\mathrm{H}]^{+} 334.0801, \mathrm{C}_{17} \mathrm{H}_{21} \mathrm{NO}^{79} \mathrm{Br}$ requires 334.0801 .

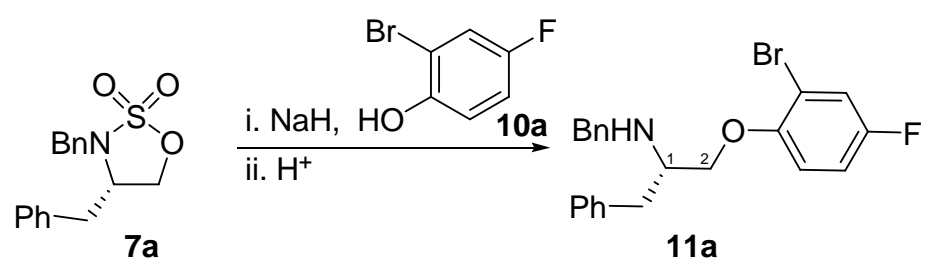


Benzyl[(S)-1-benzyl-2-(2-bromo-4-fluorophenoxy)ethyl]amine (11a): To a solution of 2bromo-4-fluorophenol 10a (126 mg, $0.66 \mathrm{mmol})$ in anhydrous DMF (3.8 mL) was added $\mathrm{NaH}$ (60\% dispersion in mineral oil, $26 \mathrm{mg}, 0.66 \mathrm{mmol}$ ) and the mixture was stirred at r.t. for 5 minutes. Cyclic sulfamidate $7 \mathbf{a}$ (100 mg, $0.33 \mathrm{mmol}$ ) was added and the mixture was stirred at r.t. for a further $23 \mathrm{~h}$ prior to the addition of aq. $5 \mathrm{M} \mathrm{HCl}(0.33 \mathrm{~mL})$. The mixture was stirred at r.t. for 2 h, then diluted with aq. $1 \mathrm{M} \mathrm{NaOH}(30 \mathrm{~mL})$ and extracted with $\mathrm{Et}_{2} \mathrm{O}(3 \times$ $25 \mathrm{~mL})$. The organic extracts were combined, washed with aq. $1 \mathrm{M} \mathrm{NaOH}(3 \times 25 \mathrm{~mL})$, dried $\left(\mathrm{Na}_{2} \mathrm{SO}_{4}\right)$ and concentrated in vacuo. The residue was purified by short FCC (hexanes $100 \%$ EtOAc-MeOH 9:1) to afford the product 11a (134 mg, $98 \%$ ) as a colourless oil; $[\alpha]_{\mathrm{D}}{ }^{20}+13.8$ (c = 0.9, $\left.\mathrm{CHCl}_{3}\right) ; v_{\max } / \mathrm{cm}^{-1}$ (film) 1488 (s), 1454 (m), 1259 (m), 1190 (s), 1041 (m); $\delta_{\mathrm{H}}(400$ $\left.\mathrm{MHz}, \mathrm{CDCl}_{3}\right) 1.93$ (1H, br s, $\underline{\mathrm{H}}$ ), 2.90-3.01 (2H, m, C1- $\left.\underline{\mathrm{H}}_{2} \mathrm{Ph}\right), 3.20-3.27$ (1H, m, C1- $\left.\underline{\mathrm{H}}\right)$, 3.83 (1H, dd, $J=9.0$ and 5.5, C2- $\underline{\mathrm{H}}$ ), 3.87-3.93 (3H, m, C2- $\underline{\mathrm{H}}$ and $\mathrm{NC}_{2} 2 \mathrm{Ph}$ ), 6.69 (1H, dd, $J$ = 9.0 and 5.0, $\operatorname{ArC} \underline{H}), 6.91$ (1H, ddd, $J=9.0,8.0$ and 3.0, $\operatorname{ArC} \underline{H}), 7.15-7.32(11 \mathrm{H}, \mathrm{m}, \operatorname{ArC} \underline{H})$;

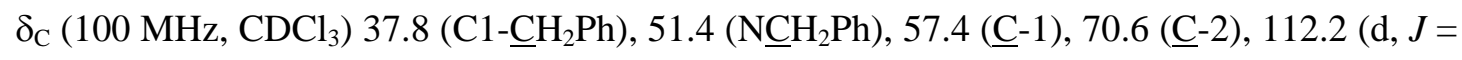
10.0, ArC ), 113.5 (d, $J=8.5$ ), 114.6 (d, $J=22.5$ ), 120.3 (d, $J=26.0$ ), 126.4, 126.9, 128.0, 128.4, 128.5 and 129.3 (ArChH $\times 13), 138.4,140.2,151.6(\mathrm{~d}, J=2.5), 156.6$ (d, $J=243.0)$ $(\mathrm{ArC} \times 4)$; HRMS: $\left(\mathrm{ESI}^{+}\right)$Found: $[\mathrm{M}+\mathrm{H}]^{+} 414.0853, \mathrm{C}_{22} \mathrm{H}_{22} \mathrm{NO}^{79} \mathrm{BrF}$ requires 414.0863.

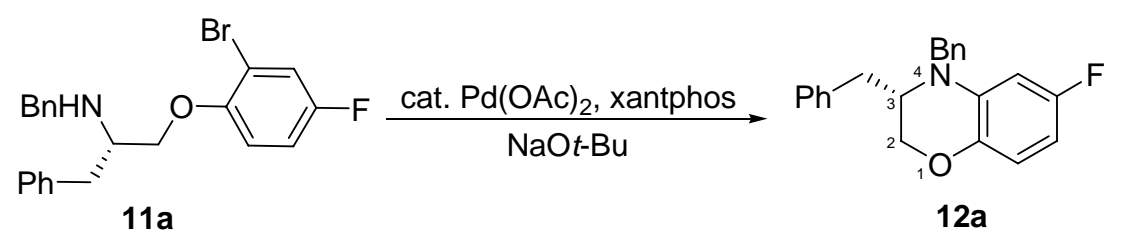

(S)-3,4-Dibenzyl-6-fluoro-3,4-dihydro-2H-benzo[1,4]oxazine (12a): $\mathrm{Pd}(\mathrm{OAc})_{2}$ (2.4 mg, 7 mol \%), xantphos (6.1 mg, $7 \mathrm{~mol} \%$ ), t-BuONa (23 mg, $0.24 \mathrm{mmol}$ ) and amine 11a (67 mg, $0.16 \mathrm{mmol})$ were dissolved in anhydrous $\mathrm{PhMe}(2.2 \mathrm{~mL})$, placed in a re-sealable tube and heated at $100{ }^{\circ} \mathrm{C}$ for $18 \mathrm{~h}$. The mixture was cooled to r.t., diluted with EtOAc (20 mL), washed with water $(10 \mathrm{~mL})$ and then brine $(10 \mathrm{~mL})$, dried $\left(\mathrm{Na}_{2} \mathrm{SO}_{4}\right)$ and concentrated in vacuo. The residue was purified by FCC (EtOAc-hexanes 0:1-2:1) to afford benzoxazine 12a (46 mg, $86 \%$ ) as a pale yellow solid; m.p. $79-81{ }^{\circ} \mathrm{C}$ (EtOAc-hexanes); $[\alpha]_{\mathrm{D}}{ }^{20}-24.0$ (c $=1.5$, $\mathrm{CHCl}_{3}$ ); $v_{\max } / \mathrm{cm}^{-1}$ (film) 1625 (m), 1508 (s), 1215 (m), 1171 (s); $\delta_{\mathrm{H}}\left(400 \mathrm{MHz}, \mathrm{CDCl}_{3}\right) 2.85$ (1H, dd, $J=13.0$ and 9.5, C3-C $\left.\underline{\mathrm{H}}_{2} \mathrm{Ph}\right), 2.96\left(1 \mathrm{H}, \mathrm{dd}, J=13.0\right.$ and 5.5, C3-C $\left.\underline{\mathrm{H}}_{2} \mathrm{Ph}\right), 3.47(1 \mathrm{H}$,

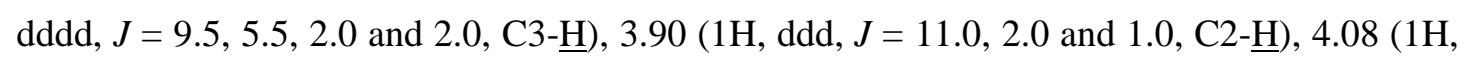
dd, $J=11.0$ and 2.0, C2-프), 4.27 (1H, d, $\left.J=16.5, \mathrm{NC}_{2} \mathrm{Ph}\right), 4.49$ (1H, d, $J=16.5, \mathrm{NC}_{2} \mathrm{Ph}$ ), 


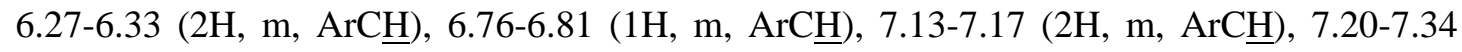

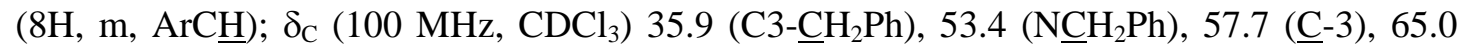

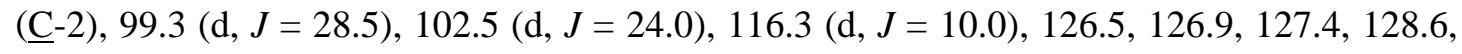
128.8 and $129.5(\mathrm{Ar} \underline{\mathrm{CH}} \times 13), 134.8(\mathrm{~d}, J=11.0), 137.3,138.0,139.4(\mathrm{~d}, J=2.5)$ and 158.4 $(\mathrm{d}, J=236.0)(\operatorname{Ar} \underline{\mathrm{C}} \times 5)$; HRMS: $\left(\mathrm{ESI}^{+}\right)$Found: $[\mathrm{M}+\mathrm{H}]^{+}$334.1603, $\mathrm{C}_{22} \mathrm{H}_{21} \mathrm{NOF}$ requires 334.1602.

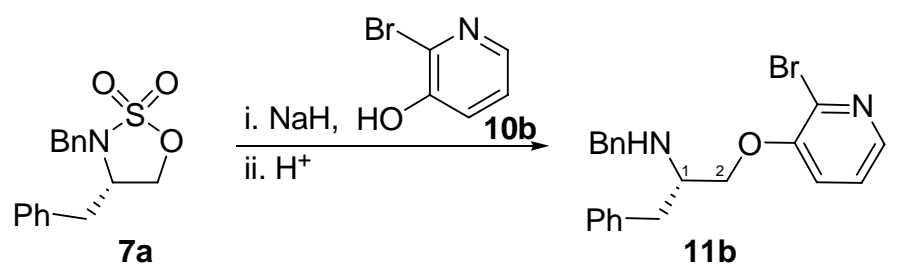

Benzyl[(S)-1-(2-bromopyridin-3-yloxymethyl)-2-phenylethyl]amine (11b): To a solution of 2-bromo-3-hydroxypyridine 10b (115 mg, $0.66 \mathrm{mmol}$ ) in anhydrous DMF (3.8 mL) was added $\mathrm{NaH}$ (60\% dispersion in mineral oil, $26 \mathrm{mg}, 0.66 \mathrm{mmol}$ ) and the mixture was stirred at r.t. for 5 minutes. Cyclic sulfamidate $7 \mathbf{a}(100 \mathrm{mg}, 0.33 \mathrm{mmol})$ was added and the mixture was stirred at r.t. for a further $16 \mathrm{~h}$ prior to the addition of aq. $5 \mathrm{M} \mathrm{HCl}(0.33 \mathrm{~mL})$. The mixture was stirred at r.t. for $2 \mathrm{~h}$, then diluted with aq. $1 \mathrm{M} \mathrm{NaOH}(30 \mathrm{~mL})$ and extracted with $\mathrm{Et}_{2} \mathrm{O}(2$ $\times 20 \mathrm{~mL})$. The organic extracts were combined, washed with aq. $1 \mathrm{M} \mathrm{NaOH}(2 \times 20 \mathrm{~mL})$, dried $\left(\mathrm{Na}_{2} \mathrm{SO}_{4}\right)$ and concentrated in vacuo. The residue was purified by short FCC (hexanes $100 \%$ - EtOAc-MeOH 9:1) to afford the product 11b (124 mg, $98 \%$ ) as a colourless oil; $[\alpha]_{\mathrm{D}}{ }^{20}+10.7\left(\mathrm{c}=1.1, \mathrm{CHCl}_{3}\right) ; v_{\max } / \mathrm{cm}^{-1}$ (film) $2925(\mathrm{~m}), 1562(\mathrm{~m}), 1494(\mathrm{~m}), 1445$ (s), 1416 (s), 1292 (s), 1203 (s), 1128 (m), 1075 (s), 1054 (s); $\delta_{\mathrm{H}}\left(400 \mathrm{MHz}, \mathrm{CDCl}_{3}\right) 1.90$ (1H, br s, $\mathrm{N} \underline{\mathrm{H}}$ ), 2.94 (1H, dd, $J=13.5$ and 7.5, C1- $\left.\underline{\mathrm{H}}_{2} \mathrm{Ph}\right), 3.00$ (1H, dd, $J=13.5$ and 7.5, C1- $\underline{\mathrm{H}}_{2} \mathrm{Ph}$ ), 3.23-3.30 (1H, m, C1- $\underline{\mathrm{H}}), 3.87$ (1H, dd, $J=9.0$ and 4.5, C2- $\underline{\mathrm{H}}$ ), 3.89-3.97 (3H, m, C2- $\underline{\mathrm{H}}$ and $\left.\mathrm{NC}_{2} \mathrm{Ph}\right), 6.97$ (1H, dd, $J=8.5$ and 1.5, $\left.\mathrm{ArC} \underline{\mathrm{H}}\right), 7.12-7.30$ (11H, m, $\left.\operatorname{ArC} \underline{H}\right), 7.96$ (1H, dd, $J=$ 4.5 and 1.5, $\mathrm{ArC} \underline{\mathrm{H}}) ; \delta_{\mathrm{C}}\left(100 \mathrm{MHz}, \mathrm{CDCl}_{3}\right) 37.7\left(\mathrm{C} 1-\underline{\mathrm{CH}}_{2} \mathrm{Ph}\right), 51.4\left(\mathrm{NCH}_{2} \mathrm{Ph}\right), 57.1(\underline{\mathrm{C}}-1)$, 70.2 (C-2), 119.5, 123.3, 126.4, 127.0, 128.0, 128.4, 128.5 and $129.2(\mathrm{Ar} \underline{\mathrm{CH}} \times 12), 133.0$, 138.2 and $140.1(\mathrm{Ar} \underline{\mathrm{C}} \times 3), 141.3(\mathrm{Ar} \underline{\mathrm{CH}}), 152.0$ (ArC $)$; HRMS: $\left(\mathrm{ESI}^{+}\right)$Found: $[\mathrm{M}+\mathrm{H}]^{+}$ 397.0833, $\mathrm{C}_{21} \mathrm{H}_{22} \mathrm{~N}_{2} \mathrm{O}^{79} \mathrm{Br}$ requires 397.0837.

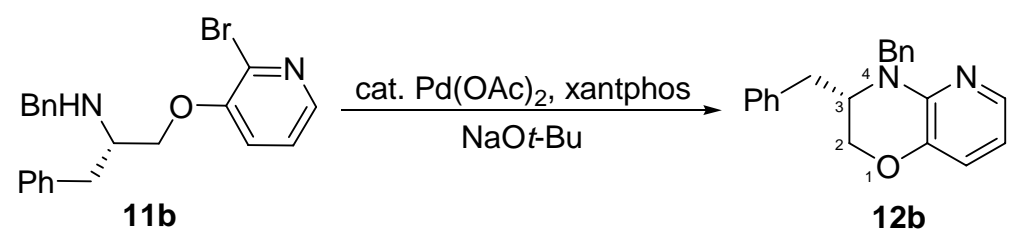


(S)-3,4-Dibenzyl-3,4-dihydro-2H-pyrido[3,2-b][1,4]oxazine (12b): $\mathrm{Pd}(\mathrm{OAc})_{2}(2.4 \mathrm{mg}, 7$ mol \%), xantphos (6.1 mg, $7 \mathrm{~mol} \%), t$-BuONa (24 mg, $0.23 \mathrm{mmol}$ ) and amine 11b (70 mg, $0.18 \mathrm{mmol})$ were dissolved in anhydrous $\mathrm{PhMe}(2.2 \mathrm{~mL})$, placed in a re-sealable tube and heated at $100{ }^{\circ} \mathrm{C}$ for $21.5 \mathrm{~h}$. The mixture was cooled to r.t., diluted with EtOAc (20 mL), washed with water $(10 \mathrm{~mL})$ and then brine $(10 \mathrm{~mL})$, dried $\left(\mathrm{Na}_{2} \mathrm{SO}_{4}\right)$ and concentrated in vacuo. The residue was purified by FCC (EtOAc-hexanes 0:1-1:1) to afford benzoxazine 12b (45 mg, $79 \%$ ) as a colourless solid; m.p. $79-81{ }^{\circ} \mathrm{C}$ (EtOAc-hexanes); $[\alpha]_{\mathrm{D}}{ }^{20}-74.1$ (c $=0.3$, $\mathrm{CHCl}_{3}$ ); $v_{\max } / \mathrm{cm}^{-1}$ (film) 3026 (m), 1602 (m), 1489 (s), 1453 (m), 1356 (m), 1209 (s); $\delta_{\mathrm{H}}$ (400 MHz, $\left.\mathrm{CDCl}_{3}\right) 2.70$ (1H, dd, $J=13.5$ and 10.0, C3-C $\left.\underline{H}_{2} \mathrm{Ph}\right), 2.90$ (1H, dd, $J=13.5$ and 5.0, C3- $\underline{\mathrm{C}}_{2} \mathrm{Ph}$ ), 3.41 (1H, dddd, $J=10.0,5.0,2.5$ and 1.5, C3- $\underline{\mathrm{H}}$ ), 3.67 (1H, ddd, $J=11.0$, 2.5 and 1.5, C2-파), 3.92 (1H, dd, $J=11.0$ and 1.5, C2- $\underline{\mathrm{H}}), 4.06\left(1 \mathrm{H}, \mathrm{d}, J=15.5, \mathrm{NC}_{2} \mathrm{Ph}\right)$, $5.54\left(1 \mathrm{H}, \mathrm{d}, J=15.5, \mathrm{NC}_{2} \mathrm{Ph}\right), 6.48$ (1H, dd, $J=7.5$ and 5.0, ArC$), 6.94$ (1H, dd, $J=7.5$ and 1.5, $\operatorname{ArC} \underline{H}), 7.02-7.06$ (2H, m, ArC $\underline{H}), 7.11-7.26(8 \mathrm{H}, \mathrm{m}, \operatorname{ArC} \underline{\mathrm{H}}), 7.73(1 \mathrm{H}, \mathrm{dd}, J=5.0$ and 1.5, $\mathrm{ArC} \underline{\mathrm{H}}) ; \delta_{\mathrm{C}}\left(100 \mathrm{MHz}, \mathrm{CDCl}_{3}\right) 35.6\left(\mathrm{C} 3-\underline{\mathrm{CH}}_{2} \mathrm{Ph}\right), 48.3\left(\mathrm{~N}_{\underline{C}} \mathrm{H}_{2} \mathrm{Ph}\right), 55.3(\underline{\mathrm{C}}-3), 64.8$ (ㄷ-2), 112.7, 121.3, 126.5, 127.2, 128.0, 128.5, 128.6 and 129.4 ( $\mathrm{Ar} \underline{\mathrm{CH}} \times 12), 137.9,138.2$

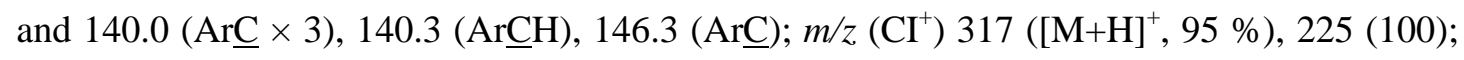
HRMS: (CI ${ }^{+}$) Found: $[\mathrm{M}+\mathrm{H}]^{+} 317.1640, \mathrm{C}_{21} \mathrm{H}_{21} \mathrm{~N}_{2} \mathrm{O}$ requires 317.1654

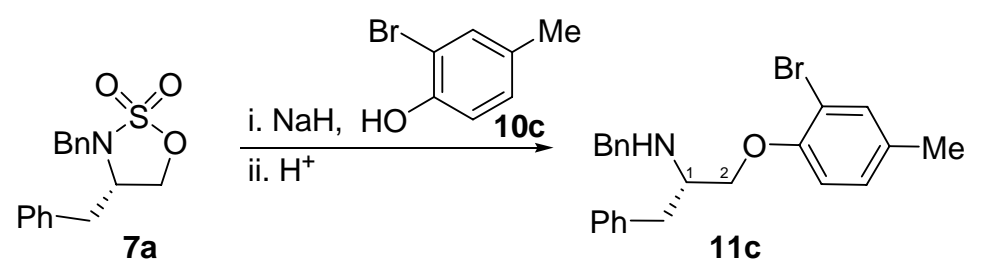

Benzyl[(S)-1-benzyl-2-(2-bromo-4-methylphenoxy)ethyl]amine (11c): To a solution of 2bromo-4-methylphenol 10c (123 mg, $0.66 \mathrm{mmol}$ ) in anhydrous DMF (3.8 mL) was added $\mathrm{NaH}$ (60 \% dispersion in mineral oil, $26 \mathrm{mg}, 0.66 \mathrm{mmol}$ ) and the mixture was stirred at r.t. for 5 minutes. Cyclic sulfamidate $7 \mathbf{a}(100 \mathrm{mg}, 0.33 \mathrm{mmol})$ was added and the mixture was stirred at r.t. for a further $22 \mathrm{~h}$ prior to the addition of aq. $5 \mathrm{M} \mathrm{HCl}(0.33 \mathrm{~mL})$. The mixture was stirred at r.t. for $3 \mathrm{~h}$, then diluted with aq. $1 \mathrm{M} \mathrm{NaOH}(30 \mathrm{~mL})$ and extracted with $\mathrm{Et}_{2} \mathrm{O}(2 \times$ $20 \mathrm{~mL})$. The organic extracts were combined, washed with aq. $1 \mathrm{M} \mathrm{NaOH}(2 \times 20 \mathrm{~mL})$, dried $\left(\mathrm{Na}_{2} \mathrm{SO}_{4}\right)$ and concentrated in vacuo. The residue was purified by short FCC (hexanes $100 \%$ EtOAc-MeOH 9:1) to afford the product 11c (138 mg, $99 \%)$ as a colourless oil; $[\alpha]_{\mathrm{D}}{ }^{20}+23.2$ (c = 0.7, $\left.\mathrm{CHCl}_{3}\right) ; v_{\max } / \mathrm{cm}^{-1}$ (film) 2923 (m), 1495 (s), 1454 (m), 1286 (m), 1253 (s), 1050

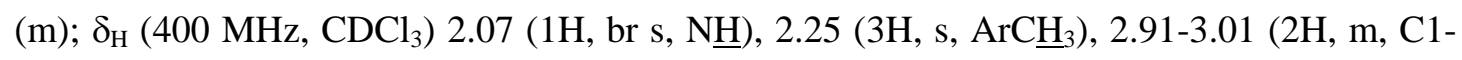
$\left.\mathrm{C}_{2} \mathrm{Ph}\right), 3.20-3.28$ (1H, m, C1- $\left.\underline{\mathrm{H}}\right), 3.85$ (1H, dd, $J=9.5$ and 5.5, C2- $\left.\underline{\mathrm{H}}\right), 3.88-3.93$ (3H, m, C2- $\underline{\mathrm{H}}$ and $\left.\mathrm{NC}_{2} \mathrm{Ph}\right), 6.66$ (1H, d, $\left.J=8.5, \mathrm{ArC} \underline{\mathrm{H}}\right), 6.97$ (1H, dd, $J=8.5$ and 2.0, ArC$), 7.16-$ 
$7.31(10 \mathrm{H}, \mathrm{m}, \mathrm{ArC} \underline{\mathrm{H}}), 7.35$ (1H, d, $J=2.0, \mathrm{ArC} \underline{\mathrm{H}}) ; \delta_{\mathrm{C}}\left(100 \mathrm{MHz}, \mathrm{CDCl}_{3}\right) 20.1\left(\mathrm{Ar}^{\mathrm{C}} \mathrm{H}_{3}\right), 37.8$ (C1- $\underline{\mathrm{CH}}_{2} \mathrm{Ph}$ ), 51.4 ( $\mathrm{NCH}_{2} \mathrm{Ph}$ ), 57.4 (ㄷ-1), 70.0 (드-2), 111.9 (ArC), 113.0, 126.3, 126.9, 128.0, 128.4 (2 signals), 128.8 and $129.3(\mathrm{Ar} \underline{\mathrm{C}} \mathrm{H} \times 12), 131.6(\mathrm{Ar} \underline{\mathrm{C}}), 133.6(\mathrm{Ar} \underline{\mathrm{CH}}), 138.6,140.2$ and 152.9 (ArC $\times 3$ ); HRMS: $\left(\mathrm{ESI}^{+}\right)$Found: $[\mathrm{M}+\mathrm{H}]^{+} 410.1105, \mathrm{C}_{23} \mathrm{H}_{25} \mathrm{NO}^{79} \mathrm{Br}$ requires 410.1114 .

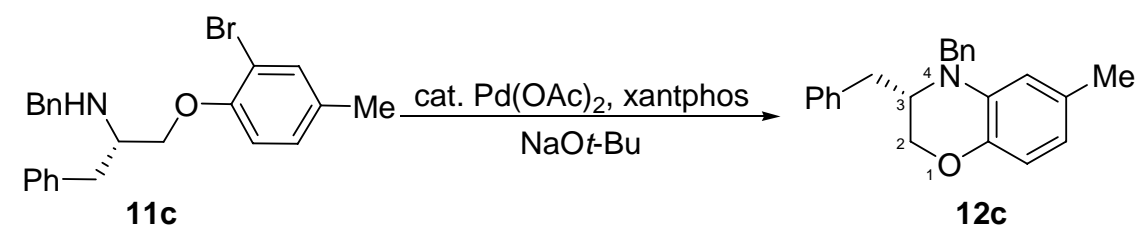

(S)-3,4-Dibenzyl-6-methyl-3,4-dihydro-2H-benzo[1,4]oxazine (12c): $\mathrm{Pd}(\mathrm{OAc})_{2}$ (2.5 mg, 7 mol \%), xantphos (6.2 mg, $7 \mathrm{~mol} \%), t$-BuONa (25 mg, $0.25 \mathrm{mmol}$ ) and amine 11c (71 mg, $0.17 \mathrm{mmol})$ were dissolved in anhydrous PhMe $(2.3 \mathrm{~mL})$, placed in a re-sealable tube and heated at $100{ }^{\circ} \mathrm{C}$ for $24 \mathrm{~h}$. The mixture was cooled to r.t., diluted with EtOAc (20 mL), washed with water $(10 \mathrm{~mL})$ and then brine $(10 \mathrm{~mL})$, dried $\left(\mathrm{Na}_{2} \mathrm{SO}_{4}\right)$ and concentrated in vacuo. The residue was purified by FCC (EtOAc-hexanes 0:1-1:4) to afford benzoxazine 12c (40 mg, $72 \%$ ) as a colourless oil; $[\alpha]_{\mathrm{D}}{ }^{20}-16.4\left(\mathrm{c}=0.7, \mathrm{CHCl}_{3}\right) ; v_{\max } / \mathrm{cm}^{-1}$ (film) $1611(\mathrm{~m})$, 1511 (s), 1453 (m), 1267 (m), 1217 (s); $\delta_{\mathrm{H}}\left(400 \mathrm{MHz}, \mathrm{CDCl}_{3}\right) 2.20$ (3H, s, $\left.\mathrm{ArC}_{3}\right), 2.84(1 \mathrm{H}$, dd, $J=13.0$ and 9.5, C3-C $\left.\underline{H}_{2} \mathrm{Ph}\right), 2.92$ (1H, dd, $J=13.0$ and 5.5, C3- $\underline{\mathrm{H}}_{2} \mathrm{Ph}$ ), 3.41 (1H, dddd, $J=9.5,5.5,2.0$ and 2.0, C3- $\underline{\mathrm{H}}), 3.91$ (1H, ddd, $J=10.5,2.0$ and 1.0, C2- $\underline{\mathrm{H}}$ ), 4.04 (1H, dd, $J=$ 10.5 and 2.0, C2- $\underline{\mathrm{H}}), 4.29$ (1H, d, $\left.J=16.0, \mathrm{NC}_{2} \mathrm{Ph}\right), 4.56$ (1H, d, $\left.J=16.0, \mathrm{NC}_{2} \mathrm{Ph}\right), 6.44-$

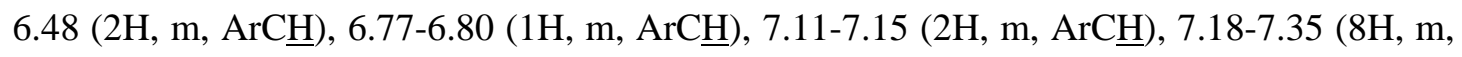
$\operatorname{ArC} \underline{H}) ; \delta_{\mathrm{C}}\left(100 \mathrm{MHz}, \mathrm{CDCl}_{3}\right) 21.2\left(\mathrm{Ar}_{\underline{C}}{ }_{3}\right), 35.6\left(\mathrm{C}^{-} \underline{\mathrm{CH}}_{2} \mathrm{Ph}\right), 53.5\left(\mathrm{~N}^{2} H_{2} \mathrm{Ph}\right), 57.6(\underline{\mathrm{C}}-3)$, 64.9 (ㄷ-2), 113.2, 116.0, 117.7, 126.3, 127.1 (2 signals), 128.5 (2 signals) and 129.5 (ArㅁH $\times$ 13), 131.2, 133.8, 138.2, 138.4 and $141.4\left(\mathrm{ArC} \times\right.$ 5); HRMS: $\left(\mathrm{ESI}^{+}\right)$Found: $[\mathrm{M}+\mathrm{H}]^{+}$ $330.1854, \mathrm{C}_{23} \mathrm{H}_{24} \mathrm{NO}$ requires 330.1852 .

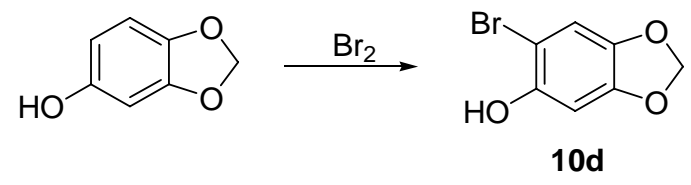

6-Bromobenzo[1,3]dioxol-5-ol (10d): ${ }^{2}$ To a cooled $\left(0{ }^{\circ} \mathrm{C}\right)$ solution of sesamol $(2.00 \mathrm{~g}, 14.5$ $\mathrm{mmol}$ ) in $\mathrm{AcOH}(4.4 \mathrm{~mL})$ was added, dropwise over 5 minutes, a solution of bromine (584 $\mu \mathrm{L}, 11.40 \mathrm{mmol})$ in $\mathrm{AcOH}(2.50 \mathrm{~mL})$. The mixture was then immediately poured onto ice and the resulting green solid was collected by filtration, washed copiously with water (ca. 100 $\mathrm{mL}$ ) and dried (60 ${ }^{\circ} \mathrm{C}, 15 \mathrm{mmHg}, 12 \mathrm{~h}$ ) to afford the title compound $10 \mathrm{~d}$ (1.84 g, $59 \%$ ) as a dull green solid; m.p. 86-87 ${ }^{\circ} \mathrm{C}$ ( $\mathrm{Et}_{2} \mathrm{O}$-hexanes) [Lit., ${ }^{2}$ 87-88 ${ }^{\circ} \mathrm{C}$ (no recrystallisation solvent 
quoted)]; $\delta_{\mathrm{H}}\left(270 \mathrm{MHz}, \mathrm{CDCl}_{3}\right) 5.21(1 \mathrm{H}, \mathrm{br} \mathrm{s}, \mathrm{ArO} \underline{\mathrm{H}}), 5.91\left(2 \mathrm{H}, \mathrm{s}, \mathrm{OC}_{2} \mathrm{O}\right), 6.59(1 \mathrm{H}, \mathrm{s}$, $\operatorname{ArC} \underline{H}), 6.89(1 \mathrm{H}, \mathrm{s}, \mathrm{ArC} \underline{\mathrm{H}})$. The spectroscopic properties of this compound were consistent with the data available in the literature. ${ }^{2}$

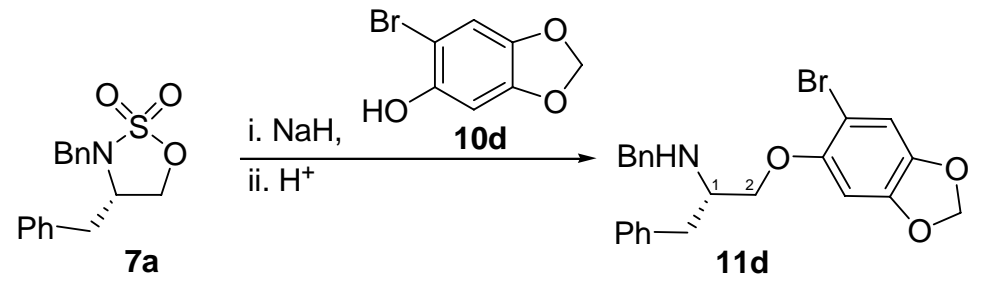

Benzyl[(S)-1-benzyl-2-(6-bromobenzo[1,3]dioxol-5-yloxy)ethyl]amine (11d): To a solution of 6-bromobenzo[1,3]dioxol-5-ol 10d (72 $\mathrm{mg}, 0.33 \mathrm{mmol}$ ) in anhydrous DMF (1.9 mL) was added $\mathrm{NaH}$ (60 \% dispersion in mineral oil, $13 \mathrm{mg}, 0.33 \mathrm{mmol}$ ) and the mixture was stirred at r.t. for 5 minutes. Cyclic sulfamidate $7 \mathbf{a}$ (50 $\mathrm{mg}, 0.17 \mathrm{mmol}$ ) was added and the mixture was stirred at r.t. for a further $16 \mathrm{~h}$ prior to the addition of aq. $5 \mathrm{M} \mathrm{HCl}(0.17 \mathrm{~mL})$. The mixture was stirred at r.t. for $2 \mathrm{~h}$, then diluted with aq. $1 \mathrm{M} \mathrm{NaOH}(30 \mathrm{~mL})$ and extracted with $\mathrm{Et}_{2} \mathrm{O}(2$ $\times 20 \mathrm{~mL})$. The organic extracts were combined, washed with aq. $1 \mathrm{M} \mathrm{NaOH}(2 \times 20 \mathrm{~mL})$, dried $\left(\mathrm{Na}_{2} \mathrm{SO}_{4}\right)$ and concentrated in vacuo. The residue was purified by short FCC (hexanes $100 \%$ - EtOAc-MeOH 9:1) to afford the product 11d (60 mg, $83 \%$ ) as a colourless oil; $[\alpha]_{\mathrm{D}}^{20}+38.7\left(\mathrm{c}=0.3, \mathrm{CHCl}_{3}\right) ; v_{\max } / \mathrm{cm}^{-1}(\mathrm{film}) 1501(\mathrm{~m}), 1474(\mathrm{~s}), 1183(\mathrm{~s}), 1117(\mathrm{~m}), 1037$ (s); $\delta_{\mathrm{H}}\left(400 \mathrm{MHz}, \mathrm{CDCl}_{3}\right) 2.34(1 \mathrm{H}$, br s, $\underline{\mathrm{H}}), 2.92\left(1 \mathrm{H}, \mathrm{dd}, J=13.5\right.$ and 7.5, C1-C $\left.\underline{\mathrm{H}}_{2} \mathrm{Ph}\right)$, 2.98 (1H, dd, $J=13.5$ and 6.5, C1- $\underline{\mathrm{H}}_{2} \mathrm{Ph}$ ), 3.18-3.25 (1H, m, C1- $\underline{\mathrm{H}}$ ), 3.79 (1H, dd, $J=9.0$

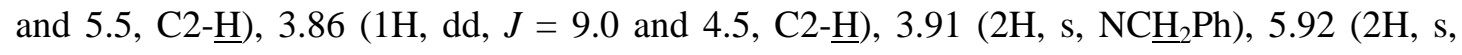
OC $\left.\underline{H}_{2} \mathrm{O}\right), 6.43(1 \mathrm{H}, \mathrm{s}, \operatorname{ArC} \underline{\mathrm{H}}), 6.99(1 \mathrm{H}, \mathrm{s}, \operatorname{ArC} \underline{\mathrm{H}}), 7.16-7.33(10 \mathrm{H}, \mathrm{m}, \operatorname{ArC} \underline{\mathrm{H}}) ; \delta_{\mathrm{C}}(100 \mathrm{MHz}$,

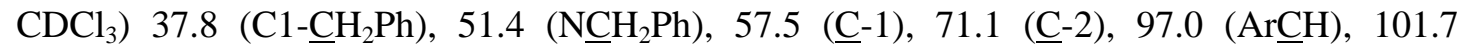
$\left(\mathrm{O}_{\underline{C}} \mathrm{H}_{2} \mathrm{O}\right), 102.1(\mathrm{Ar} \underline{\mathrm{C}}), 112.5,126.3,126.9,128.1,128.4,128.5$ and $129.3(\mathrm{Ar} \underline{\mathrm{CH}} \times 11)$, 138.5, 140.1, 142.0, 147.6 and $150.2(\operatorname{Ar} \underline{C} \times 5)$; HRMS: $\left(\mathrm{ESI}^{+}\right)$Found: $[\mathrm{M}+\mathrm{H}]^{+}$440.0856, $\mathrm{C}_{23} \mathrm{H}_{23} \mathrm{NO}_{3}{ }^{79} \mathrm{Br}$ requires 440.0856 .

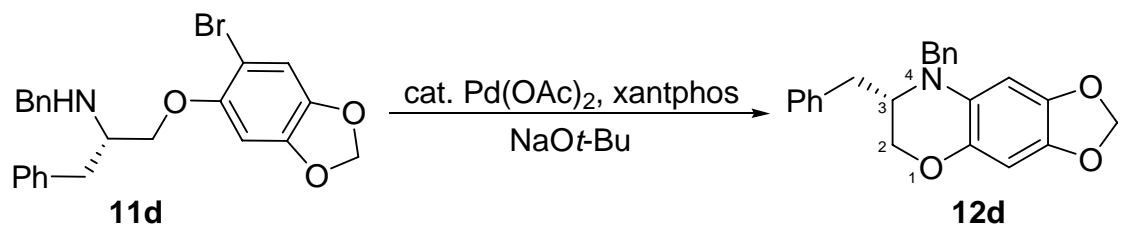

(S)-7,8-Dibenzyl-7,8-dihydro-6H-[1,3]dioxolo[4',5':4,5]benzo[1,2-b][1,4]oxazine (12d): $\mathrm{Pd}(\mathrm{OAc})_{2}$ (1.5 mg, $7 \mathrm{~mol} \%$ ), xantphos (3.8 mg, $7 \mathrm{~mol} \%$ ), $t$-BuONa (15 mg, $0.15 \mathrm{mmol}$ ) and amine 11d (45 mg, $0.10 \mathrm{mmol}$ ) were dissolved in anhydrous PhMe (1.4 mL), placed in a resealable tube and heated at $100{ }^{\circ} \mathrm{C}$ for $18 \mathrm{~h}$. The mixture was cooled to r.t., diluted with 
EtOAc $(20 \mathrm{~mL})$, washed with water $(10 \mathrm{~mL})$ and then brine $(10 \mathrm{~mL})$, dried $\left(\mathrm{Na}_{2} \mathrm{SO}_{4}\right)$ and concentrated in vacuo. The residue was purified by FCC (EtOAc-hexanes 1:1) to afford benzoxazine 12d (18 mg, $50 \%$ ) as a colourless solid. Continued elution afforded recovered amine 11d (4 mg, $9 \%$ ) as a colourless oil.

Data for 12d: m.p. $102-103{ }^{\circ} \mathrm{C}\left(\mathrm{Et}_{2} \mathrm{O}-\right.$ hexanes$) ;[\alpha]_{\mathrm{D}}{ }^{20}+21.9$ (c $\left.=0.7, \mathrm{CHCl}_{3}\right) ; v_{\max } / \mathrm{cm}^{-1}$ (film) 1505 (s), 1481 (s), 1164 (s), 1038 (s); $\delta_{\mathrm{H}}\left(400 \mathrm{MHz}, \mathrm{CDCl}_{3}\right) 2.78$ (1H, dd, $J=13.0$ and 9.5, C3- $\underline{\mathrm{CH}}_{2} \mathrm{Ph}$ ), 2.87 (1H, dd, $J=13.0$ and 6.0, C3-C $\underline{H}_{2} \mathrm{Ph}$ ), 3.35 (1H, dddd, $J=9.5,6.0,2.0$ and 2.0, C3- $\underline{\mathrm{H}}$ ), 3.87 (1H, ddd, $J=10.5,2.0$ and 1.0, C2- $\underline{\mathrm{H}}$ ), 4.02 (1H, dd, $J=10.5$ and 2.0, C2- $\underline{\mathrm{H}}$ ), 4.26 (1H, d, $\left.J=16.0, \mathrm{NC}_{2} \mathrm{Ph}\right), 4.33$ (1H, d, $\left.J=16.0, \mathrm{NC}_{2} \mathrm{Ph}\right), 5.80$ (1H, d, $J=1.0$, $\left.\mathrm{OC}_{2} \mathrm{O}\right), 5.81\left(1 \mathrm{H}, \mathrm{d}, J=1.0, \mathrm{OC}_{2} \mathrm{O}\right), 6.29(1 \mathrm{H}, \mathrm{s}, \operatorname{ArC} \underline{\mathrm{H}}), 6.51(1 \mathrm{H}, \mathrm{s}, \operatorname{ArC} \underline{\mathrm{H}}), 7.09-7.13$

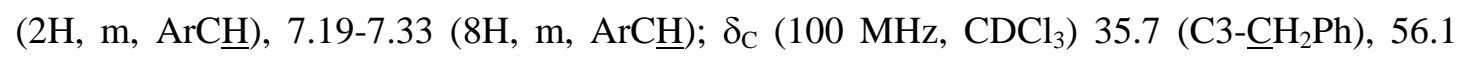

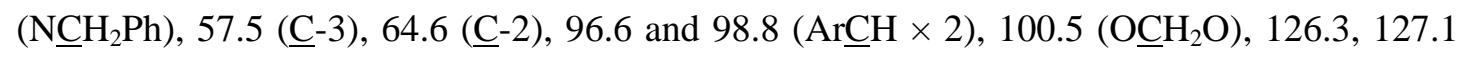
and 127.2 $(\mathrm{Ar} \underline{\mathrm{CH}} \times$ 4), $127.8(\mathrm{Ar} \underline{\mathrm{C}}), 128.5,128.6$ and $129.5(\mathrm{Ar} \underline{\mathrm{CH}} \times 6), 137.9,138.2,138.5$, 139.1 and $142.0\left(\mathrm{Ar} \underline{\mathrm{C}} \times 5\right.$ ); HRMS: $\left(\mathrm{ESI}^{+}\right)$Found: $[\mathrm{M}+\mathrm{H}]^{+}$359.1514, $\mathrm{C}_{23} \mathrm{H}_{22} \mathrm{NO}_{3}$ requires 359.1516.

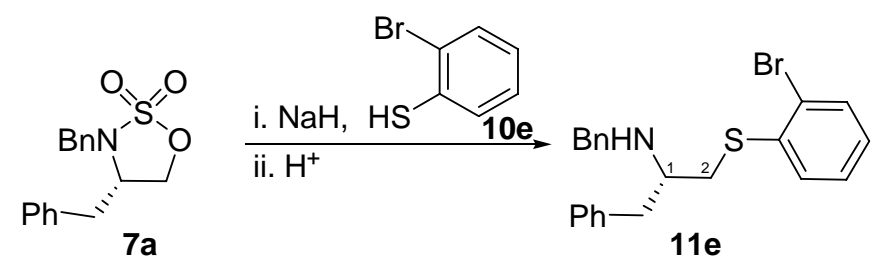

Benzyl[(S)-1-benzyl-2-(2-bromophenylsulfanyl)ethyl]amine (11e): To a solution of 2bromothiophenol 10e (125 mg, $0.66 \mathrm{mmol})$ in anhydrous DMF (3.8 mL) was added $\mathrm{NaH}$ (60 \% dispersion in mineral oil, $26 \mathrm{mg}, 0.33 \mathrm{mmol}$ ) and the mixture was stirred at r.t. for 5 minutes. Cyclic sulfamidate $7 \mathbf{a}(100 \mathrm{mg}, 0.33 \mathrm{mmol})$ was added and the mixture was stirred at r.t. for a further $19 \mathrm{~h}$ prior to the addition of aq. $5 \mathrm{M} \mathrm{HCl}(0.33 \mathrm{~mL})$. The mixture was stirred at r.t. for $3 \mathrm{~h}$, then diluted with aq. $1 \mathrm{M} \mathrm{NaOH}(30 \mathrm{~mL})$ and extracted with $\mathrm{Et}_{2} \mathrm{O}(3 \times$ $20 \mathrm{~mL})$. The organic extracts were combined, washed with aq. $1 \mathrm{M} \mathrm{NaOH}(3 \times 20 \mathrm{~mL})$, dried $\left(\mathrm{Na}_{2} \mathrm{SO}_{4}\right)$ and concentrated in vacuo. The residue was purified by short FCC (hexanes $100 \%$ EtOAc-MeOH 9:1) to afford the product 11e (136 mg, $100 \%$ ) as a colourless, crystalline solid; m.p. 118-120 ${ }^{\circ} \mathrm{C}\left(\mathrm{Et}_{2} \mathrm{O}-\right.$ hexanes); $[\alpha]_{\mathrm{D}}{ }^{20}+44.4$ (c = 0.9, $\mathrm{CHCl}_{3}$ ); $v_{\max } / \mathrm{cm}^{-1}$ (film) 2923 (m), 1494 (m), 1449 (s), 1108 (m), 1020 (m); $\delta_{\mathrm{H}}\left(400 \mathrm{MHz}, \mathrm{CDCl}_{3}\right) 1.76(1 \mathrm{H}, \mathrm{br}$ s, N $\underline{\mathrm{H}}$ ), 2.85-3.08 (5H, m, C2- $\underline{\mathrm{H}}, \mathrm{C} 1-\underline{\mathrm{H}}$ and $\left.\mathrm{C} 1-\mathrm{C}_{2} \mathrm{Ph}\right), 3.81\left(2 \mathrm{H}, \mathrm{s}, \mathrm{NC}_{2} \mathrm{Ph}\right), 6.96-7.03(2 \mathrm{H}, \mathrm{m}$, $\operatorname{ArC} \underline{H}), 7.11-7.31(11 \mathrm{H}, \mathrm{m}, \mathrm{ArC} \underline{\mathrm{H}}), 7.51-7.54(1 \mathrm{H}, \mathrm{m}, \mathrm{ArC} \underline{\mathrm{H}}) ; \delta_{\mathrm{C}}\left(100 \mathrm{MHz}, \mathrm{CDCl}_{3}\right) 37.2$

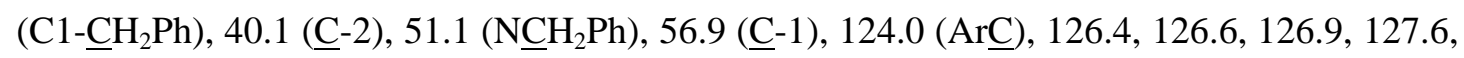
128.0, 128.3, 128.5, 126.9, 129.4 and $132.9(\mathrm{Ar} \underline{\mathrm{CH}} \times 14), 137.7,138.4$ and $139.9(\operatorname{Ar} \underline{\mathrm{C}} \times 3)$; 
m/z $\left(\mathrm{CI}^{+}\right) 412$ and $414\left([\mathrm{M}+\mathrm{H}]^{+}, 50\right.$ and $\left.48 \%\right), 210$ (100); HRMS: $\left(\mathrm{CI}^{+}\right)$Found: $[\mathrm{M}+\mathrm{H}]^{+}$ 412.0743, $\mathrm{C}_{22} \mathrm{H}_{23} \mathrm{NS}^{79} \mathrm{Br}$ requires 412.0735 .

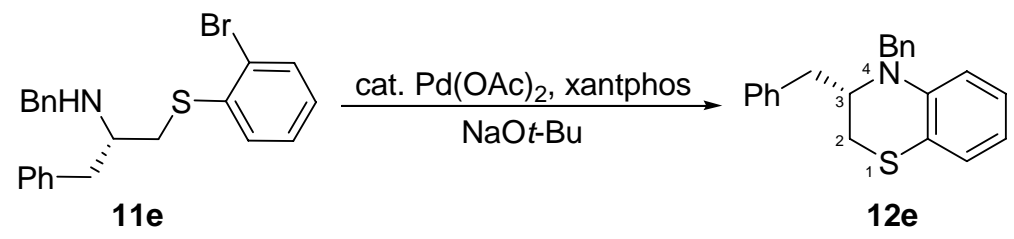

(S)-3,4-Dibenzyl-3,4-dihydro-2H-benzo[1,4]thiazine (12e): $\mathrm{Pd}(\mathrm{OAc})_{2}$ (2.4 mg, $6 \mathrm{~mol} \%$ ), xantphos (6.1 mg, $6 \mathrm{~mol} \%$ ), $t$-BuONa (24 mg, $0.23 \mathrm{mmol}$ ) and amine 11e (71 mg, 0.17 mmol) were dissolved in anhydrous PhMe $(2.2 \mathrm{~mL})$, placed in a re-sealable tube and heated at $100{ }^{\circ} \mathrm{C}$ for 24 h. The mixture was cooled to r.t., diluted with EtOAc $(20 \mathrm{~mL})$, washed with water $(10 \mathrm{~mL})$ and then brine $(10 \mathrm{~mL})$, dried $\left(\mathrm{Na}_{2} \mathrm{SO}_{4}\right)$ and concentrated in vacuo. The residue was purified by FCC (EtOAc-hexanes 1:1) to afford benzoxazine 12e (50 mg, $89 \%$ ) as a cream solid; m.p. $108-111{ }^{\circ} \mathrm{C}\left(\mathrm{Et}_{2} \mathrm{O}\right.$-hexanes); $[\alpha]_{\mathrm{D}}{ }^{20}+14.1$ (c $\left.=0.9, \mathrm{CHCl}_{3}\right) ; v_{\max } / \mathrm{cm}^{-1}$ (film) 1587 (m), 1486 (s), 1452 (m), 1439 (m), 1241 (m); $\delta_{\mathrm{H}}\left(400 \mathrm{MHz}, \mathrm{CDCl}_{3}\right) 2.67$ (1H, dd, $J=12.5$ and 2.5, C2- $\underline{\mathrm{H}}), 2.99-3.14$ (3H, m, C2- $\underline{\mathrm{H}}$ and $\left.\mathrm{C} 3-\mathrm{C}_{2} \mathrm{Ph}\right), 3.77-3.83$ (1H, m, C3- $\underline{\mathrm{H}}$ ), 4.38 (1H, d, $\left.J=16.5, \mathrm{NC}_{2} \mathrm{Ph}\right), 4.58$ (1H, d, $\left.J=16.5, \mathrm{NC}_{2} \mathrm{Ph}\right), 6.57-6.66$ (2H, m, ArC$)$ ),

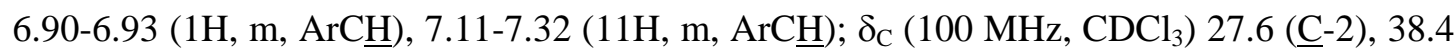
(C3- $\underline{\mathrm{CH}}_{2} \mathrm{Ph}$ ), 55.3 ( $\mathrm{NCH}_{2} \mathrm{Ph}$ ), 58.4 (ㅡ-3), 117.0 (ArC), 113.9, 117.2, 125.8, 126.4, 126.7, 127.0, 127.6, 128.5, 128.7 and $129.4(\operatorname{Ar} \underline{\mathrm{CH}} \times 14), 138.1,138.5$ and $141.9(\operatorname{ArC} \times 3) ; m / z$ $\left(\mathrm{CI}^{+}\right) 332\left([\mathrm{M}+\mathrm{H}]^{+}, 100 \%\right)$; HRMS: $\left(\mathrm{CI}^{+}\right)$Found: $[\mathrm{M}+\mathrm{H}]^{+}$332.1468, $\mathrm{C}_{22} \mathrm{H}_{22} \mathrm{NS}$ requires 332.1473 .

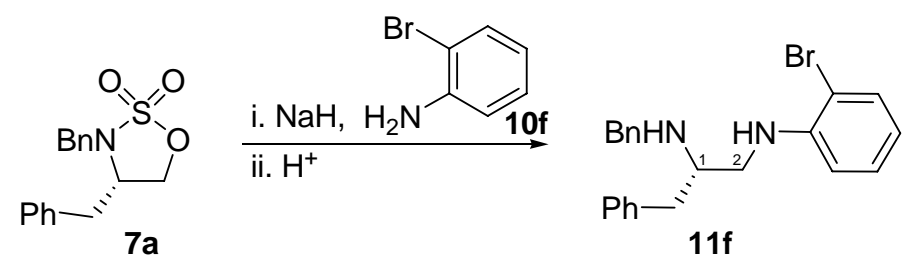

(S)- $N^{2}$-Benzyl- $N^{1}$-(2-bromophenyl)-3-phenylpropane-1,2-diamine (11f): To a solution of 2-bromoaniline $10 f(133 \mathrm{mg}, 0.66 \mathrm{mmol})$ in anhydrous DMF (3.8 mL) was added NaH (60\% dispersion in mineral oil, $26 \mathrm{mg}, 0.33 \mathrm{mmol}$ ) and the mixture was stirred at r.t. for 15 minutes. Cyclic sulfamidate $\mathbf{7 a}$ (100 mg, $0.33 \mathrm{mmol}$ ) was added and the mixture was stirred at r.t. for a further $16 \mathrm{~h}$ prior to the addition of aq. $5 \mathrm{M} \mathrm{HCl}(0.33 \mathrm{~mL})$. The mixture was stirred at r.t. for $2 \mathrm{~h}$, then diluted with aq. $1 \mathrm{M} \mathrm{NaOH}(30 \mathrm{~mL})$ and extracted with $\mathrm{CH}_{2} \mathrm{Cl}_{2}(3 \times 20 \mathrm{~mL})$. The organic extracts were combined, dried $\left(\mathrm{Na}_{2} \mathrm{SO}_{4}\right)$ and concentrated in vacuo. The residue was purified by FCC (hexanes-EtOAc 4:1) to afford the product $\mathbf{1 1 f}$ (73 $\mathrm{mg}, 56 \%$ ) as a colourless 
oil; $[\alpha]_{\mathrm{D}}^{20}+80.0$ (c = 0.2, $\mathrm{CHCl}_{3}$ ); $v_{\max } / \mathrm{cm}^{-1}$ (film) 3351 (br m), 1585 (s), 1495 (s), 1453 (m), $1018(\mathrm{~m}) ; \delta_{\mathrm{H}}\left(400 \mathrm{MHz}, \mathrm{CDCl}_{3}\right) 2.82\left(1 \mathrm{H}, \mathrm{dd}, J=13.5\right.$ and 6.5, C1- $\left.\underline{\mathrm{H}}_{2} \mathrm{Ph}\right), 2.89-3.02(2 \mathrm{H}$, m, C1- $\underline{\mathrm{C}}_{2} \mathrm{Ph}$ and $\left.\mathrm{C} 2-\underline{\mathrm{H}}\right), 3.12-3.21(2 \mathrm{H}, \mathrm{m}, \mathrm{C} 2-\underline{\mathrm{H}}$ and $\mathrm{C} 1-\underline{\mathrm{H}}), 3.78(1 \mathrm{H}, \mathrm{d}, J=13.0$, $\mathrm{NC}_{2} \mathrm{Ph}$ ), $3.83\left(1 \mathrm{H}, \mathrm{d}, J=13.0, \mathrm{NC}_{2} \mathrm{Ph}\right), 5.06-5.13(1 \mathrm{H}$, br m, N$), 6.49$ (1H, dd, $J=8.0$ and 1.0, $\mathrm{ArC} \underline{\mathrm{H}}), 6.53$ (1H, ddd, $J=7.5,7.5$ and 1.5, $\mathrm{ArC} \underline{\mathrm{H}}), 7.09-7.32(11 \mathrm{H}, \mathrm{m}, \mathrm{ArC} \underline{\mathrm{H}}), 7.41$ $(1 \mathrm{H}, \mathrm{dd}, J=8.0$ and $1.5, \operatorname{ArC} \underline{\mathrm{H}})$, only one signal attributable to $\mathrm{N} \underline{\mathrm{H}}$ was observed; $\delta_{\mathrm{C}}(100$ MHz, $\left.\mathrm{CDCl}_{3}\right) 39.3$ (C1- $\left.\underline{\mathrm{CH}}_{2} \mathrm{Ph}\right), 45.6$ (ㄷ-2), 50.8 ( $\left.\mathrm{NCH}_{2} \mathrm{Ph}\right), 57.0$ (ㄷ-1), 110.0 (ArC), 111.4, 117.4, 126.5, 127.0, 128.1, 128.4 (2 signals), 128.6, 129.2 and $132.3(\operatorname{Ar} \underline{\mathrm{C}} \mathrm{H} \times 14), 138.4$, 140.1 and $145.3(\mathrm{ArC} \times 3)$; HRMS: $\left(\mathrm{ESI}^{+}\right)$Found: $[\mathrm{M}+\mathrm{H}]^{+} 395.1110, \mathrm{C}_{22} \mathrm{H}_{24} \mathrm{~N}_{2}{ }^{79} \mathrm{Br}$ requires 395.1117.

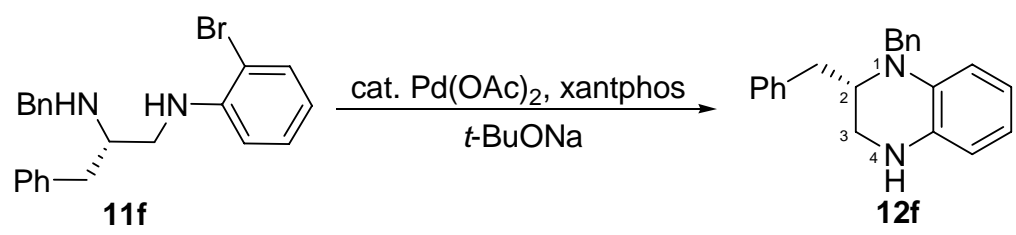

(S)-1,2-Dibenzyl-1,2,3,4-tetrahydroquinoxaline (12f): $\mathrm{Pd}(\mathrm{OAc})_{2}$ (1.5 mg, $\left.7 \mathrm{~mol} \%\right)$, xantphos (3.8 mg, $7 \mathrm{~mol} \%$ ), $t$-BuONa (15 mg, $0.15 \mathrm{mmol}$ ) and amine 11f (38 mg, 0.10 mmol) were dissolved in anhydrous PhMe (1.4 mL), placed in a re-sealable tube and heated at $100{ }^{\circ} \mathrm{C}$ for $72 \mathrm{~h}$. The mixture was cooled to r.t., diluted with EtOAc $(20 \mathrm{~mL})$, washed with water $(10 \mathrm{~mL})$ and then brine $(10 \mathrm{~mL})$, dried $\left(\mathrm{Na}_{2} \mathrm{SO}_{4}\right)$ and concentrated in vacuo. The residue was purified by FCC (EtOAc-hexanes 1:4) to afford benzoxazine $\mathbf{1 2 f}$ (7 mg, $22 \%$ ) as a yellow oil. Continued elution afforded recovered amine $11 \mathrm{f}(10 \mathrm{mg}, 25 \%)$ as a colourless oil.

Data for 12f: $[\alpha]_{\mathrm{D}}^{20}-3.6$ (c = 1.1, $\left.\mathrm{CHCl}_{3}\right) ; v_{\max } / \mathrm{cm}^{-1}$ (film) $2919(\mathrm{~m}), 1601$ (m), 1509 (s), $1452(\mathrm{~m}), 1301(\mathrm{~s}) ; \delta_{\mathrm{H}}\left(400 \mathrm{MHz}, \mathrm{CDCl}_{3}\right) 2.90\left(1 \mathrm{H}, \mathrm{dd}, J=13.0\right.$ and 9.5, C2-C $\left.\underline{H}_{2} \mathrm{Ph}\right), 2.96$ (1H, dd, $J=13.0$ and 6.5, C2- $\left.\underline{\mathrm{H}}_{2} \mathrm{Ph}\right), 3.11$ (1H, dd, $J=11.0$ and 2.0, C3- $\underline{\mathrm{H}}$ ), 3.29 (1H, dd, $J$ $=11.0$ and 3.0, C3- $\underline{\mathrm{H}}), 3.50-3.56(1 \mathrm{H}, \mathrm{m}, \mathrm{C} 2-\underline{\mathrm{H}}), 3.74(1 \mathrm{H}, \mathrm{br} \mathrm{s}, \underline{\mathrm{H}}), 4.33(1 \mathrm{H}, \mathrm{d}, J=16.5$, $\left.\mathrm{NC}_{2} \mathrm{Ph}\right), 4.53\left(1 \mathrm{H}, \mathrm{d}, J=16.5, \mathrm{NC}_{2} \mathrm{Ph}\right), 6.47-6.51(1 \mathrm{H}, \mathrm{m}, \mathrm{ArC} \underline{\mathrm{H}}), 6.57-6.65(3 \mathrm{H}, \mathrm{m}$, $\operatorname{ArC} \underline{\mathrm{H}})$, 7.12-7.33 (10H, m, $\mathrm{ArC} \underline{\mathrm{H}}) ; \delta_{\mathrm{C}}\left(100 \mathrm{MHz}, \mathrm{CDCl}_{3}\right) 37.2$ (C2- $\left.\underline{\mathrm{CH}}_{2} \mathrm{Ph}\right), 41.7$ (ㄷ-3), 54.2 $\left(\mathrm{NCH}_{2} \mathrm{Ph}\right), 58.6$ (ㄷ-2), 112.4, 113.8, 117.4, 119.1, 126.1, 127.0, 128.4, 128.6 and 129.5 $(\mathrm{Ar} \underline{\mathrm{CH}} \times 14), 133.6,133.9,138.8$ and $139.3(\mathrm{ArC} \times 4) ; \mathrm{m} / \mathrm{z}\left(\mathrm{CI}^{+}\right) 315\left([\mathrm{M}+\mathrm{H}]^{+}, 100 \%\right)$; HRMS: $\left(\mathrm{CI}^{+}\right)$Found: $[\mathrm{M}+\mathrm{H}]^{+} 315.1860, \mathrm{C}_{22} \mathrm{H}_{23} \mathrm{~N}_{2}$ requires 315.1861 . 


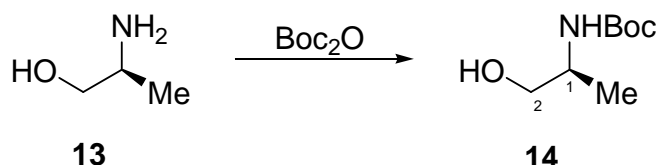

((S)-2-Hydroxy-1-methylethyl)carbamic acid tert-butyl ester (14): To an ice-cooled $\left(0{ }^{\circ} \mathrm{C}\right)$ solution of L-(+)-alaninol 13 (1.50 g, $20.0 \mathrm{mmol})$ and $\mathrm{Et}_{3} \mathrm{~N}(2.78 \mathrm{~mL}, 20.0 \mathrm{mmol})$ in THF (60 $\mathrm{mL}$ ) was added $\mathrm{Boc}_{2} \mathrm{O}$ (4.36 g, $\left.20.0 \mathrm{mmol}\right)$. The mixture was warmed to r.t., stirred for $1.5 \mathrm{~h}$ and then concentrated in vacuo. The residue was dissolved in EtOAc $(50 \mathrm{~mL})$, washed with water $(2 \times 50 \mathrm{~mL})$, brine $(50 \mathrm{~mL})$, dried $\left(\mathrm{Na}_{2} \mathrm{SO}_{4}\right)$ and concentrated in vacuo to afford $N$-Boc alaninol 14 (3.46 g, $99 \%$ ) as a colourless, crystalline solid; m.p. 57-58 ${ }^{\circ} \mathrm{C}$ (EtOAc-hexanes) [Lit., ${ }^{3} 57-58{ }^{\circ} \mathrm{C}$ (EtOAc-petrol)]; $[\alpha]_{\mathrm{D}}{ }^{20}-4.2$ (c $\left.=1.8, \mathrm{CHCl}_{3}\right)\left[\right.$ Lit., $^{3}[\alpha]_{\mathrm{D}}{ }^{24}-6.9$ (c $=0.3$, $\left.\left.\mathrm{CHCl}_{3}\right)\right] ; \delta_{\mathrm{H}}\left(400 \mathrm{MHz}, \mathrm{CDCl}_{3}\right) 1.15\left(3 \mathrm{H}, \mathrm{d}, J=6.5, \mathrm{C} 1-\mathrm{CH}_{3}\right), 1.45\left(9 \mathrm{H}, \mathrm{s}, \mathrm{NCO}_{2} \mathrm{C}\left(\mathrm{CH}_{3}\right)_{3}\right)$, $2.66(1 \mathrm{H}, \mathrm{br} \mathrm{s}, \mathrm{O} \underline{\mathrm{H}}), 3.46-3.55(1 \mathrm{H}, \mathrm{m}, \mathrm{C} 2-\underline{\mathrm{H}}), 3.61-3.68(1 \mathrm{H}, \mathrm{m}, \mathrm{C} 2-\underline{\mathrm{H}}), 3.71-3.84(1 \mathrm{H}, \mathrm{m}$, $\mathrm{C} 1-\underline{\mathrm{H}}), 4.67(1 \mathrm{H}, \mathrm{br} \mathrm{s}, \mathrm{N} \underline{\mathrm{H}})$. The spectroscopic properties of this compound were consistent with the data available in the literature. ${ }^{3}$

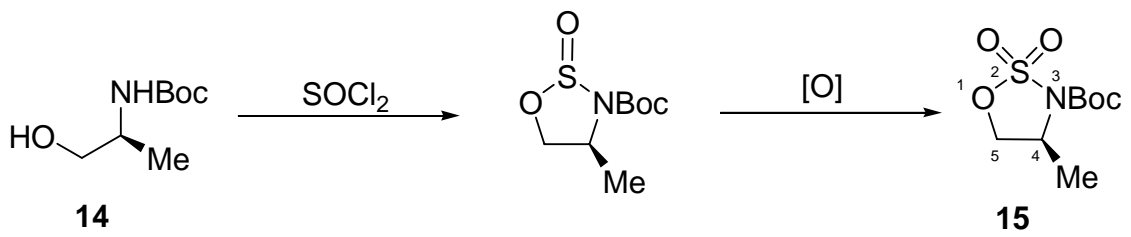

(S)-4-Methyl-2,2-dioxo-2 $\lambda^{6}$-[1,2,3]oxathiazolidine-3-carboxylic acid tert-butyl ester (15): To a cooled $\left(-50{ }^{\circ} \mathrm{C}\right)$ solution of $\mathrm{SOCl}_{2}(468 \mu \mathrm{L}, 6.41 \mathrm{mmol})$, imidazole (1.53 g, $\left.22.5 \mathrm{mmol}\right)$ and $\mathrm{Et}_{3} \mathrm{~N}(1.71 \mathrm{~mL}, 12.3 \mathrm{mmol})$ in anhydrous $\mathrm{CH}_{2} \mathrm{Cl}_{2}(57 \mathrm{~mL})$ was added dropwise, via syringe pump, a solution of $N$-Boc alaninol 14 (1.00 g, $5.71 \mathrm{mmol})$ in anhydrous $\mathrm{CH}_{2} \mathrm{Cl}_{2}(7$ $\mathrm{mL}$ ) over $0.5 \mathrm{~h}$. The mixture was then warmed to $0{ }^{\circ} \mathrm{C}$ and stirred for $21 \mathrm{~h}$ prior to the addition of water $(50 \mathrm{~mL})$. The organic portion was isolated, washed with brine $(50 \mathrm{~mL})$, dried $\left(\mathrm{Na}_{2} \mathrm{SO}_{4}\right)$ and concentrated in vacuo to afford intermediate cyclic sulfamidite (1.27 g, $100 \%)$ as a colourless oil. This material was used immediately in the next stage without further purification. To an ice-cooled $\left(0{ }^{\circ} \mathrm{C}\right)$ solution of intermediate cyclic sulfamidite $(1.23$ g, $5.58 \mathrm{mmol}$ ) in MeCN (45 mL) was added sequentially $\mathrm{NaIO}_{4}(1.31 \mathrm{~g}, 6.14 \mathrm{mmol}), \mathrm{RuCl}_{3}$ (1.1 mg, $0.1 \mathrm{~mol} \%$ ) and then water $(35 \mathrm{~mL})$. The mixture was stirred at $0{ }^{\circ} \mathrm{C}$ for $2.25 \mathrm{~h}$ and then diluted with water $(80 \mathrm{~mL})$ and extracted with $\mathrm{Et}_{2} \mathrm{O}(2 \times 150 \mathrm{~mL})$. The organic extracts were combined, washed with water $(150 \mathrm{~mL})$ and then brine $(150 \mathrm{~mL})$, dried $\left(\mathrm{Na}_{2} \mathrm{SO}_{4}\right)$ and concentrated in vacuo. The residue was filtered through a pad of $\mathrm{SiO}_{2}\left(60\right.$, eluting with $\mathrm{Et}_{2} \mathrm{O}$ ) to afford cyclic sulfamidate 15 (1.19 g, 90 \%, 4:1 rotamer ratio) as a colourless, crystalline solid; m.p. 118-120 ${ }^{\circ} \mathrm{C}$ (EtOAc-hexanes) [Lit., ${ }^{4}$ 120-121 ${ }^{\circ} \mathrm{C}$ (no recrystallisation solvent quoted)]; $[\alpha]_{\mathrm{D}}^{20}+11.1(\mathrm{c}=1.0, \mathrm{MeOH})\left[\right.$ Lit., $\left.{ }^{4}[\alpha]_{\mathrm{D}}{ }^{24}+9.45(\mathrm{c}=1.06, \mathrm{MeOH})\right] ; \delta_{\mathrm{H}}(400 \mathrm{MHz}$, 
$\mathrm{CDCl}_{3}$ ) (data for major rotamer only) $1.50\left(3 \mathrm{H}, \mathrm{d}, J=6.5, \mathrm{C} 4-\mathrm{CH}_{3}\right), 1.53(9 \mathrm{H}, \mathrm{s}$, $\left.\mathrm{NCO}_{2} \mathrm{C}\left(\underline{\mathrm{C}}_{3}\right)_{3}\right), 4.00-4.10$ (1H, m, C4- $\left.\underline{\mathrm{H}}\right), 4.68(1 \mathrm{H}, \mathrm{dd}, J=9.5$ and 9.0, C3- $\underline{\mathrm{H}}), 4.79(1 \mathrm{H}, \mathrm{dd}$, $J=9.0$ and 7.0, C3- $\underline{\mathrm{H}})$. The spectroscopic properties of this compound were consistent with the data available in the literature. ${ }^{4}$

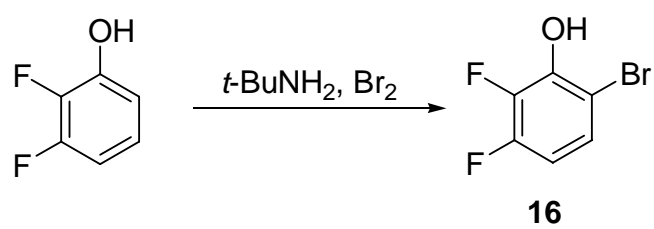

6-Bromo-2,3-difluorophenol (16): To a cooled $\left(-30{ }^{\circ} \mathrm{C}\right)$ solution of $t-\mathrm{BuNH}_{2}(1.62 \mathrm{~mL}, 15.3$ mmol) in anhydrous PhMe (19 mL) was added $\mathrm{Br}_{2}(395 \mu \mathrm{L}, 7.69 \mathrm{mmol})$ dropwise over 3 minutes to form a yellow solution. The mixture was cooled to $-78{ }^{\circ} \mathrm{C}$ and, after a further 10 minutes, a solution of 2,3-difluorophenol (1.00 g, $7.69 \mathrm{mmol})$ in anhydrous $\mathrm{CH}_{2} \mathrm{Cl}_{2}(2 \mathrm{~mL})$ was added dropwise over 5 minutes. The mixture was allowed to warm slowly to r.t. over $4 \mathrm{~h}$ and was then stirred at this temperature for a further $1.5 \mathrm{~h}$. The mixture was diluted with EtOAc $(20 \mathrm{~mL})$, washed with aq. $1 \mathrm{M} \mathrm{HCl}(2 \times 20 \mathrm{~mL})$, dried $\left(\mathrm{Na}_{2} \mathrm{SO}_{4}\right)$ and concentrated in vacuo to afford a brown oil. This residue was purified by FCC (hexanes-EtOAc 5:1) to afford phenol 16 (1.46 g, $91 \%$ ) as a yellow oil; $v_{\max } / \mathrm{cm}^{-1}$ (film) 3502 (br m), 1707 (m), 1620 (m), 1479 (s), 1463 (s), 1319 (m), 1289 (s), 1234 (s), 1181 (s), 1108 (m), 1025 (s); $\delta_{\text {H }}(400$ MHz, $\left.\mathrm{CDCl}_{3}\right) 5.80$ (1H, br s, $\left.\mathrm{O} \underline{\mathrm{H}}\right), 6.72(1 \mathrm{H}, \mathrm{ddd}, J=9.5,9.0$ and 7.5, $\mathrm{ArC} \underline{\mathrm{H}}), 7.23$ (1H, ddd, $J=$ 9.0, 5.0 and 2.5, $\mathrm{ArC} \underline{\mathrm{H}}$ ); $\delta_{\mathrm{C}}\left(100 \mathrm{MHz}, \mathrm{CDCl}_{3}\right) 105.3$ (d, $J=3.0, \mathrm{ArC}$ ), 109.3 (d, $J=19.0$,

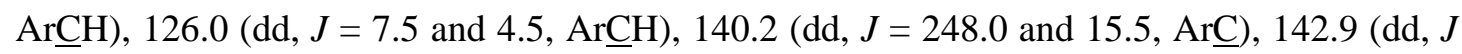
= 11.5 and 3.5, $\operatorname{Ar} \underline{C}), 150.6$ (dd, $J=252.0$ and 12.5, $\operatorname{Ar} \underline{C}) ; m / z\left(\mathrm{CI}^{+}\right) 209$ and $211\left([\mathrm{M}+\mathrm{H}]^{+}\right.$, 100 and 95 \%); HRMS: $\left(\mathrm{CI}^{+}\right)$Found: $[\mathrm{M}+\mathrm{H}]^{+}$208.9409, $\mathrm{C}_{6} \mathrm{H}_{4} \mathrm{OF}_{2}{ }^{79} \mathrm{Br}$ requires 208.9414.

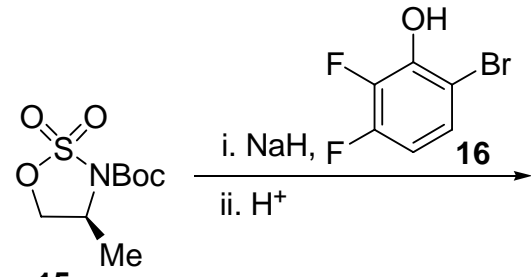

15

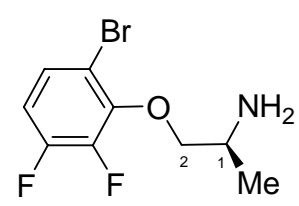

18

(S)-2-(6-Bromo-2,3-difluorophenoxy)-1-methylethylamine (18): To a solution of phenol 16 (211 mg, $1.01 \mathrm{mmol})$ in anhydrous DMF $(9.7 \mathrm{~mL}$ ) was added $\mathrm{NaH}$ (60\% dispersion in mineral oil, $40 \mathrm{mg}, 1.01 \mathrm{mmol}$ ) and the resulting mixture was stirred at r.t. for 5 minutes. Cyclic sulfamidate 15 (200 mg, $0.84 \mathrm{mmol}$ ) was added and the mixture was stirred at r.t. for $15 \mathrm{~h}$ prior to concentration in vacuo. The residue was suspended in $p$-dioxane $(5.5 \mathrm{~mL})$, water $(100 \mu \mathrm{L})$ and c. $\mathrm{H}_{2} \mathrm{SO}_{4}(100 \mu \mathrm{L})$ were added and the mixture was stirred at r.t. for $0.5 \mathrm{~h}$. 
Further c. $\mathrm{H}_{2} \mathrm{SO}_{4}(0.55 \mathrm{~mL})$ was added and the mixture was stirred at r.t. for 0.5 h. The mixture was neutralised with saturated aq. $\mathrm{NaHCO}_{3}$ and extracted with $\mathrm{CH}_{2} \mathrm{Cl}_{2}(3 \times 20 \mathrm{~mL})$. The combined organic extracts were concentrated in vacuo to afford amine 18 (221 mg, 99 $\%)$ as a colourless oil. This material was suitable for subsequent applications without any further purification but, for analysis, a small portion was isolated by FCC (hexanes-EtOAc 1:0 - 5:1) as a colourless oil; $[\alpha]_{\mathrm{D}}^{20}+24.6\left(\mathrm{c}=1.3, \mathrm{CHCl}_{3}\right) ; v_{\max } / \mathrm{cm}^{-1}($ film) $2928(\mathrm{~m}), 1486$ (s), 1452 (s), 1294 (m), 1206 (m), 1048 (s); $\delta_{\mathrm{H}}\left(400 \mathrm{MHz}, \mathrm{CDCl}_{3}\right) 1.09$ (3H, d, $J=6.5, \mathrm{C} 1-$ $\left.\mathrm{C}_{3}\right), 1.58$ (2H, br s, $\underline{\mathrm{H}}_{2}$ ), 3.30 (1H, dqd, $J=8.0,6.5$ and 3.5, C1- $\left.\underline{\mathrm{H}}\right), 3.76$ (1H, ddd, $J=9.0$, 8.0 and 1.0, C2- $\underline{\mathrm{H}}$ ), 4.05 (1H, ddd, $J=9.0,3.5$ and 1.5, C2- $\underline{\mathrm{H}}), 6.75$ (1H, ddd, $J=9.5,9.0$ and 7.5, $\mathrm{ArC} \underline{\mathrm{H}}), 7.18(1 \mathrm{H}, \mathrm{ddd}, J=9.0,5.5$ and 2.5, $\mathrm{ArC} \underline{\mathrm{H}})$; $\delta_{\mathrm{C}}\left(100 \mathrm{MHz}, \mathrm{CDCl}_{3}\right) 19.3$ (C1-

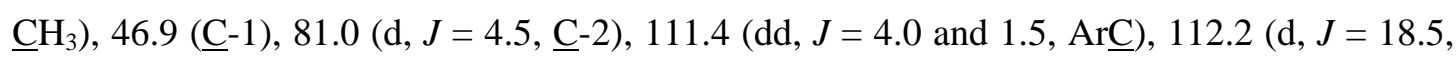

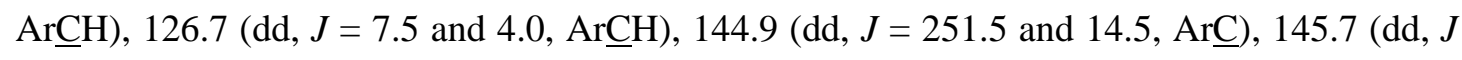
$=10.0$ and 2.5, ArC $), 150.8$ (dd, $J=250.0$ and 11.5, $\operatorname{Ar} \underline{C}) ; \mathrm{m} / \mathrm{z}\left(\mathrm{CI}^{+}\right) 266$ and $268\left([\mathrm{M}+\mathrm{H}]^{+}\right.$, 100 and 90 \%); HRMS: $\left(\mathrm{CI}^{+}\right)$Found: $[\mathrm{M}+\mathrm{H}]^{+}$265.9984, $\mathrm{C}_{9} \mathrm{H}_{11} \mathrm{NOF}_{2}{ }^{79} \mathrm{Br}$ requires 265.9992.

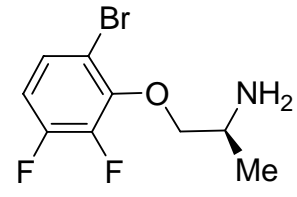

18

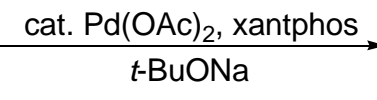
t-BuONa

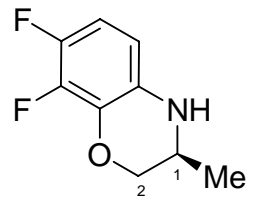

19

(S)-7,8-Difluoro-3-methyl-3,4-dihydro-2H-benzo[1,4]oxazine (19): Amine 18 (60 mg, 0.23 mmol), $\mathrm{Pd}(\mathrm{OAc})_{2}(3.1 \mathrm{mg}, 6 \mathrm{~mol} \%)$, xantphos (7.9 mg, $6 \mathrm{~mol} \%$ ) and $t$-BuONa (28 mg, 0.29 mmol) were dissolved in anhydrous PhMe $(2.5 \mathrm{~mL})$ and placed in a re-sealable tube. The mixture was heated at $100{ }^{\circ} \mathrm{C}$ for $18 \mathrm{~h}$ and then cooled to r.t.. The mixture was then diluted with EtOAc $(20 \mathrm{~mL})$, washed with water $(20 \mathrm{~mL})$ and then brine $(20 \mathrm{~mL})$, dried $\left(\mathrm{Na}_{2} \mathrm{SO}_{4}\right)$ and concentrated in vacuo. The residue was purified by FCC (hexanes-EtOAc 2:1) to afford benzoxazine 19 (36 mg, $84 \%$ ) as a colourless oil; $[\alpha]_{\mathrm{D}}{ }^{20}-9.1\left(\mathrm{c}=1.3, \mathrm{CHCl}_{3}\right)\left[\right.$ Lit., ${ }^{5}[\alpha]_{\mathrm{D}}{ }^{23}-$ 7.8 (c = 0.68, $\left.\mathrm{CHCl}_{3}\right)$ ]; $v_{\max } / \mathrm{cm}^{-1}$ (film) 3387 (br m), 1502 (s), 1316 (m), 1226 (s), 1063 (s), $1045(\mathrm{~m}) ; \delta_{\mathrm{H}}\left(400 \mathrm{MHz}, \mathrm{CDCl}_{3}\right) 1.20$ (3H, d, $\left.J=6.5, \mathrm{C}^{-}-\underline{\mathrm{H}}_{3}\right), 3.51$ (1H, dqd, $J=8.5,6.5$

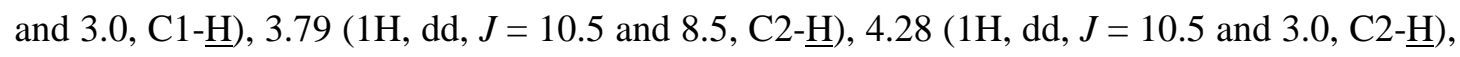
6.26 (1H, ddd, $J=9.0,4.5$ and 2.5, $\operatorname{ArC} \underline{H}), 6.55$ (1H, ddd, $J=10.0,9.0$ and 8.0, $\operatorname{ArC} \underline{H}$ ), a signal attributable to $\mathrm{N} \underline{\mathrm{H}}$ was not observed; $\delta_{\mathrm{C}}\left(100 \mathrm{MHz}, \mathrm{CDCl}_{3}\right) 17.4\left(\mathrm{C} 1-\underline{\mathrm{CH}}_{3}\right), 44.8(\underline{\mathrm{C}}-1)$, 70.9 (ㄷ-2), 107.7 (d, $J=18.5, \operatorname{Ar} \underline{C H}$ ), 108.4 (dd, $J=7.0$ and 3.5, ArㅁH), 130.9 (dd, $J=2.5$

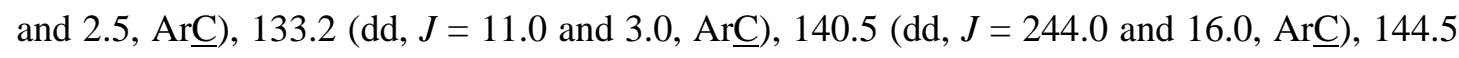
(dd, $J=237.0$ and 11.0, ArC ); $\delta_{\mathrm{F}}\left(283 \mathrm{MHz}, \mathrm{CDCl}_{3}\right.$ ) -160.8 (ddd, $J=21.0,7.5$ and 1.5), 149.9 (ddd, $J=21.0,10.0$ and 5.5); $\mathrm{m} / z\left(\mathrm{CI}^{+}\right) 186\left([\mathrm{M}+\mathrm{H}]^{+}, 100 \%\right)$; HRMS: $\left(\mathrm{CI}^{+}\right)$Found: 
$[\mathrm{M}+\mathrm{H}]^{+}$186.0727, $\mathrm{C}_{9} \mathrm{H}_{10} \mathrm{NOF}_{2}$ requires 186.0730. The spectroscopic properties of this compound were consistent with the data available in the literature. ${ }^{5}$

\section{(C) References relevant to Supporting Information}

1. Blid, J.; Brandt P.; Somfai, P. J. Org. Chem. 2004, 69, 3043-3049.

2. Novak, Z.; Timari G.; Kotschy, A. Tetrahedron 2003, 59, 7509-7513.

3. $\quad$ Sutherland A.; Willis, C. L. J. Org. Chem. 1998, 63, 7764-7769.

4. Posakony, J. J.; Grierson J. R.; Tewson, T. J. J. Org. Chem. 2002, 67, 5164-5169.

5. $\quad$ Atarashi, S.; Yokohama, S.; Yamazaki, K.; Sakano, K.; Imamura M.; Hayakawa, I. Chem. Pharm. Bull. 1987, 35, 1896-1902. 


\section{(D) Copies of ${ }^{1} \mathrm{H}$ and ${ }^{13} \mathrm{C}$ NMR spectra}




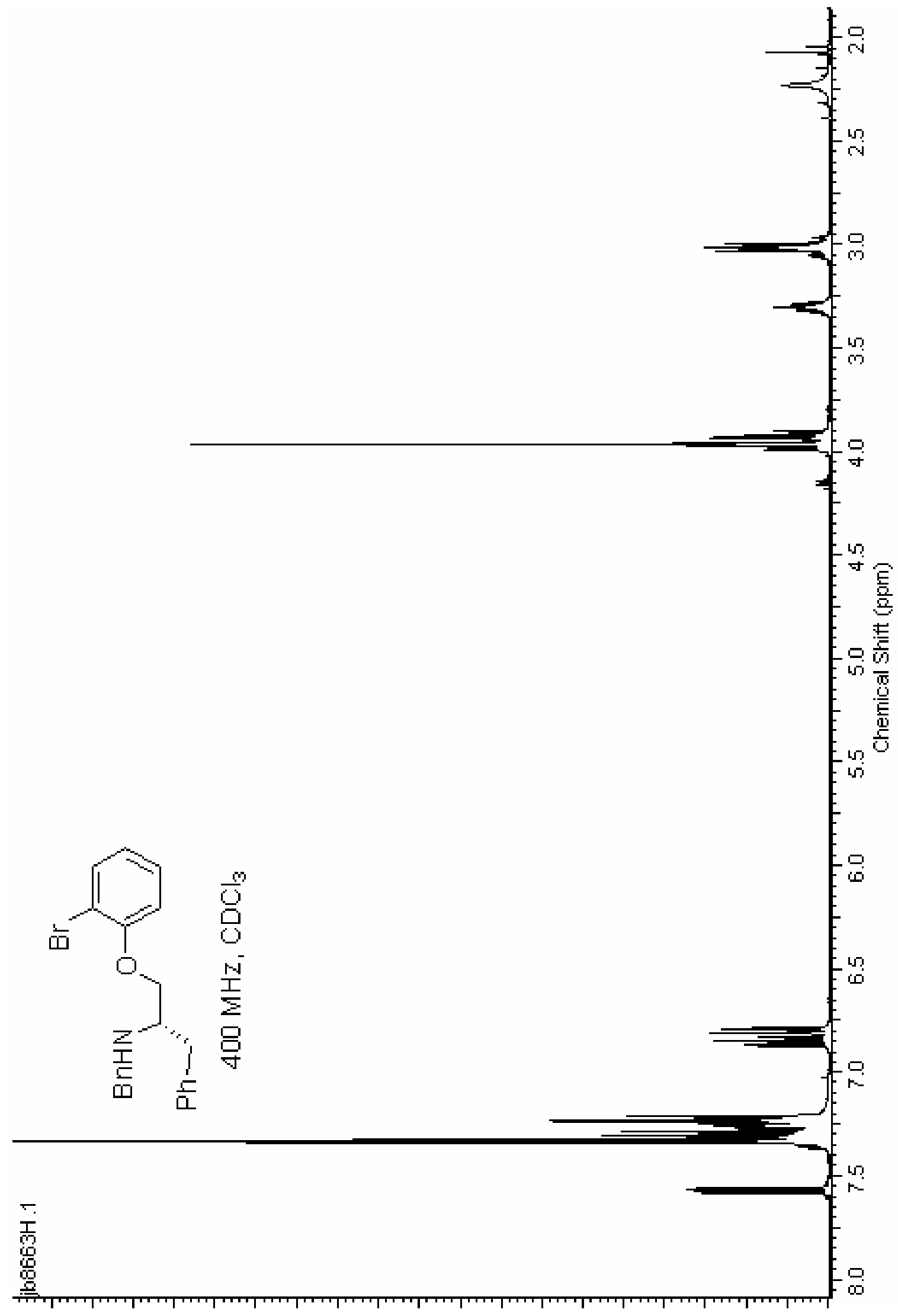




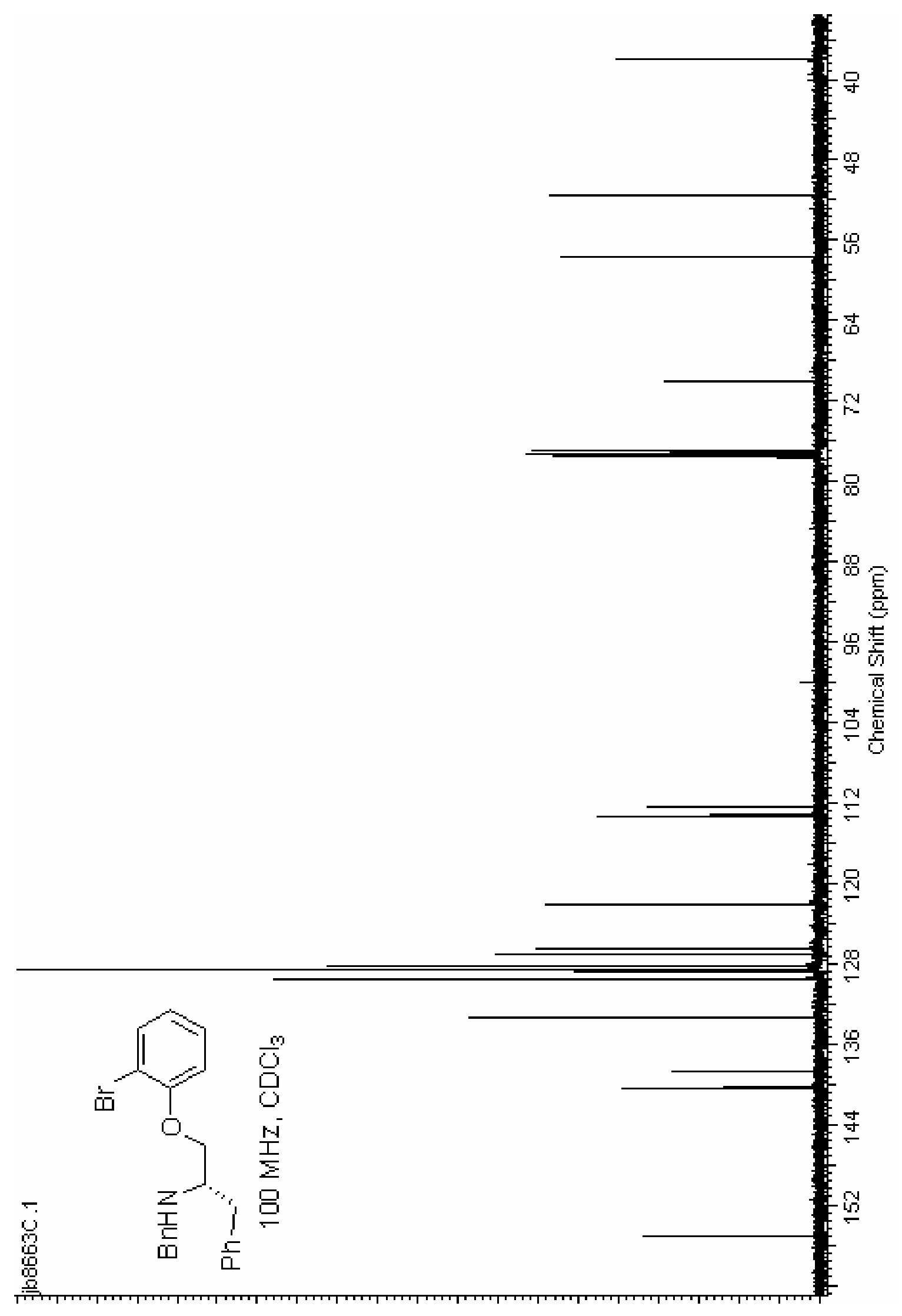




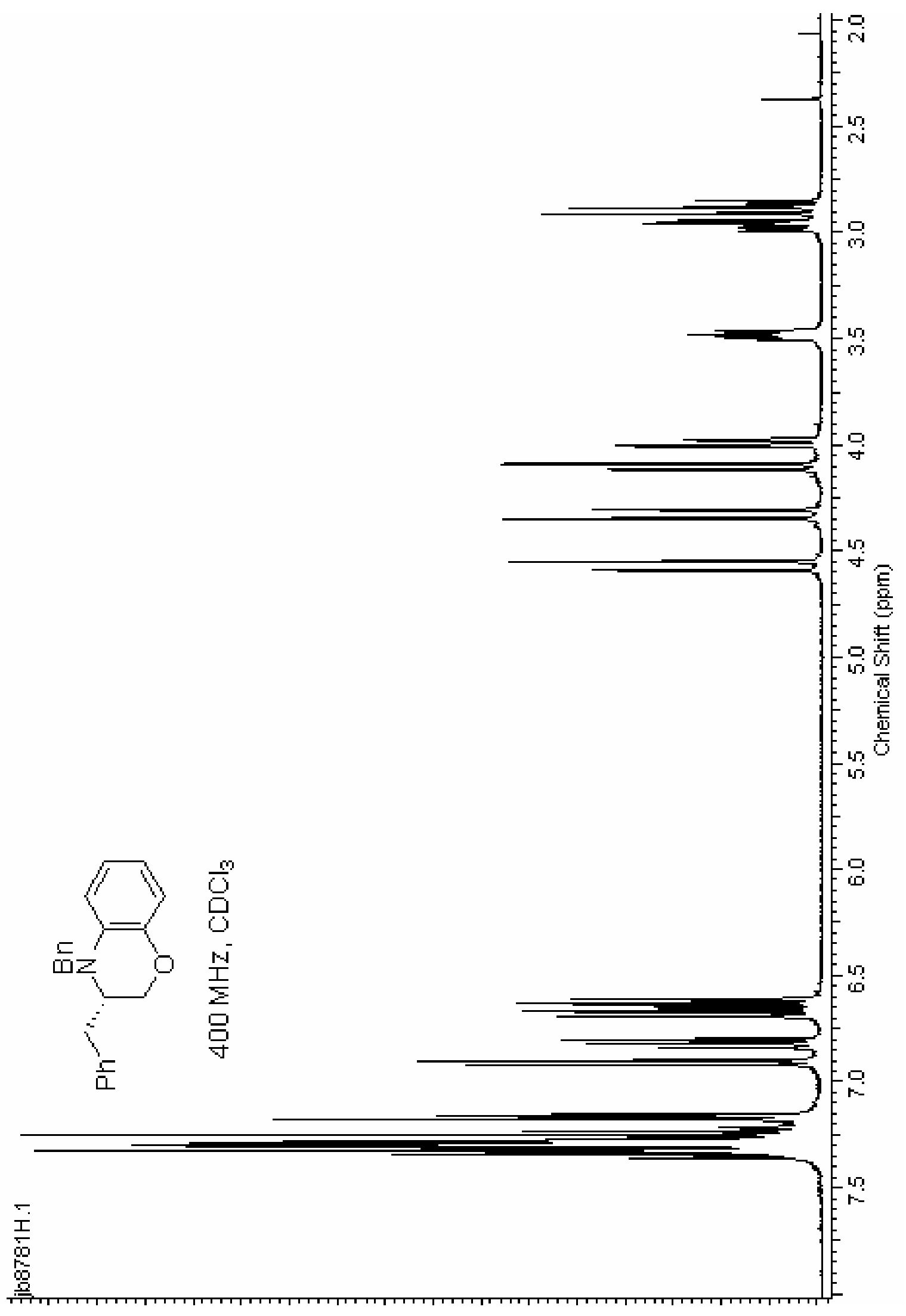




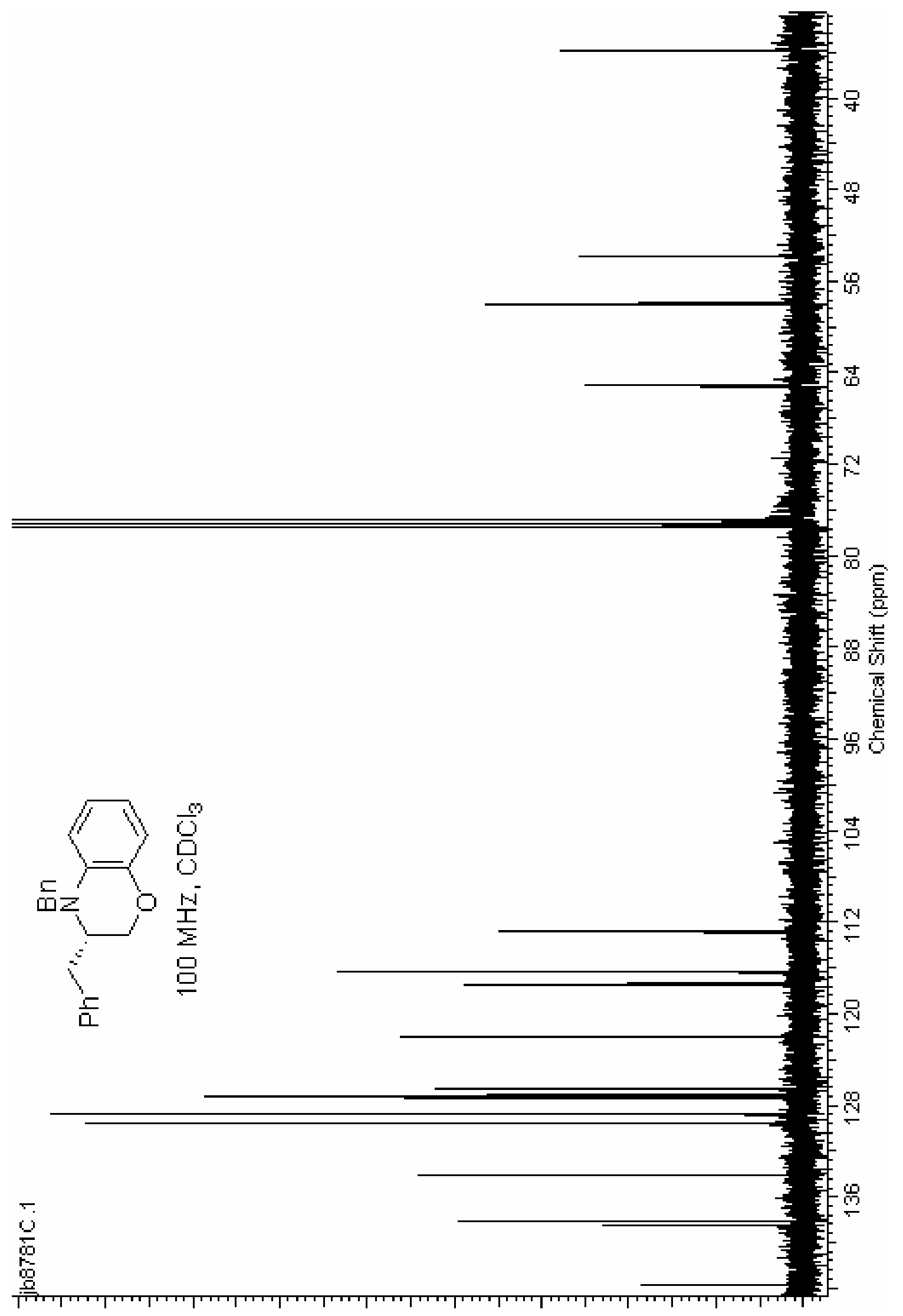




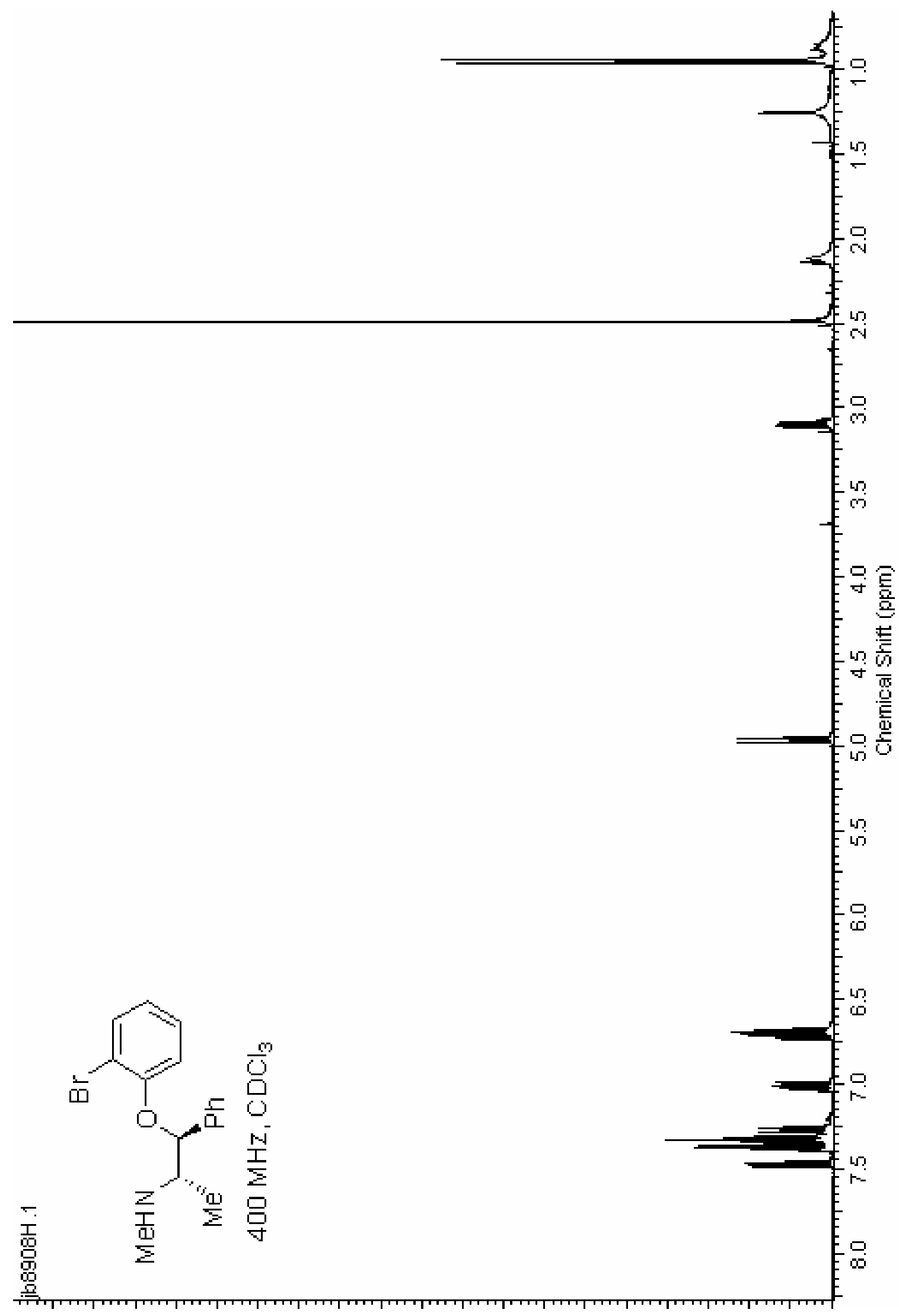




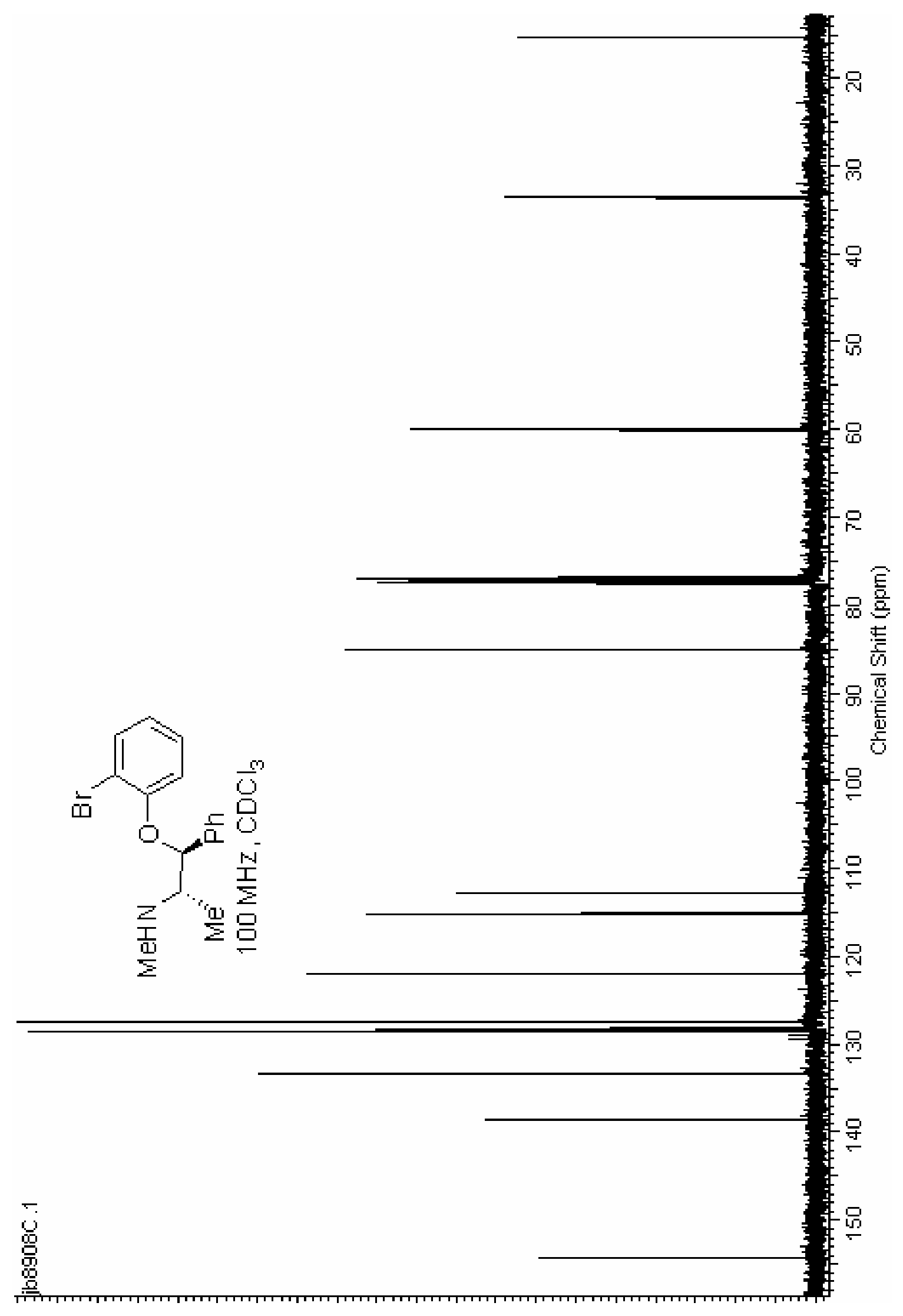




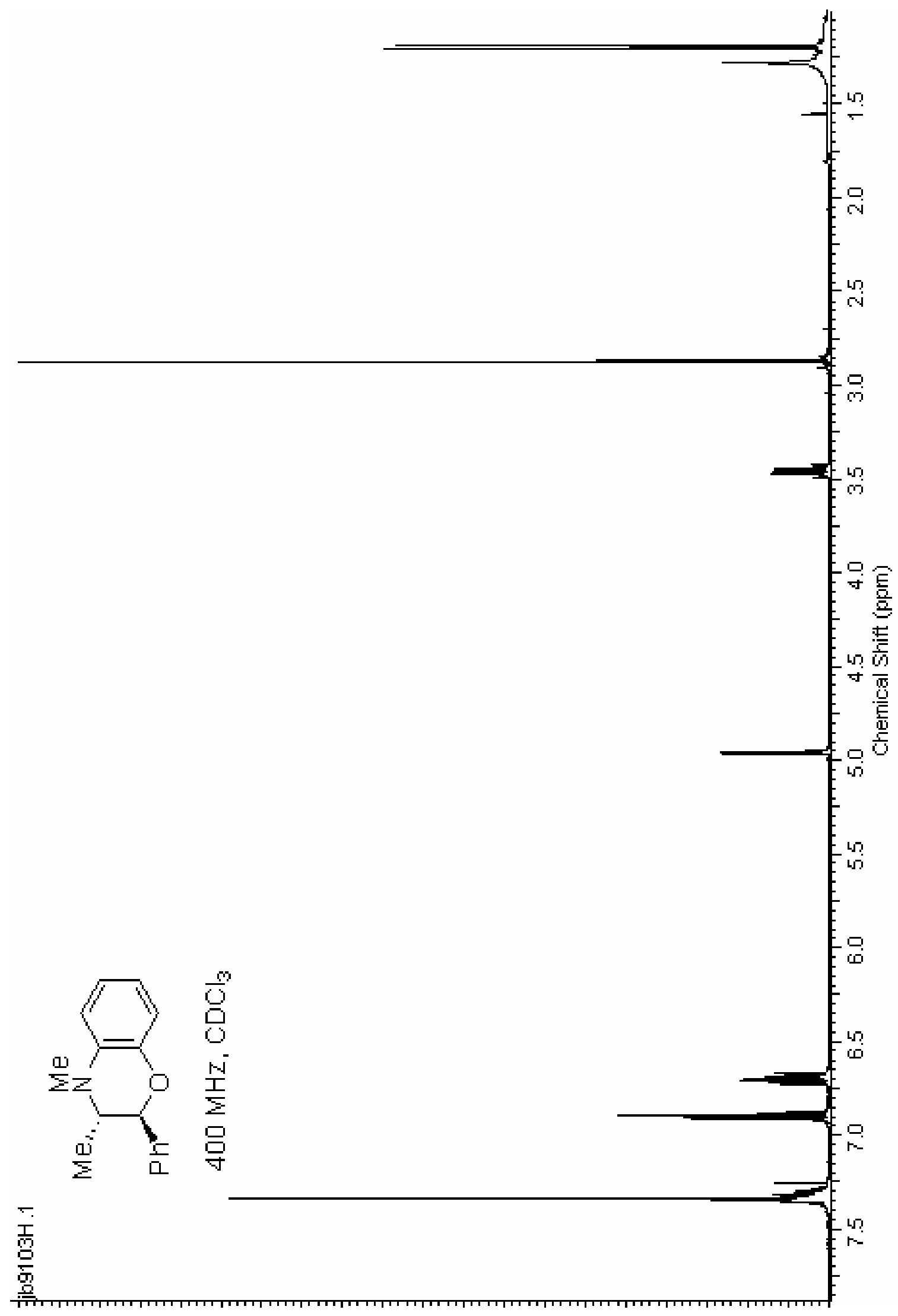




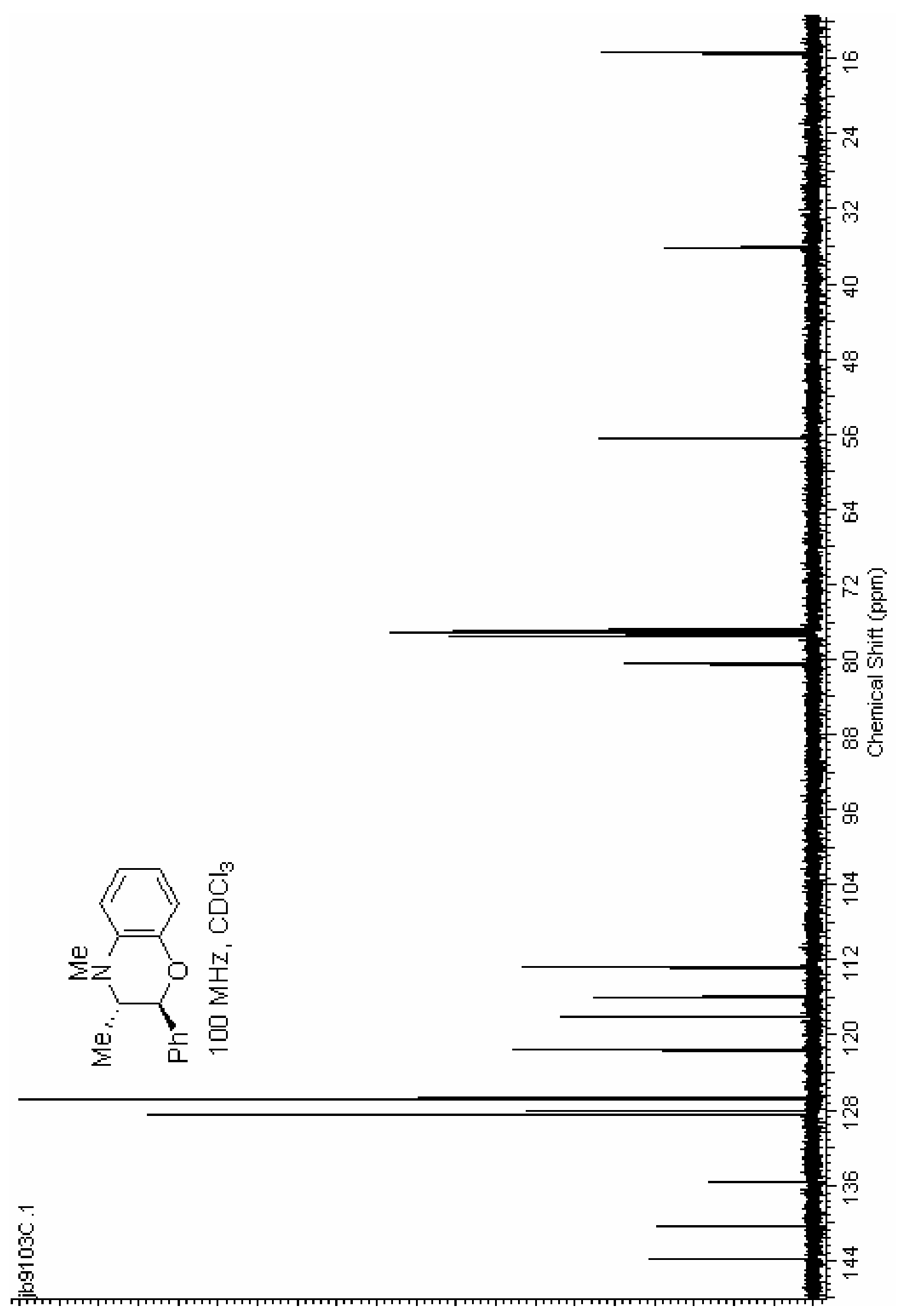




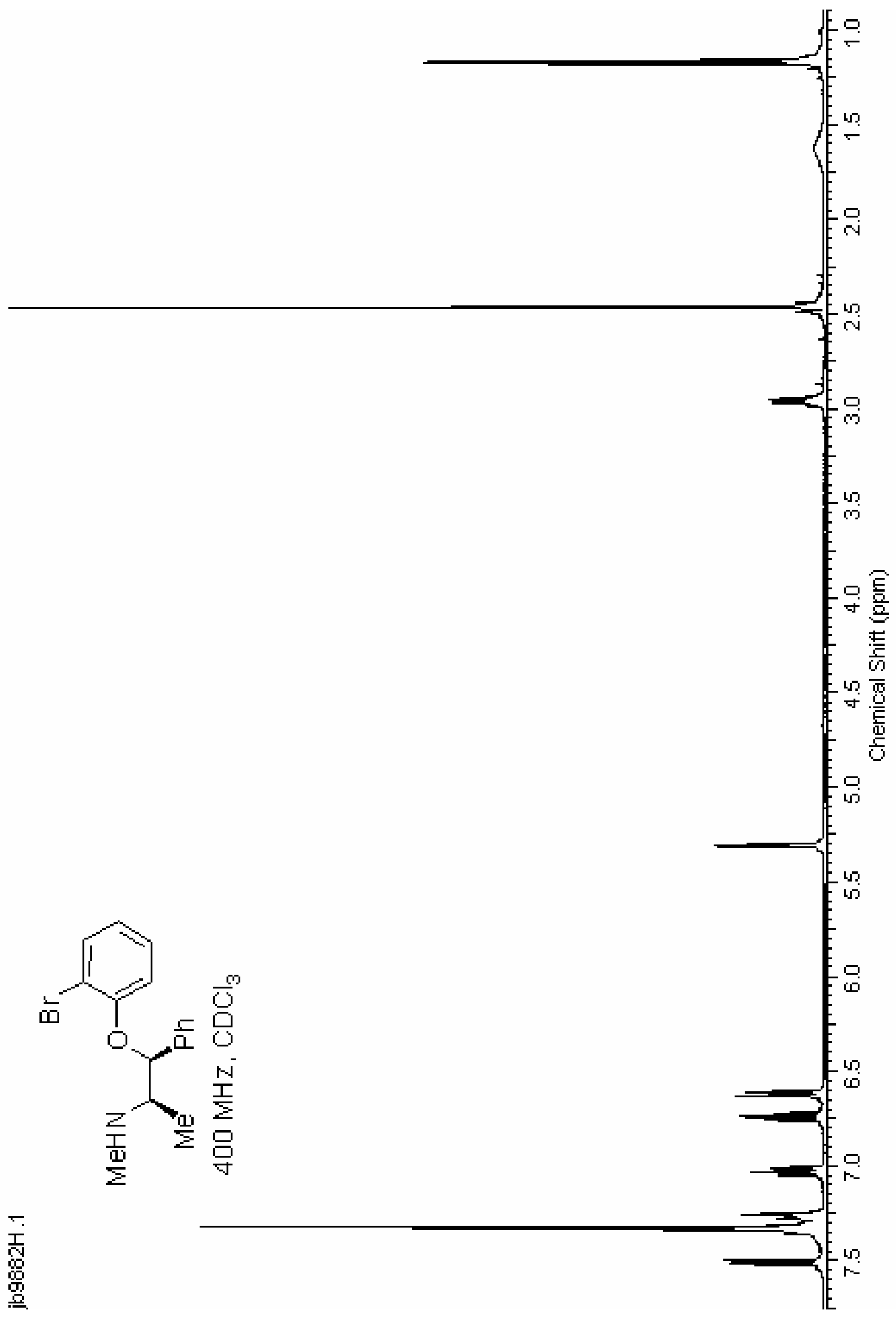




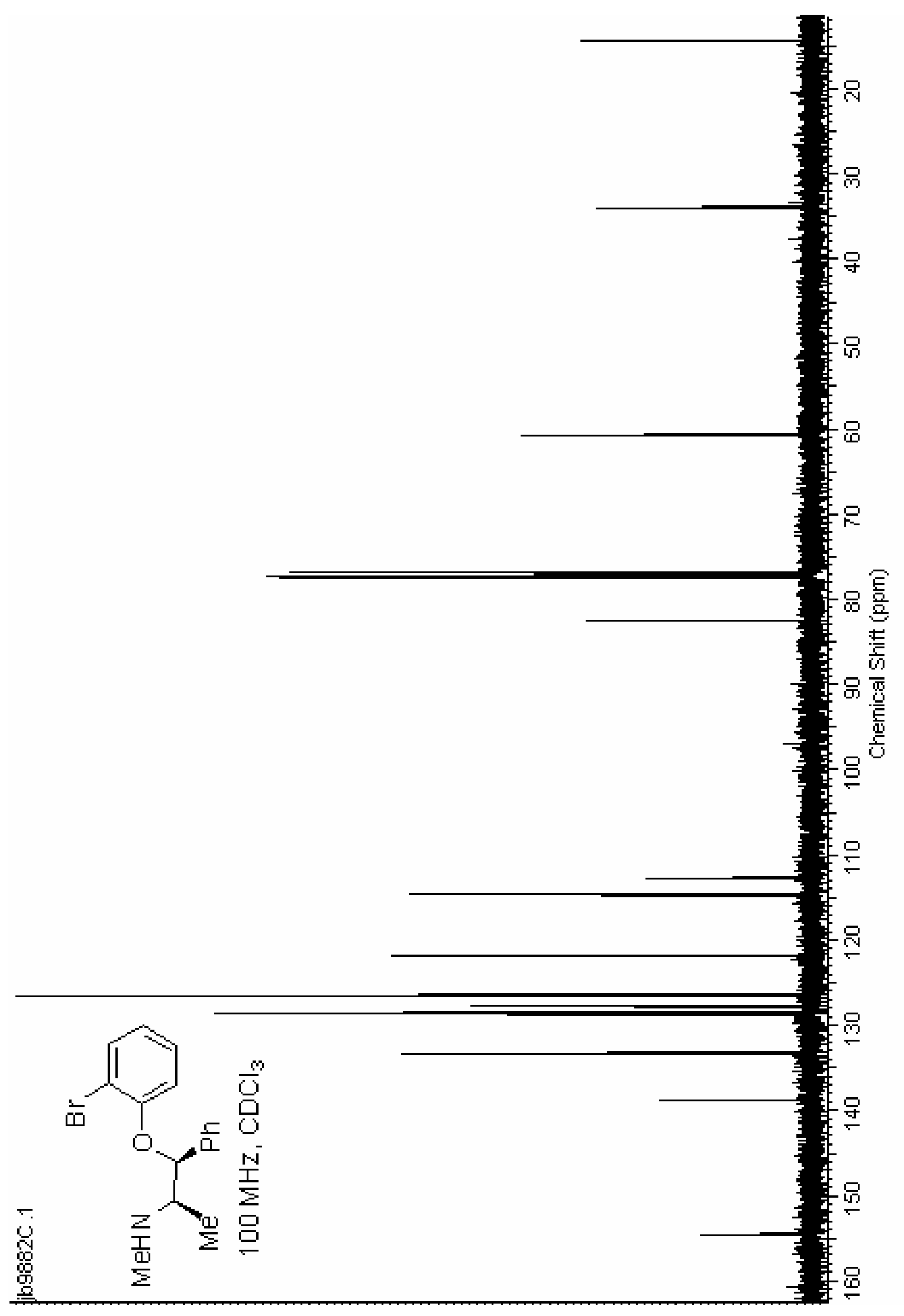




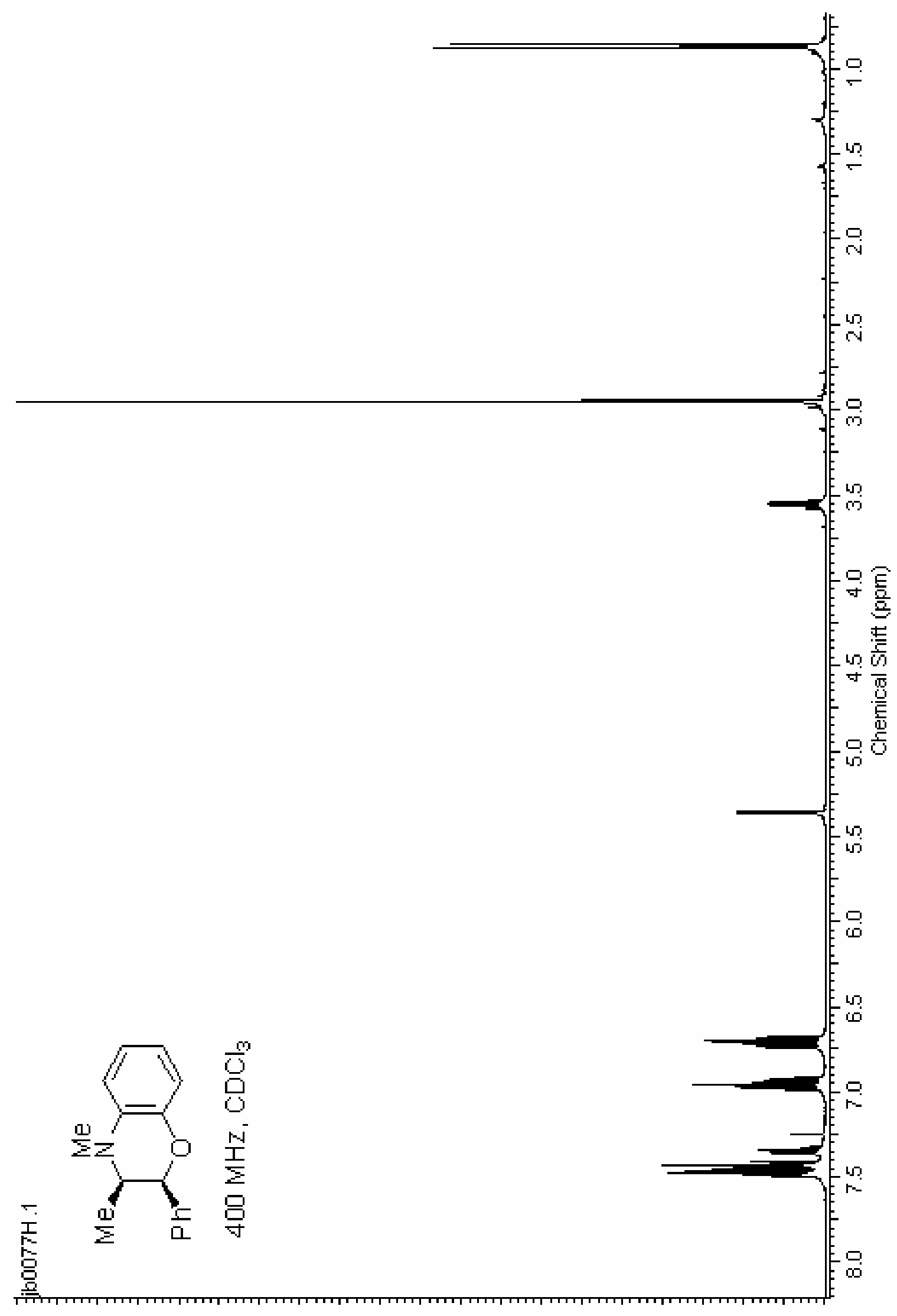




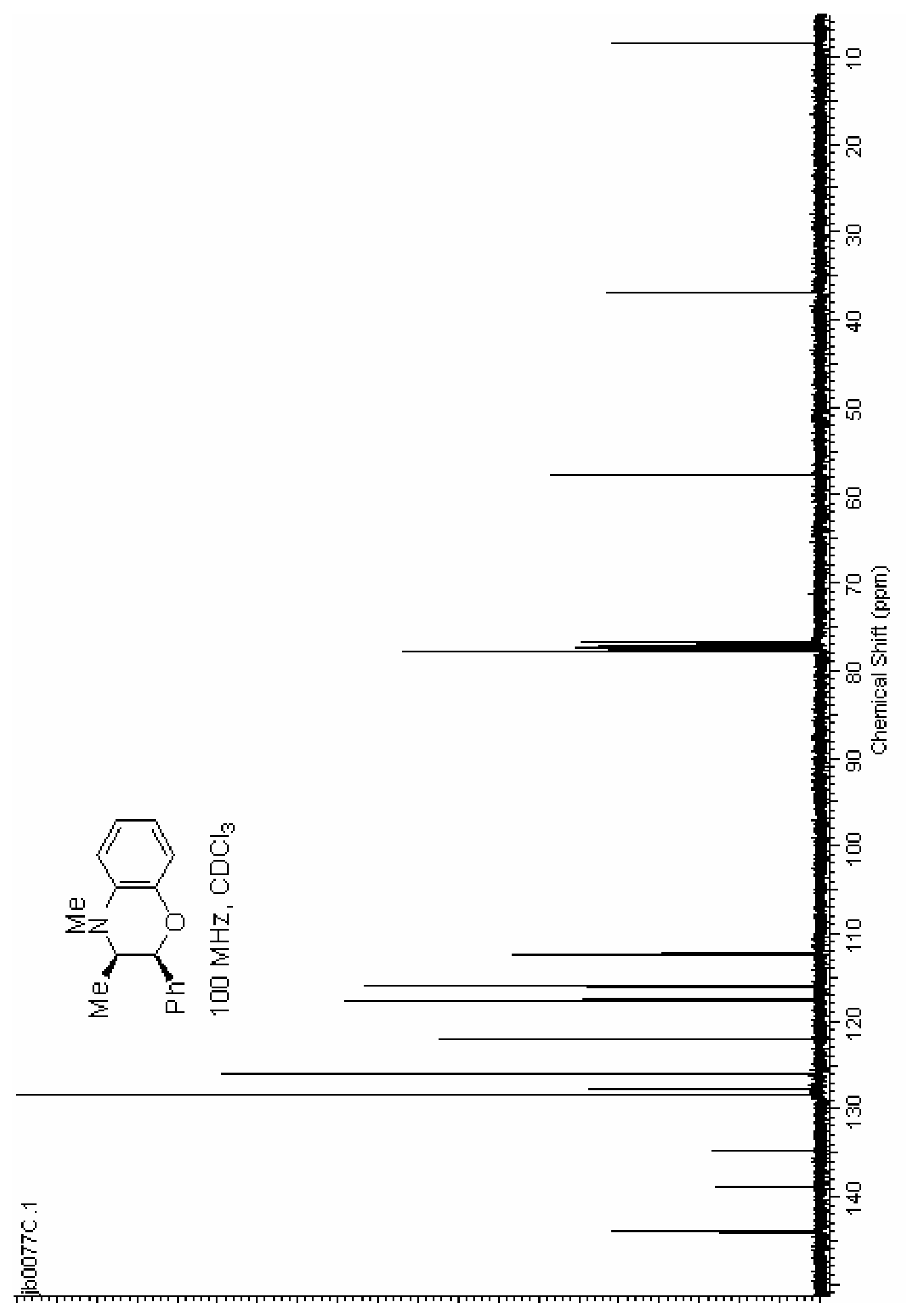




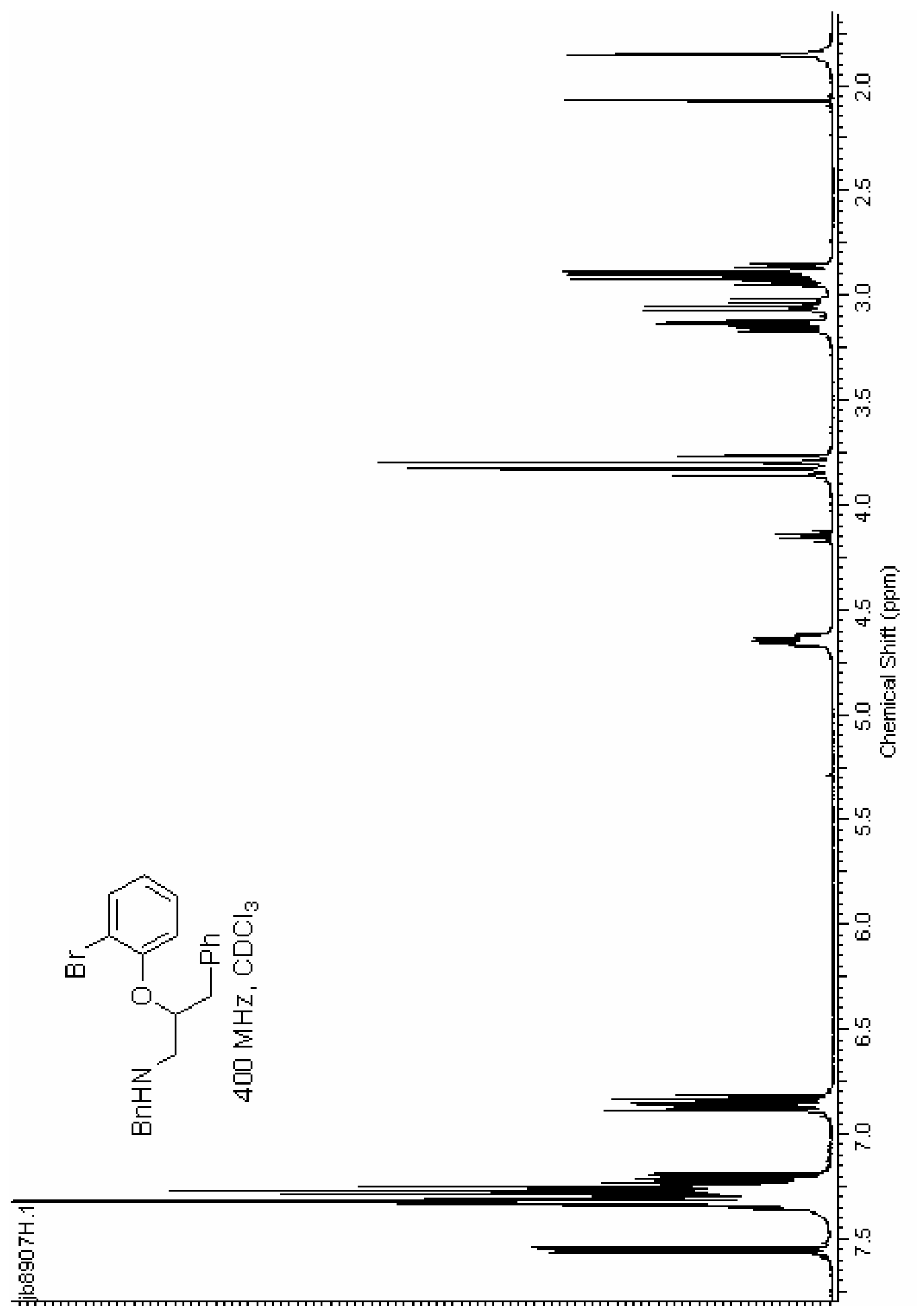




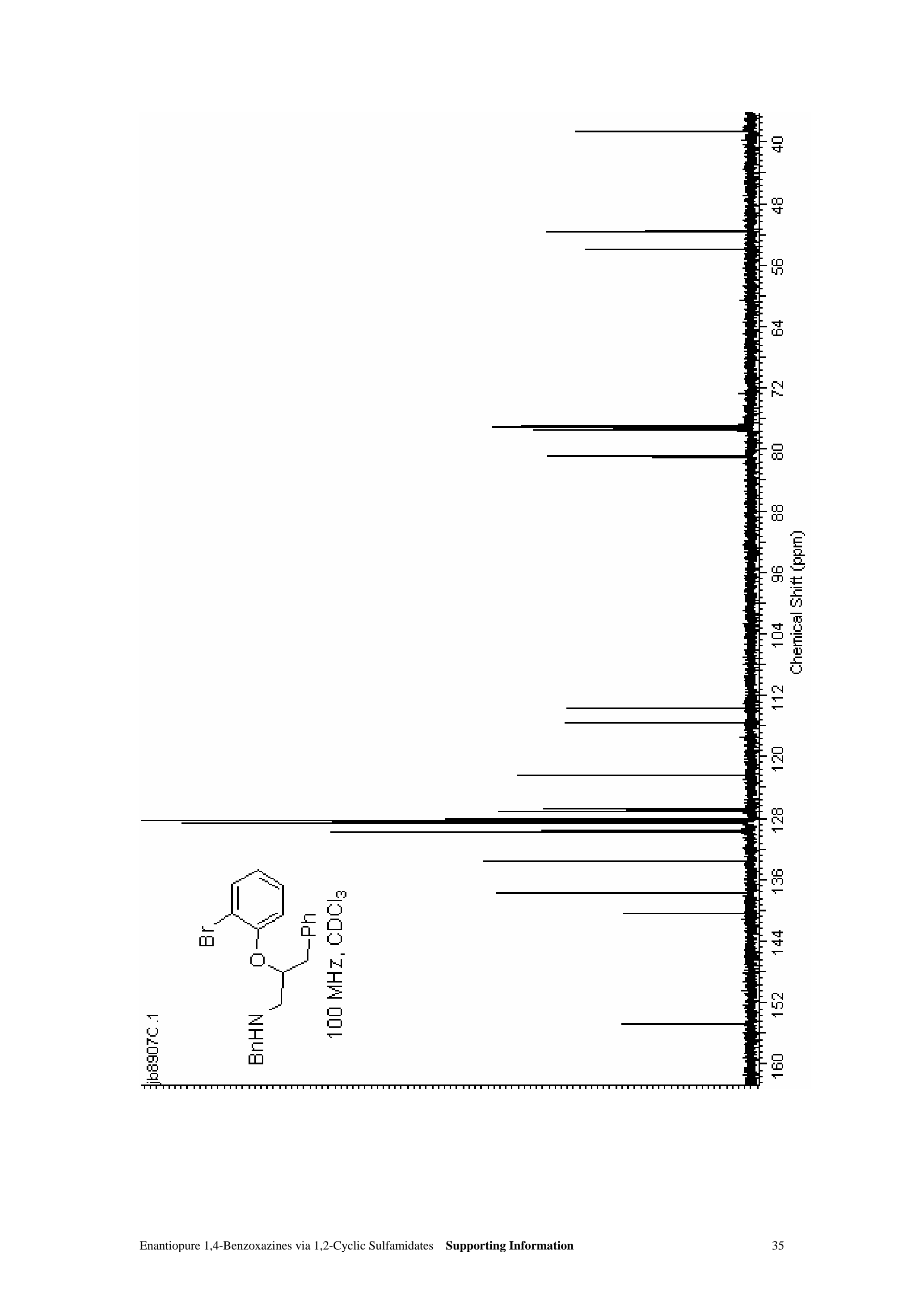




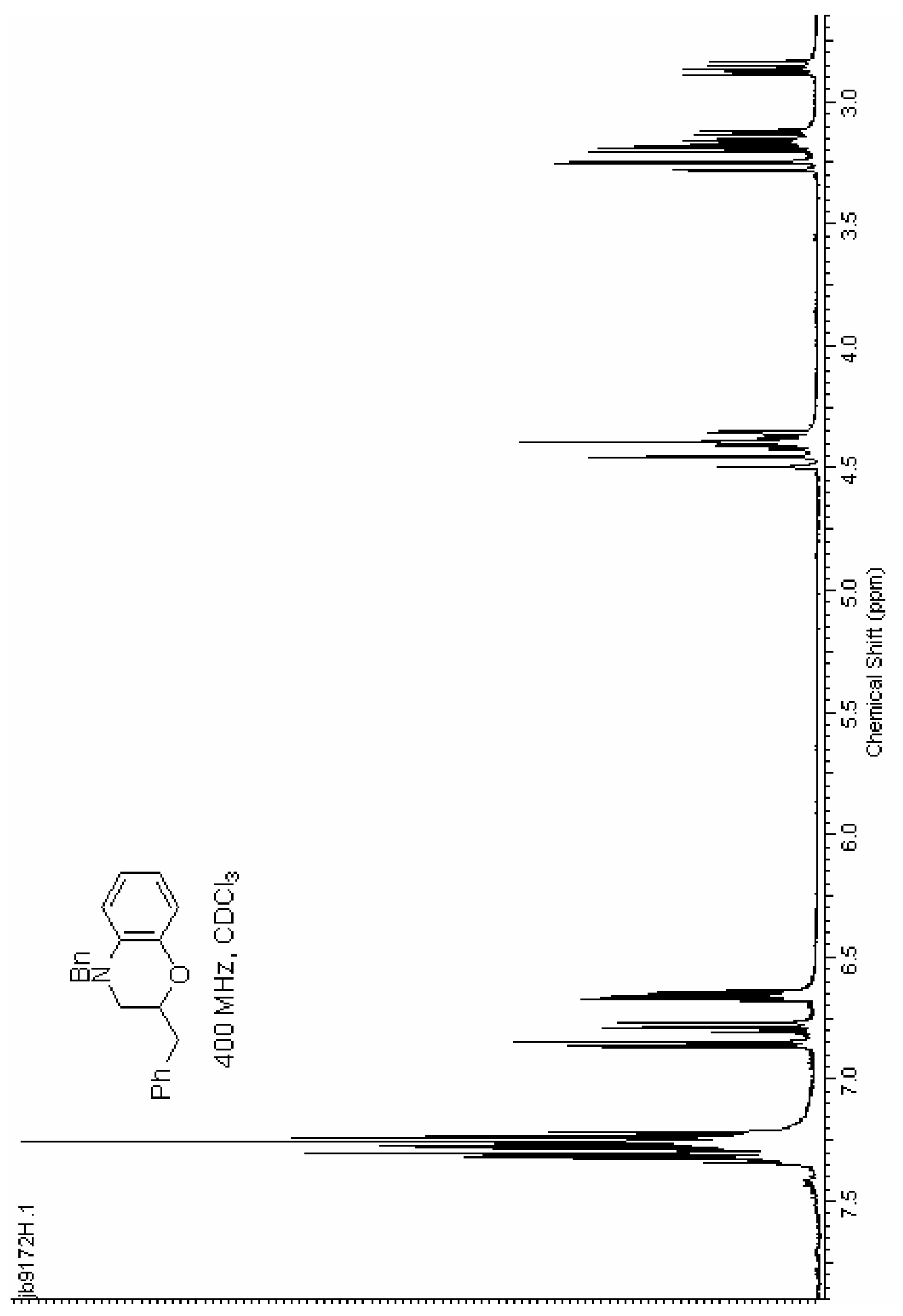




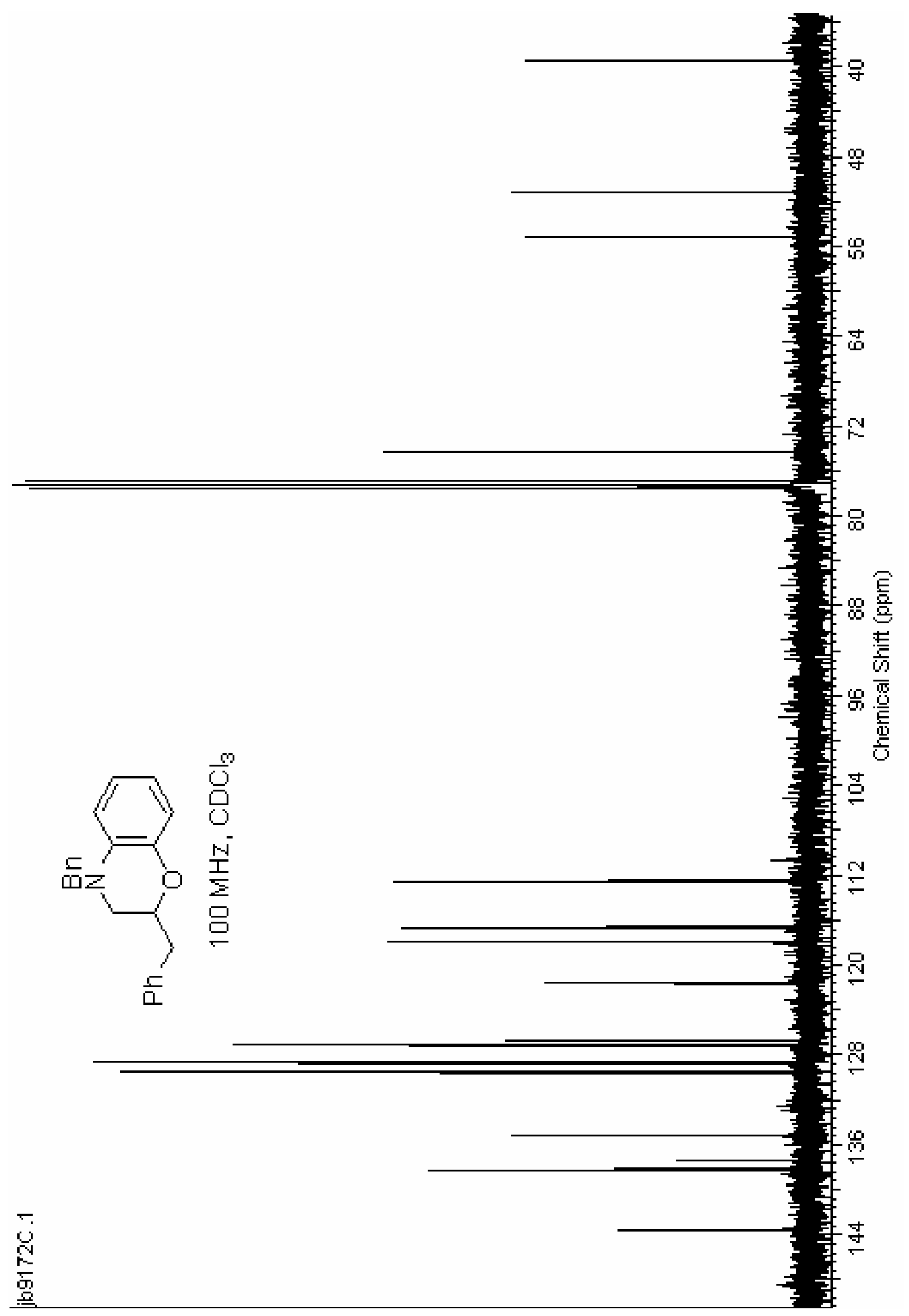




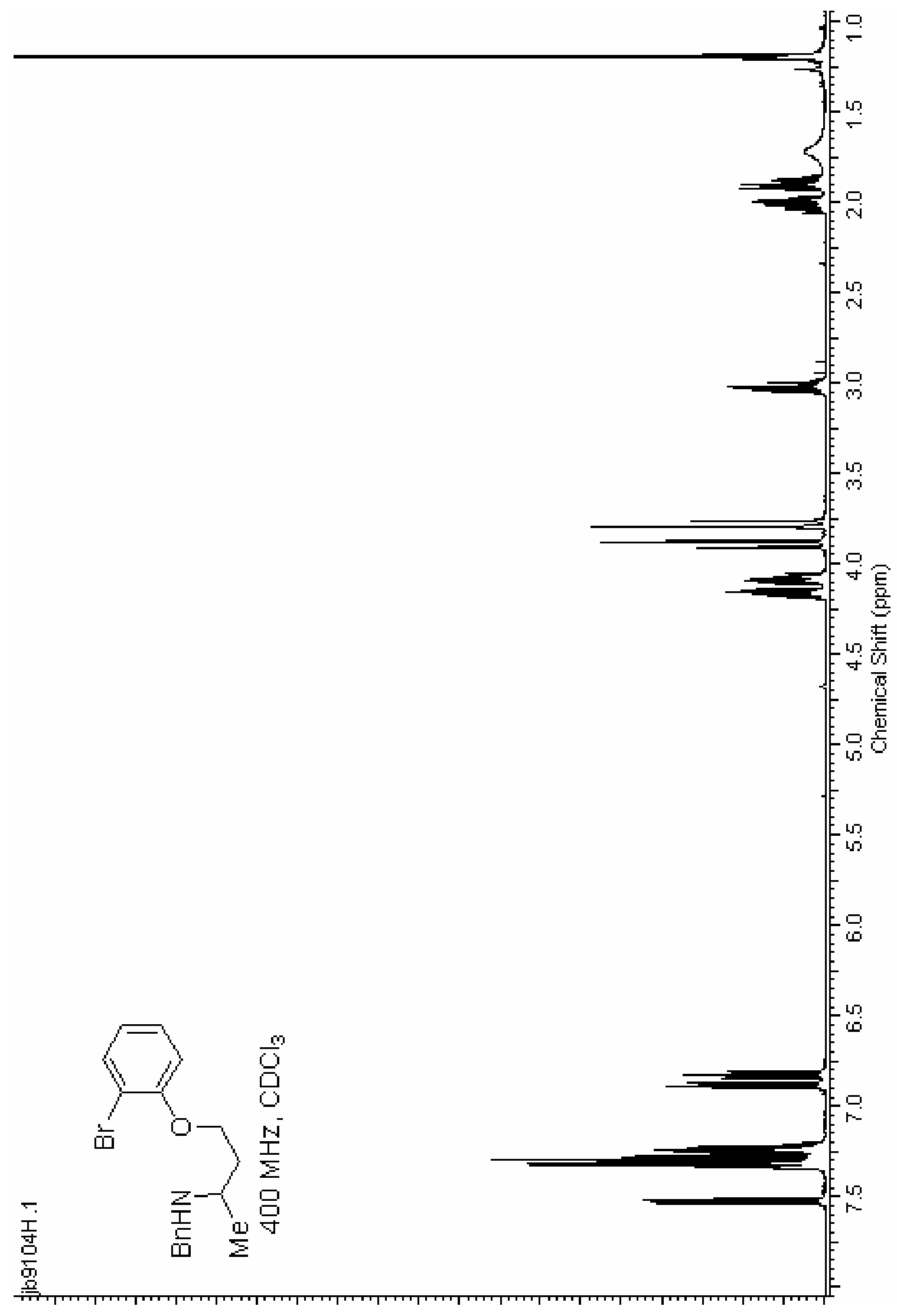




$$
1
$$




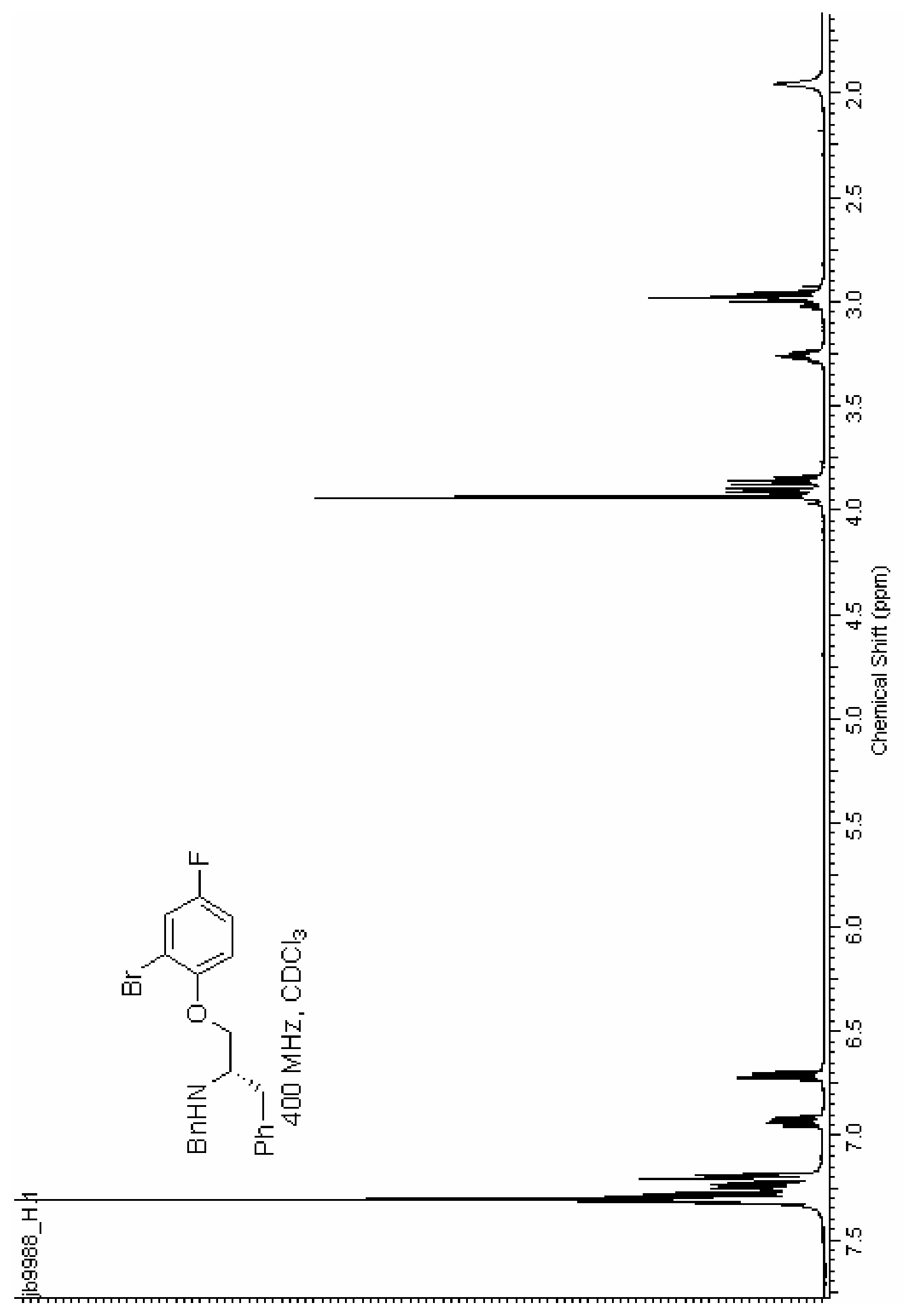




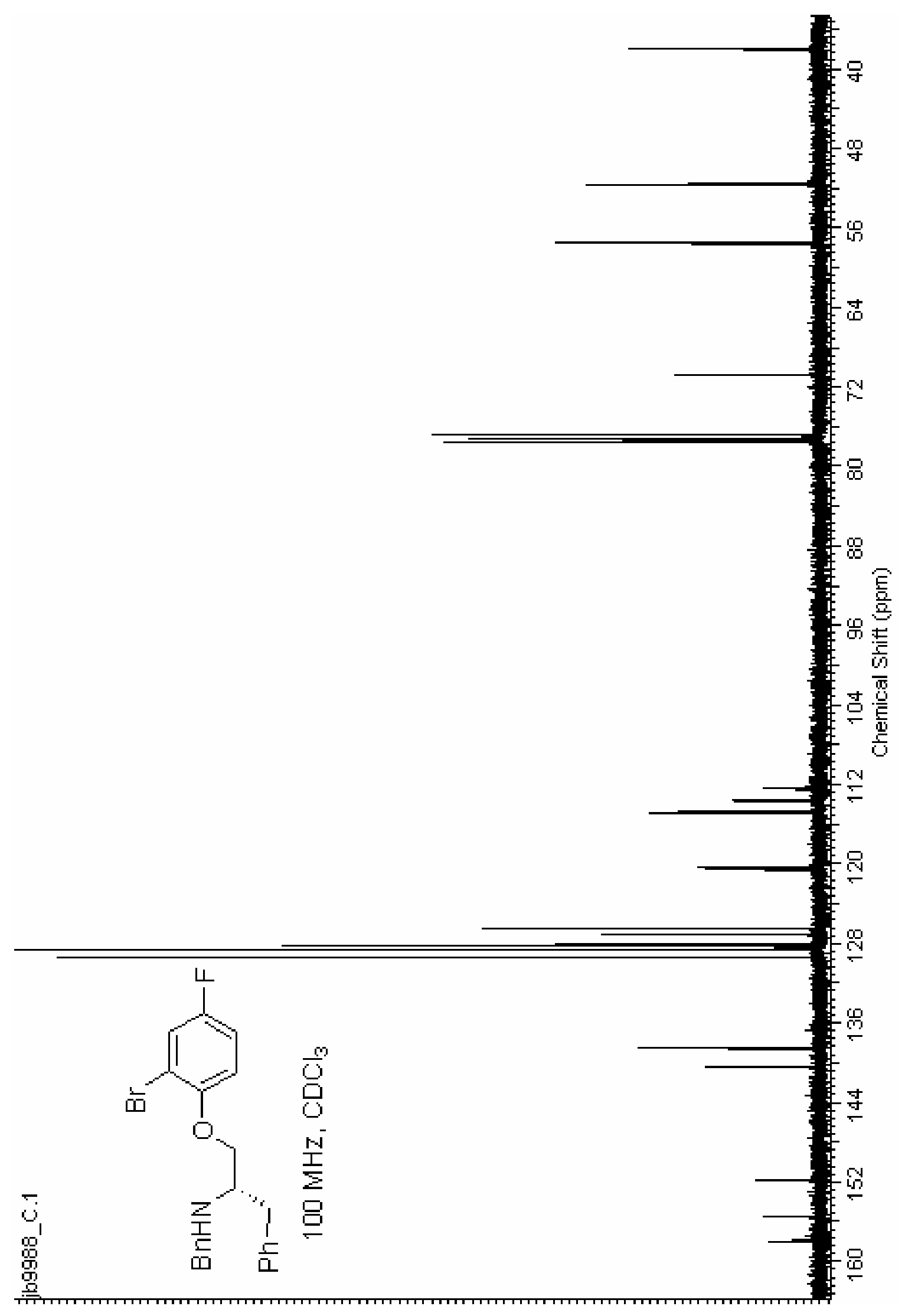




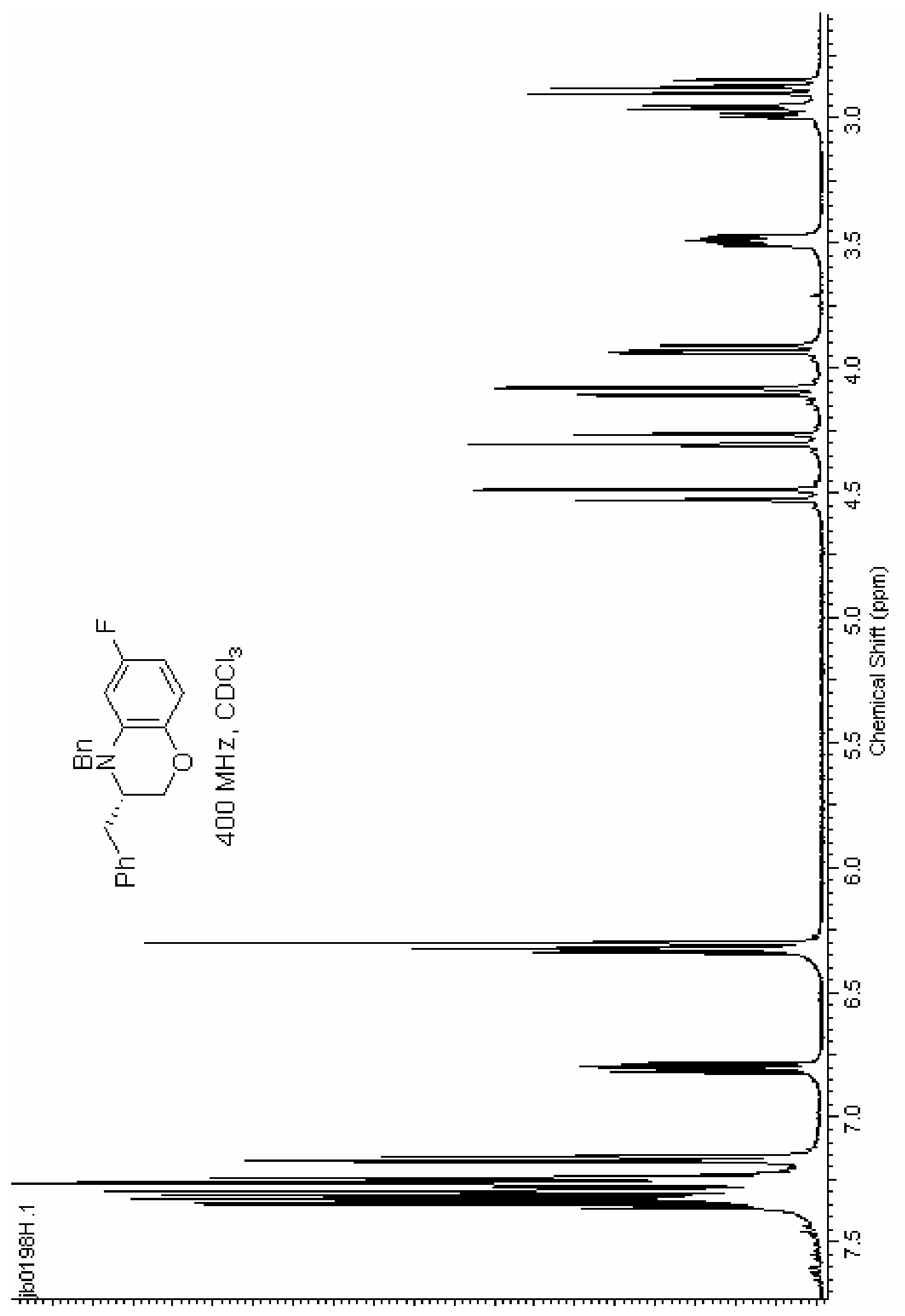




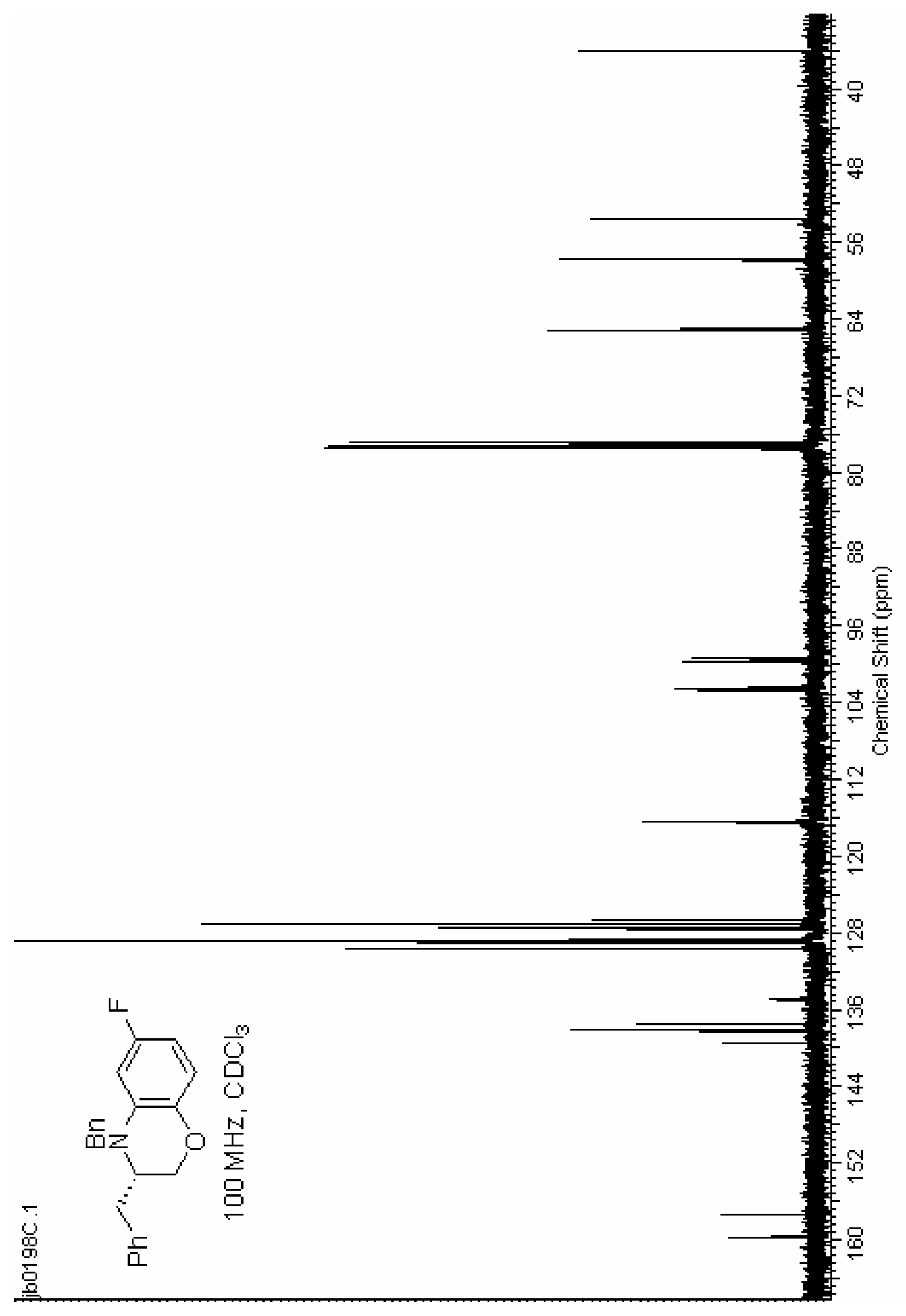




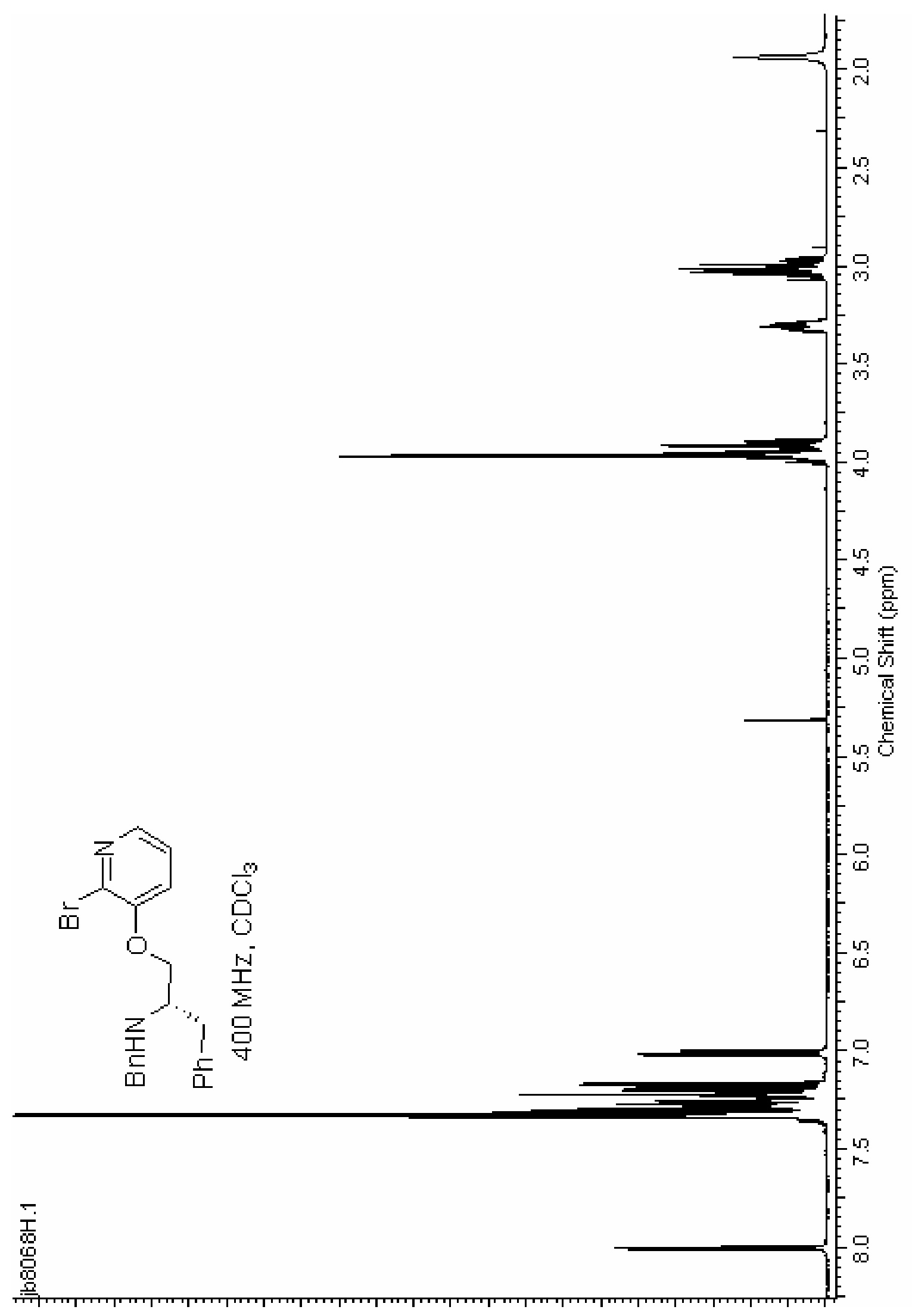




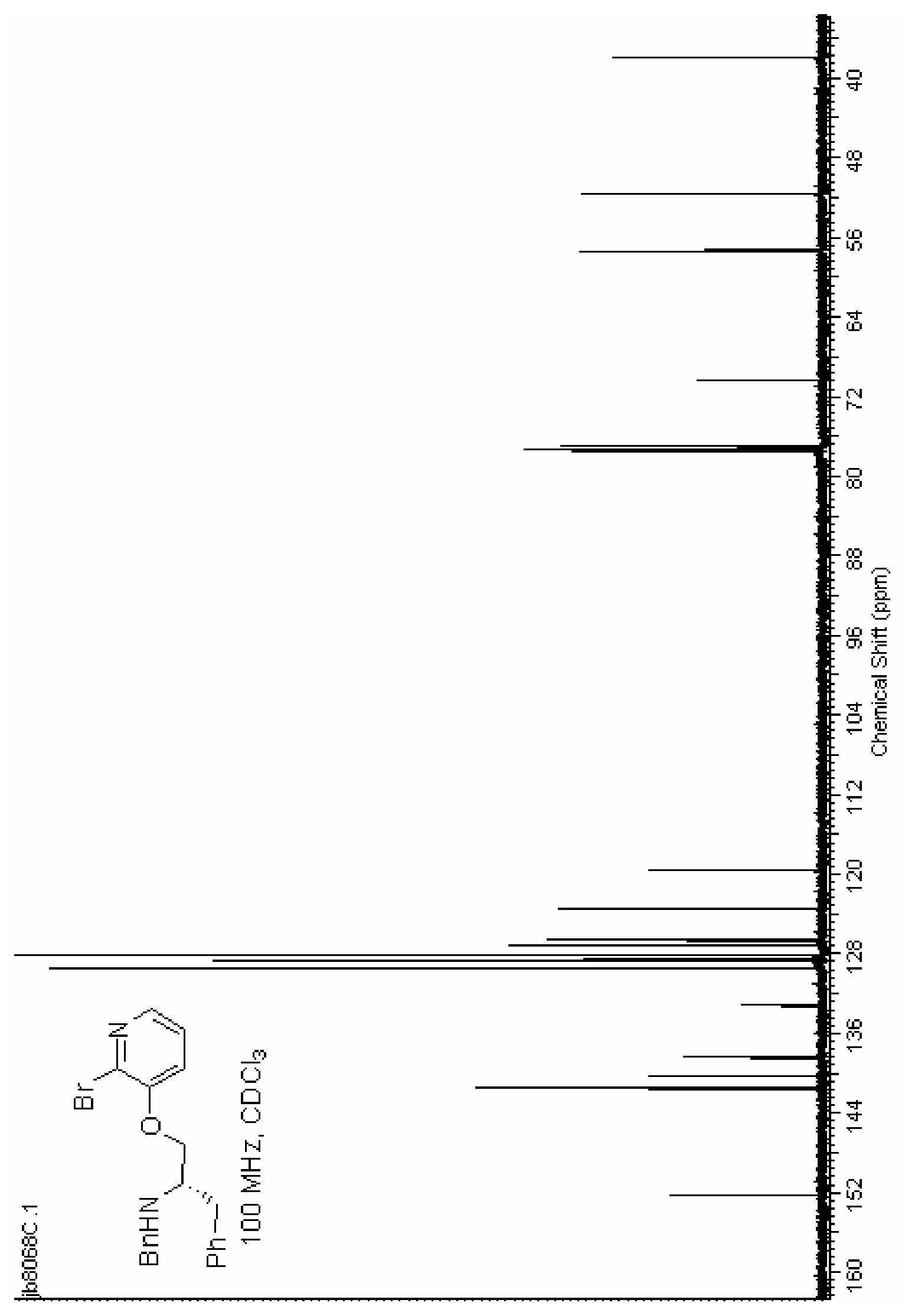




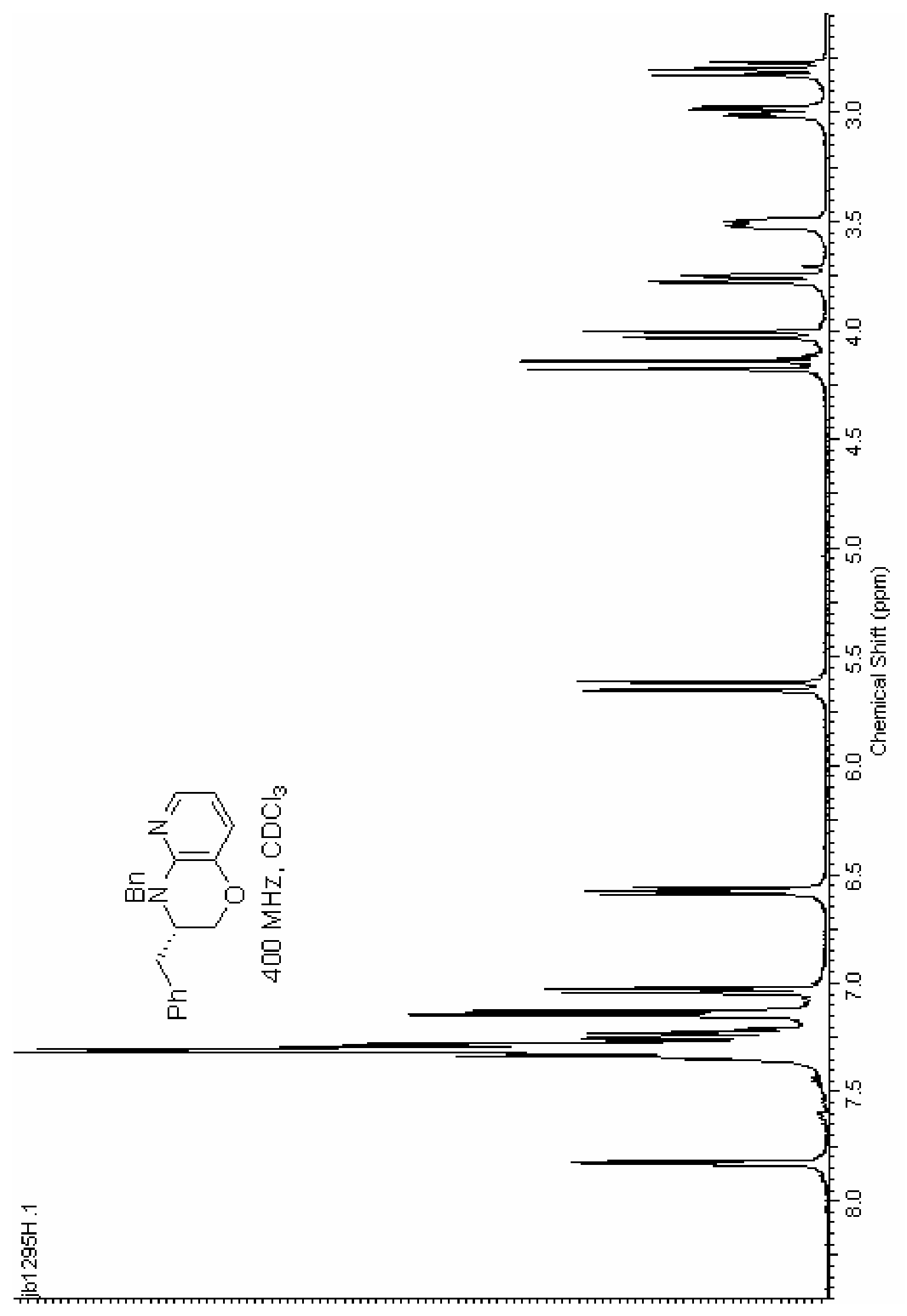




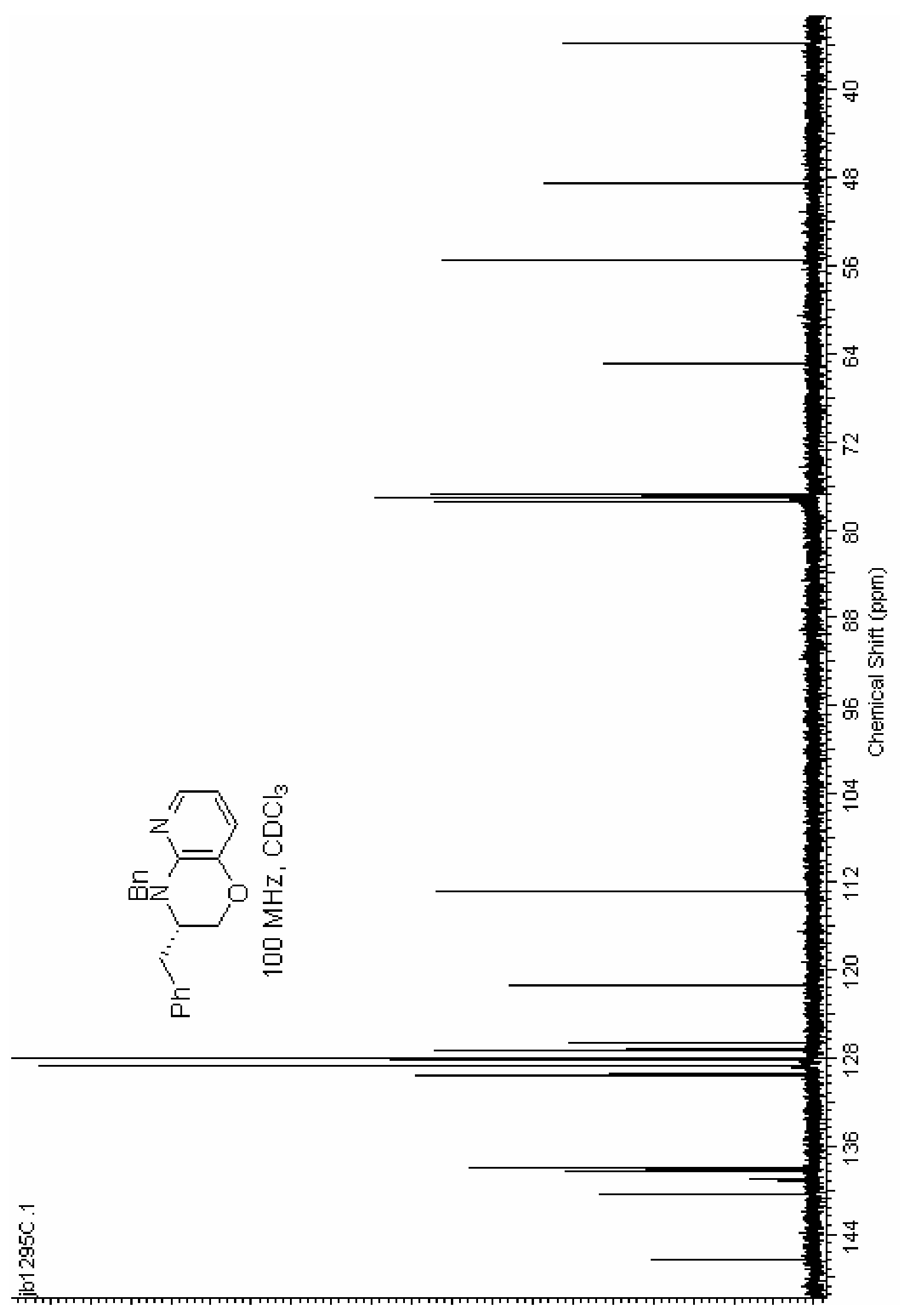




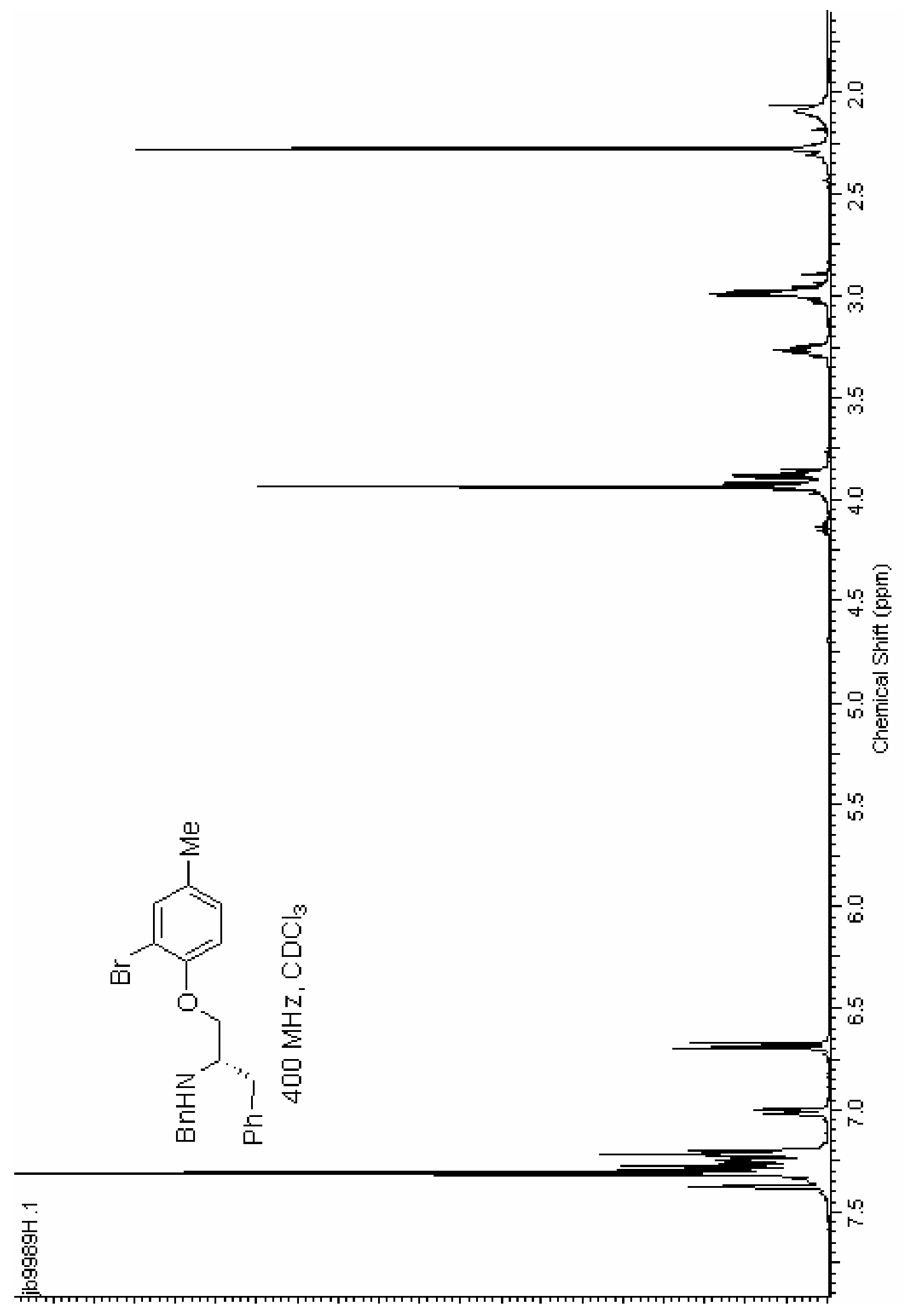




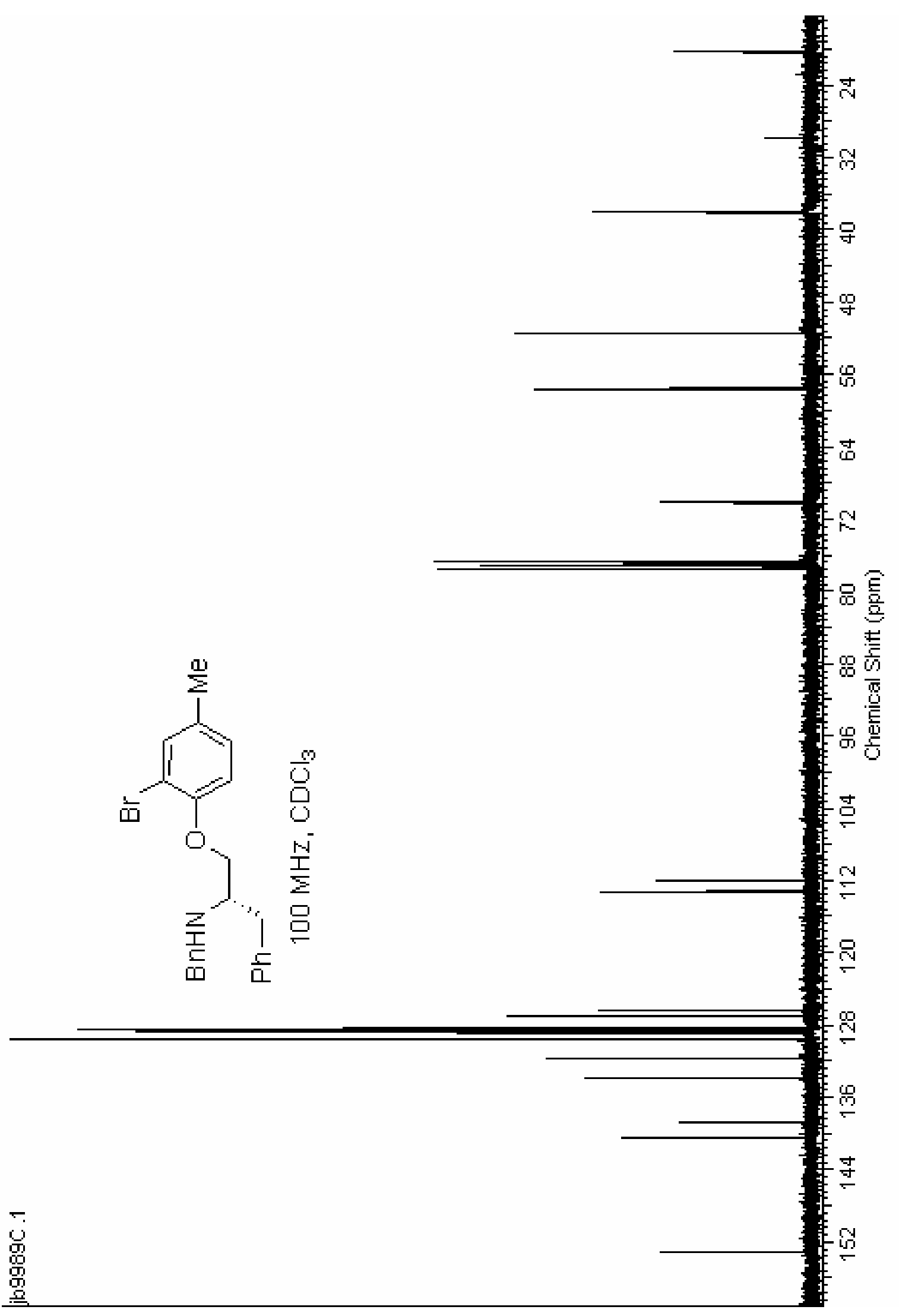




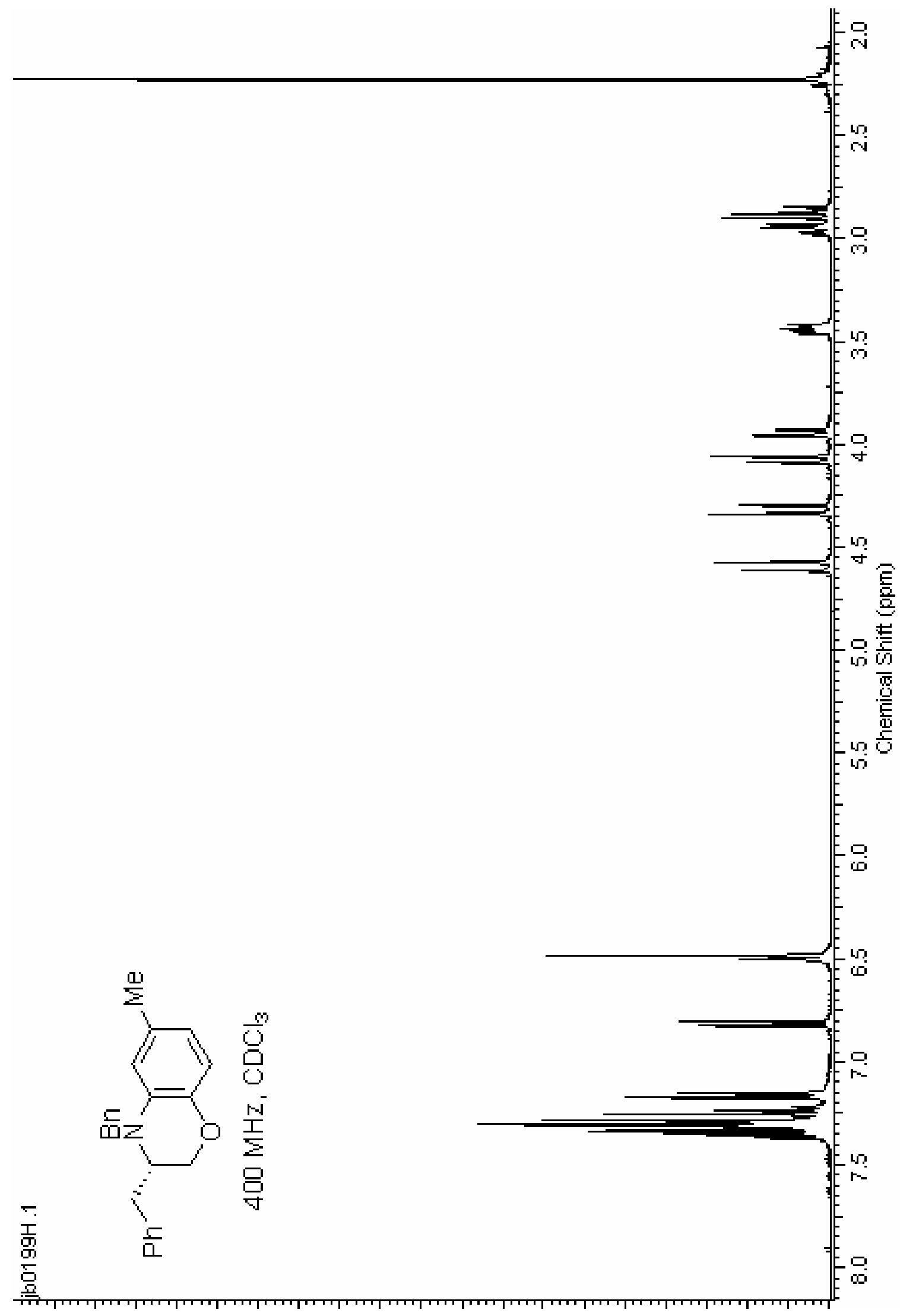




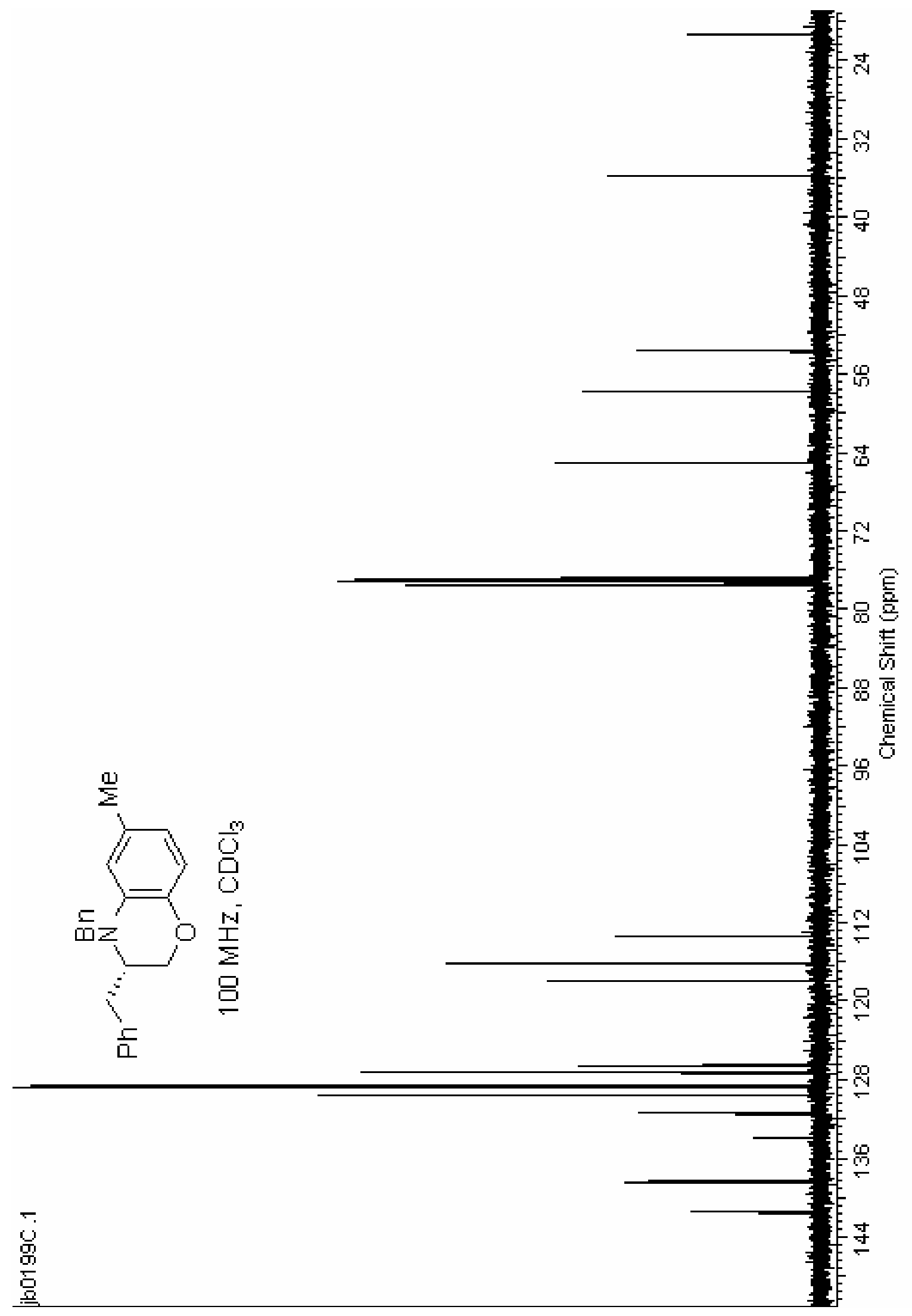




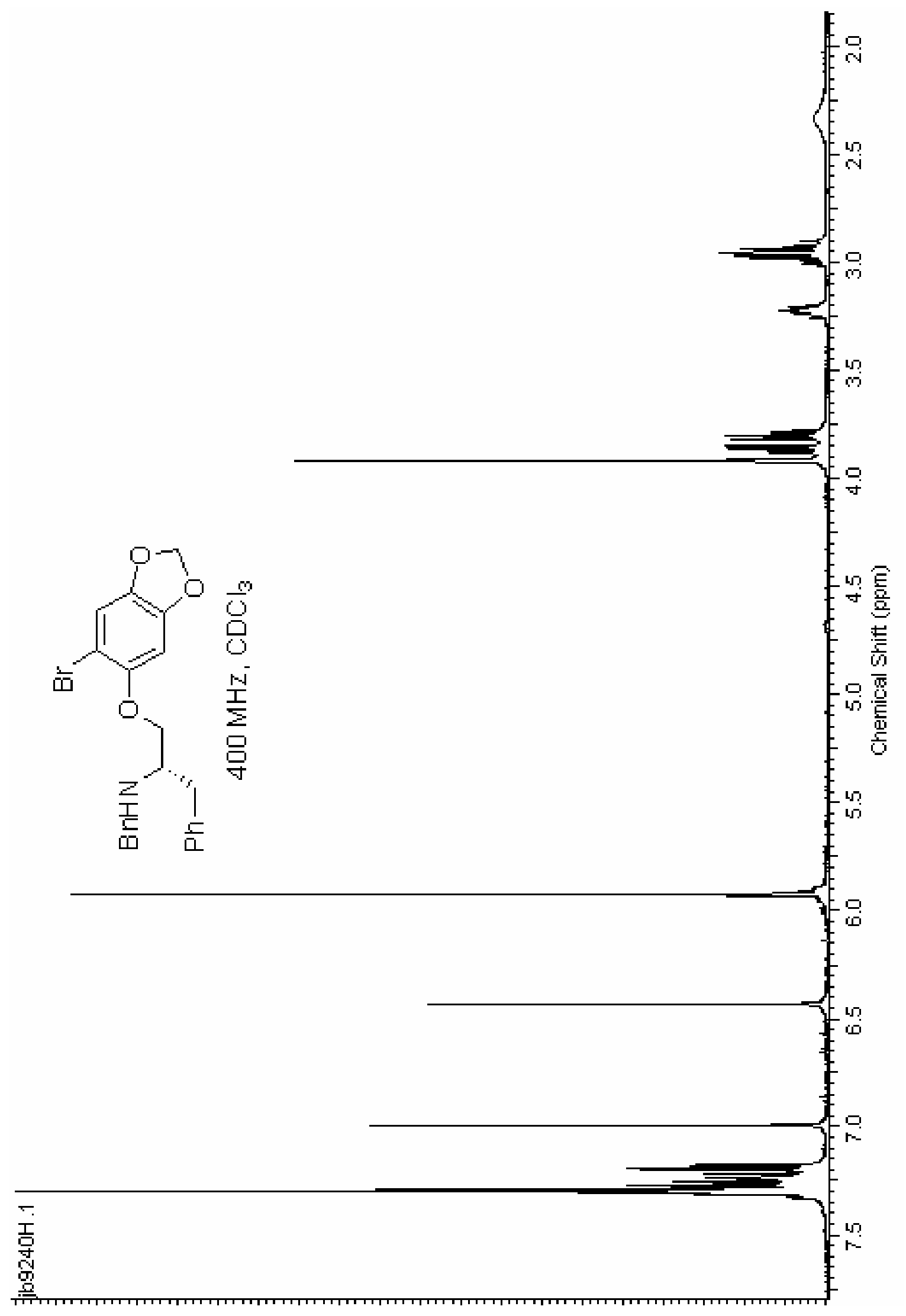




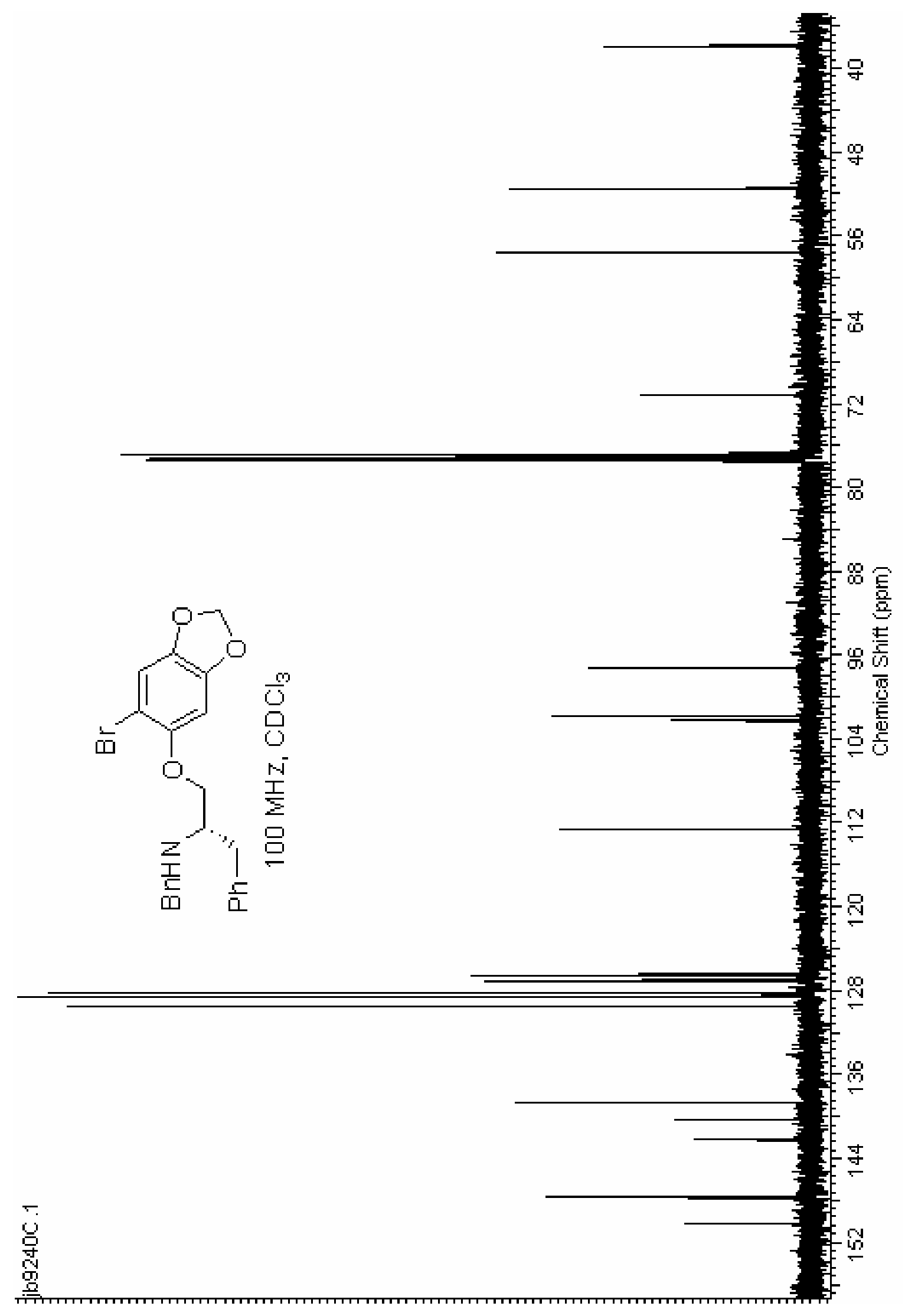




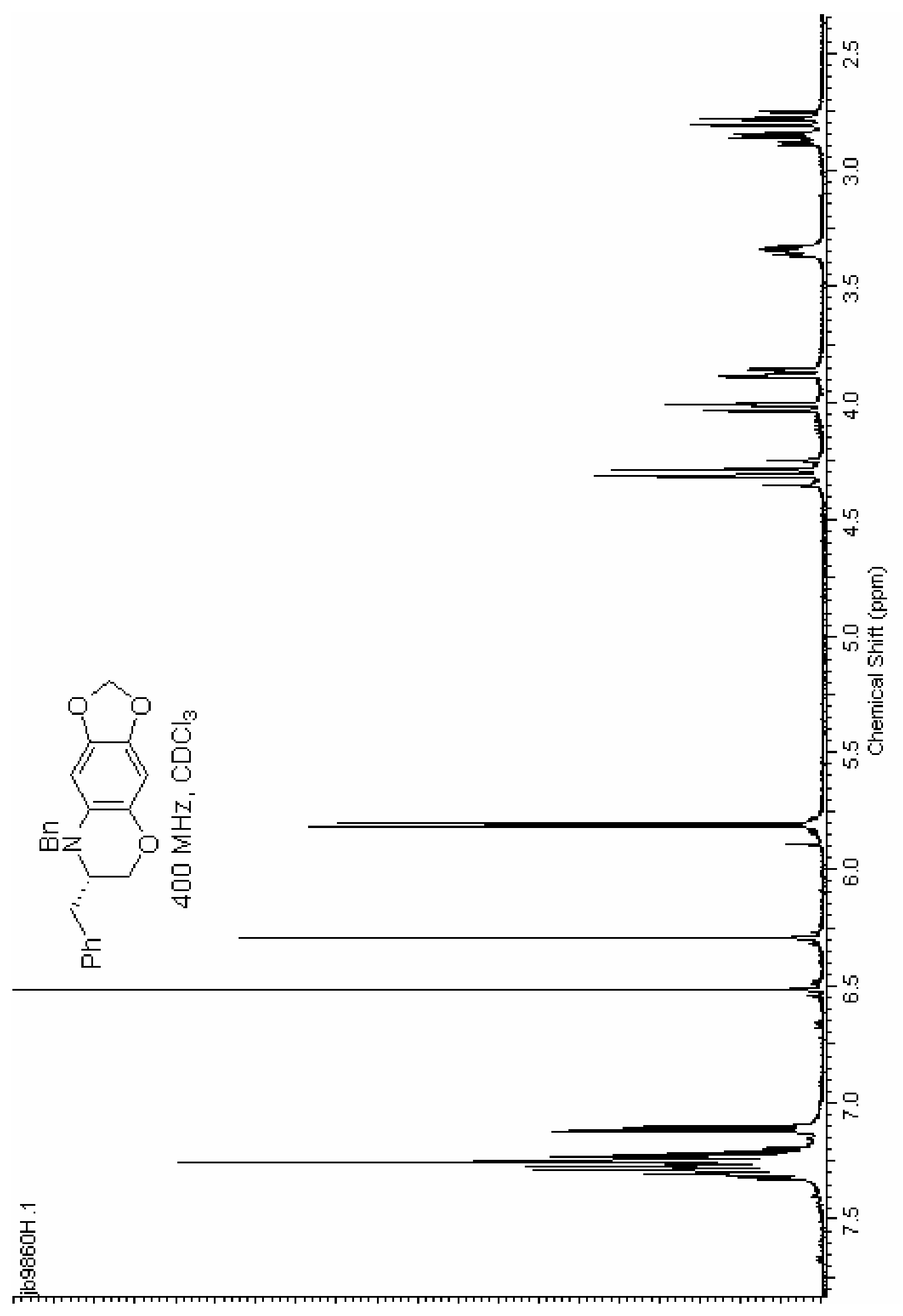




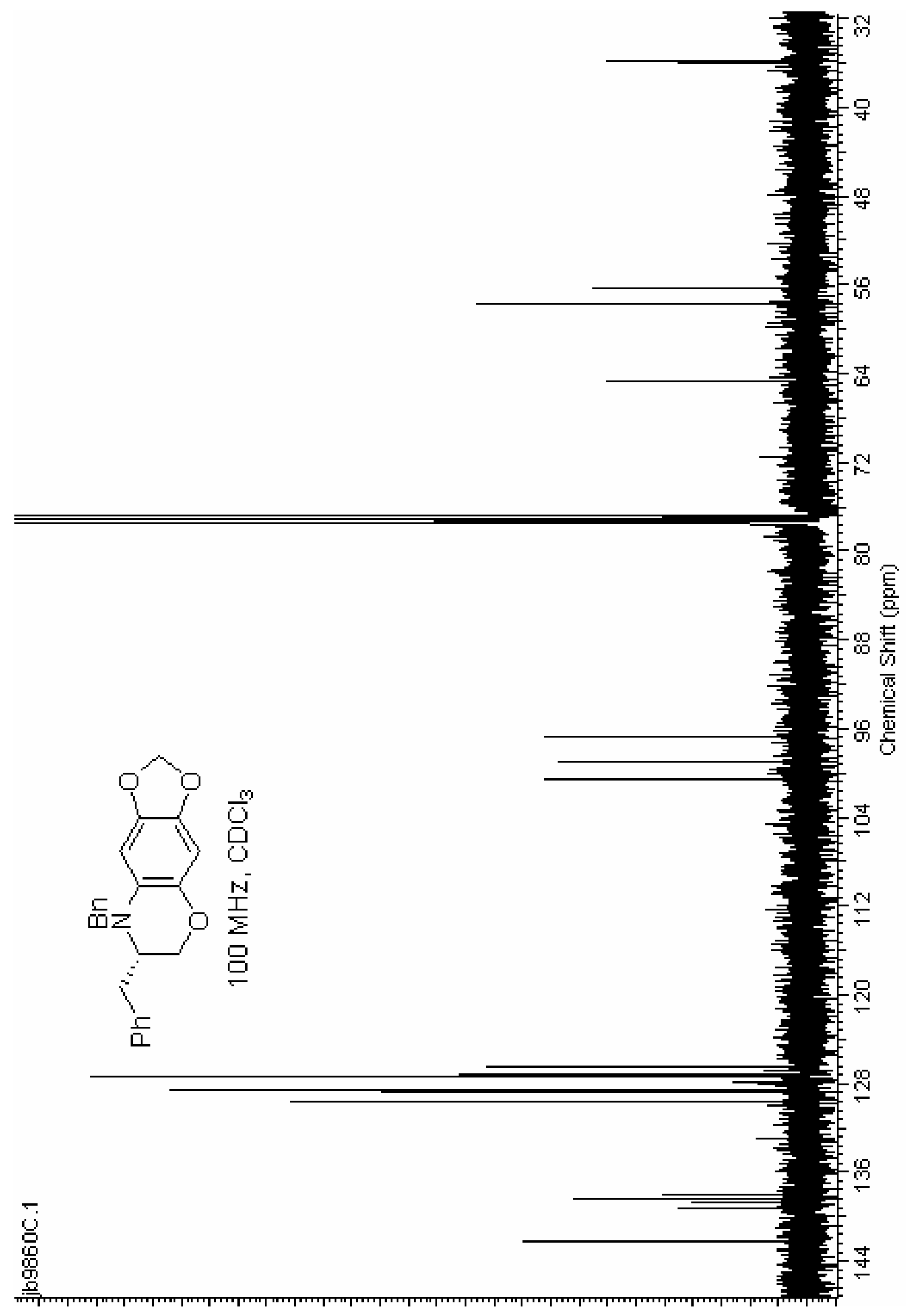




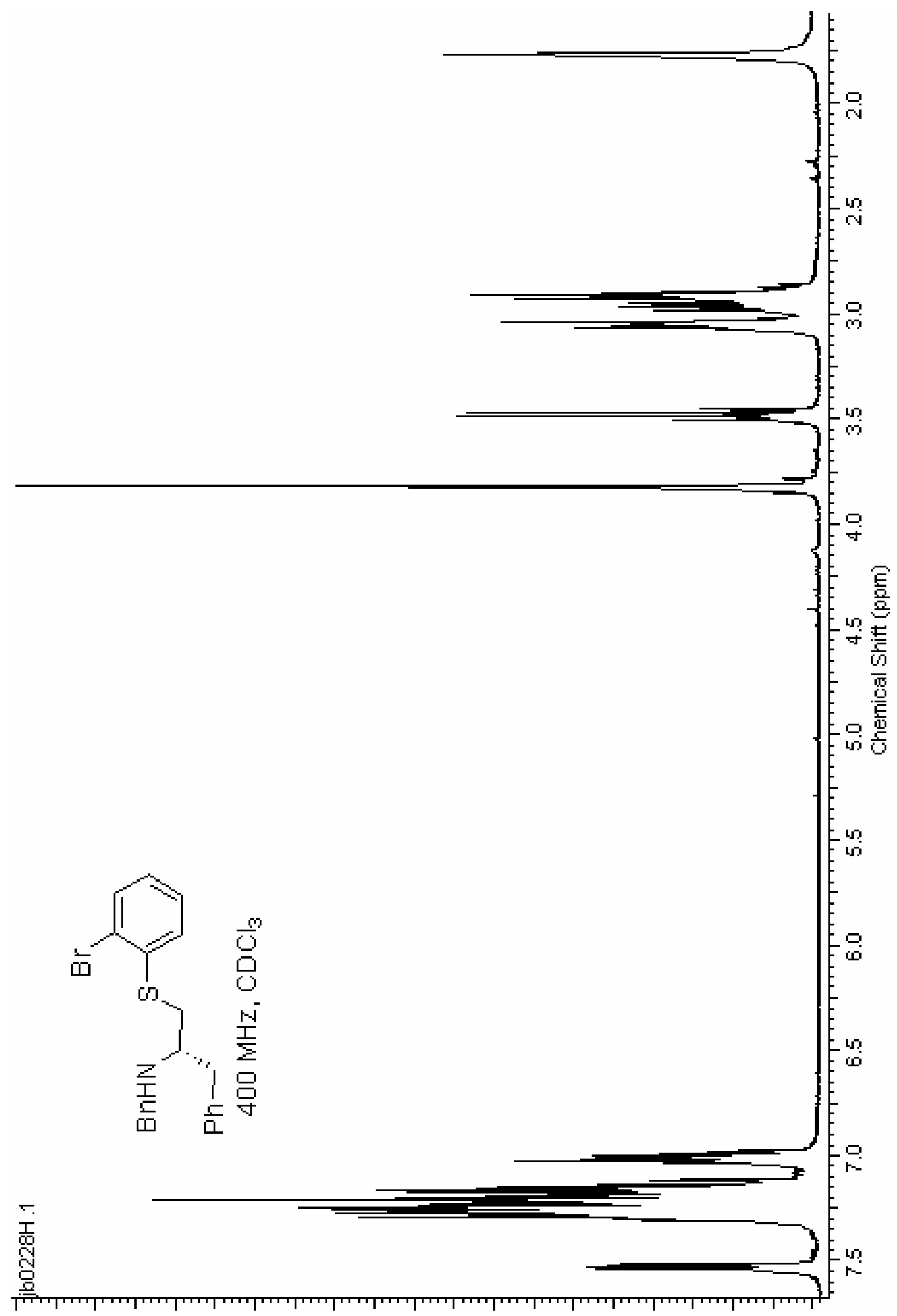




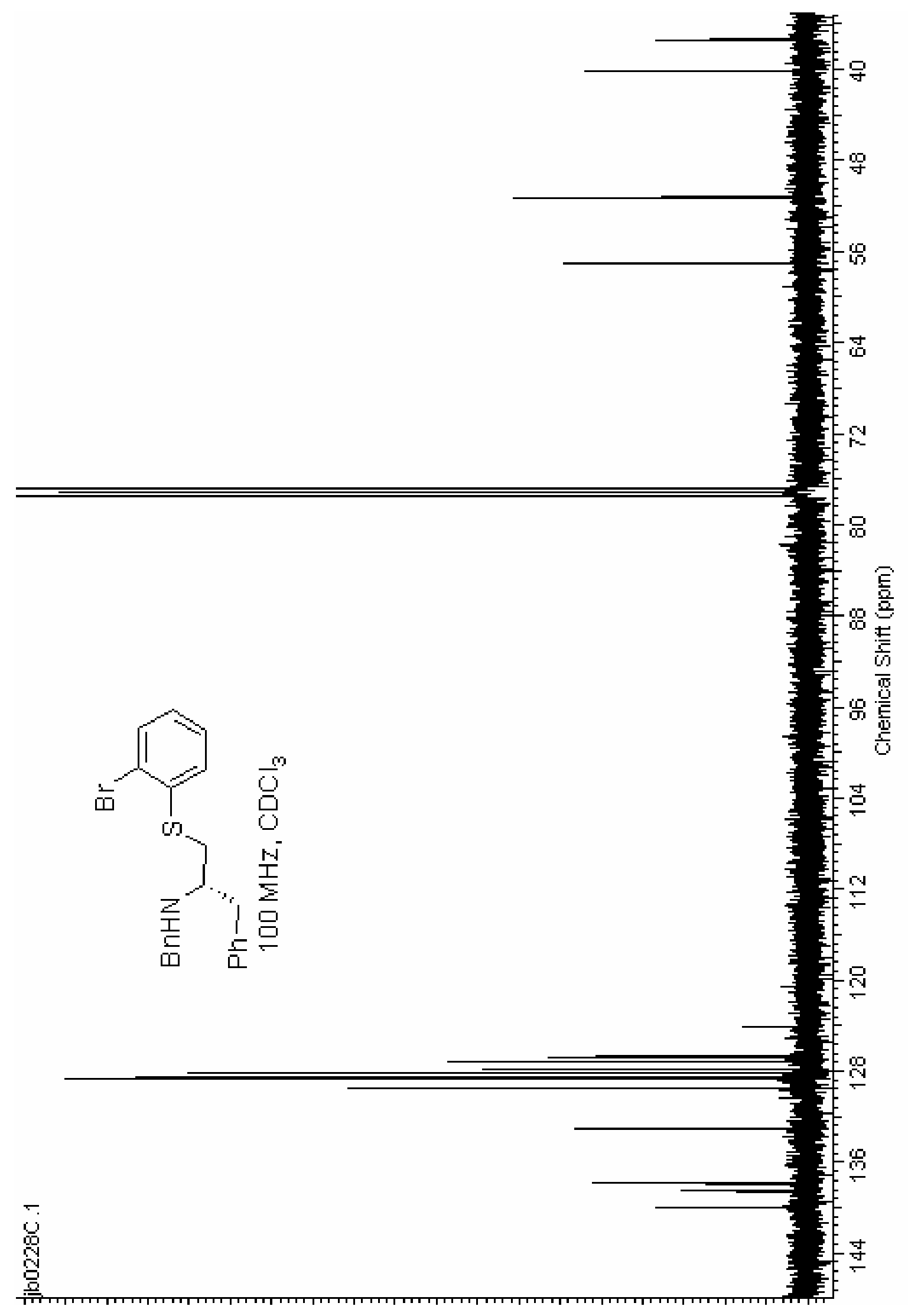




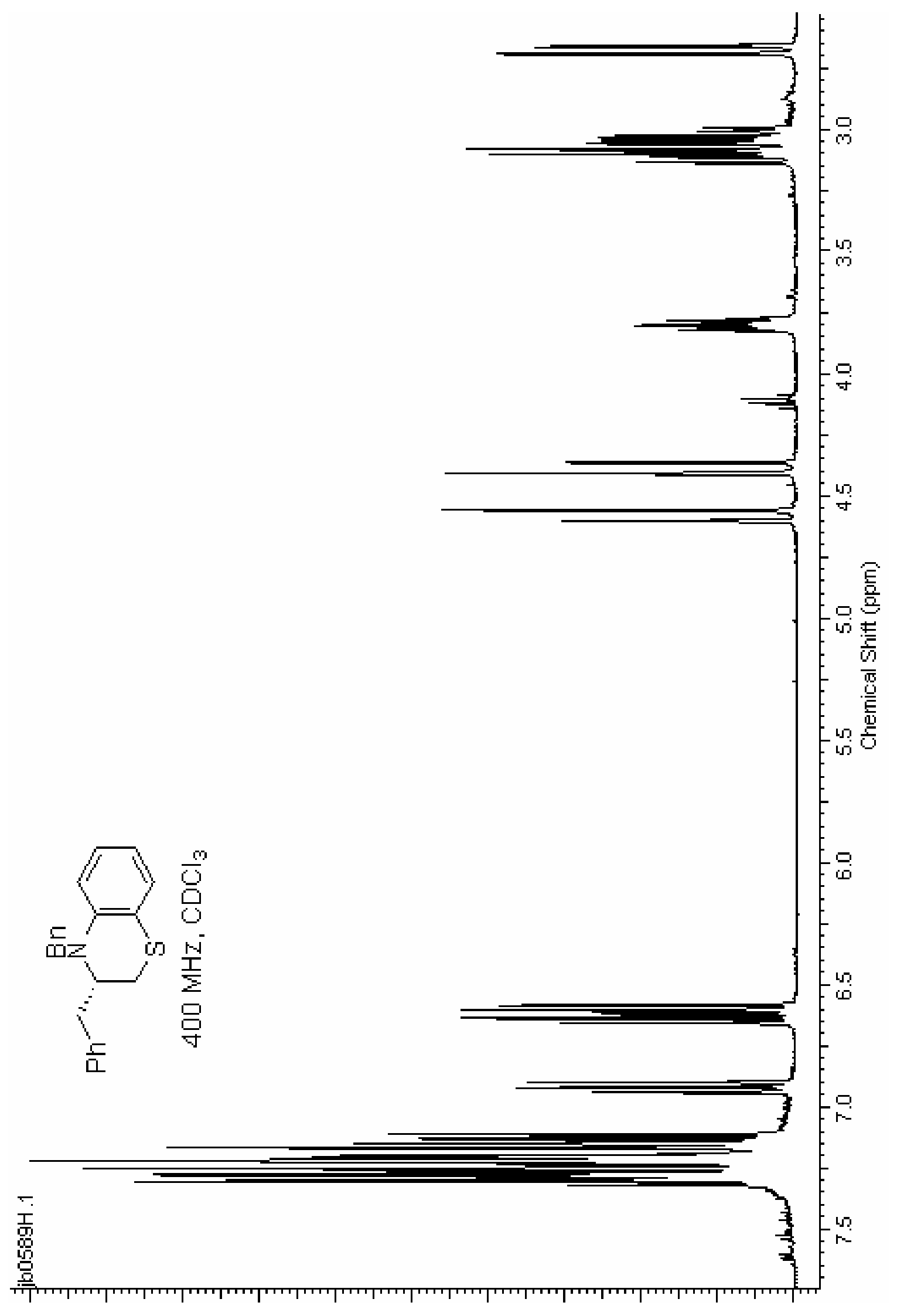




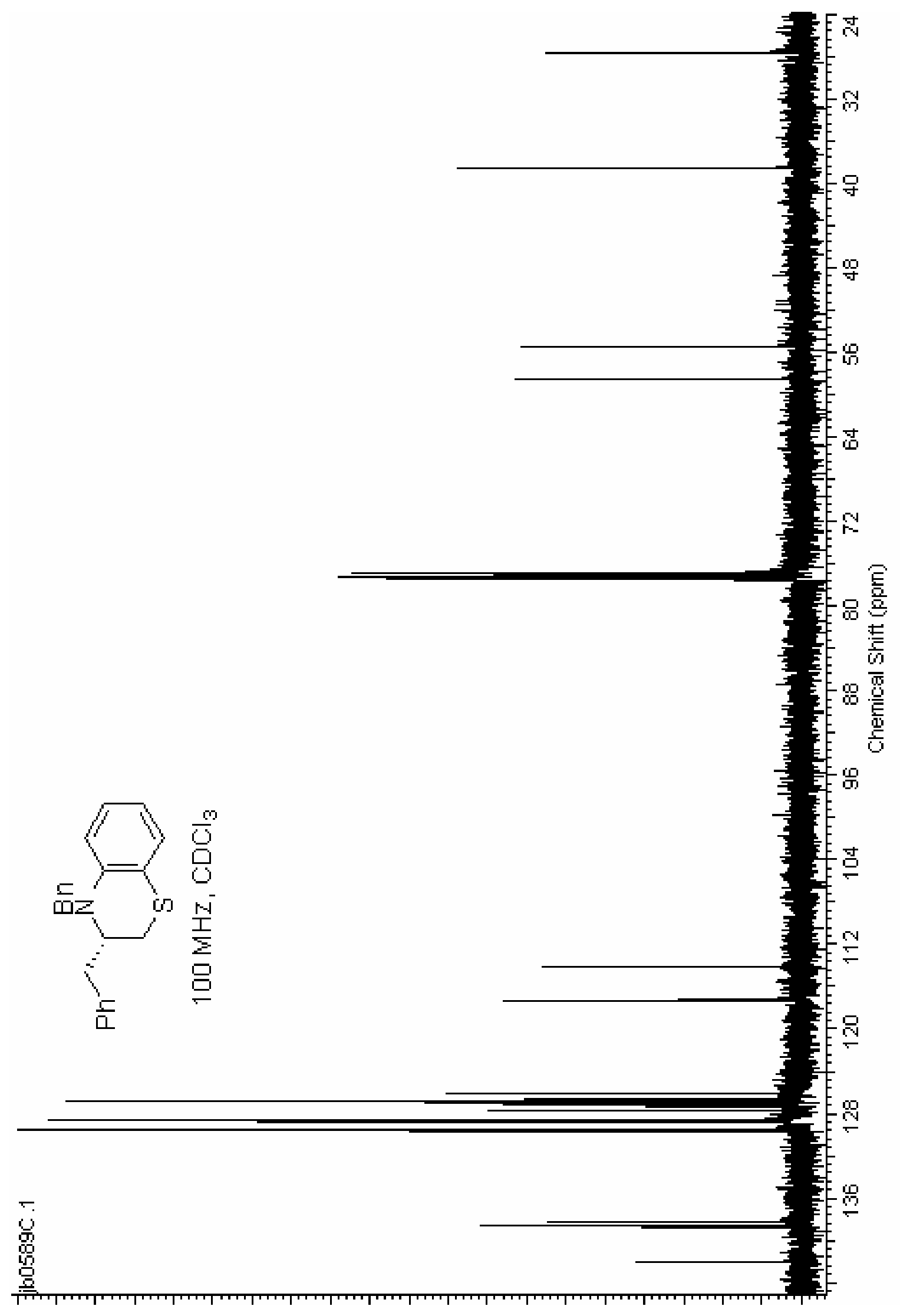




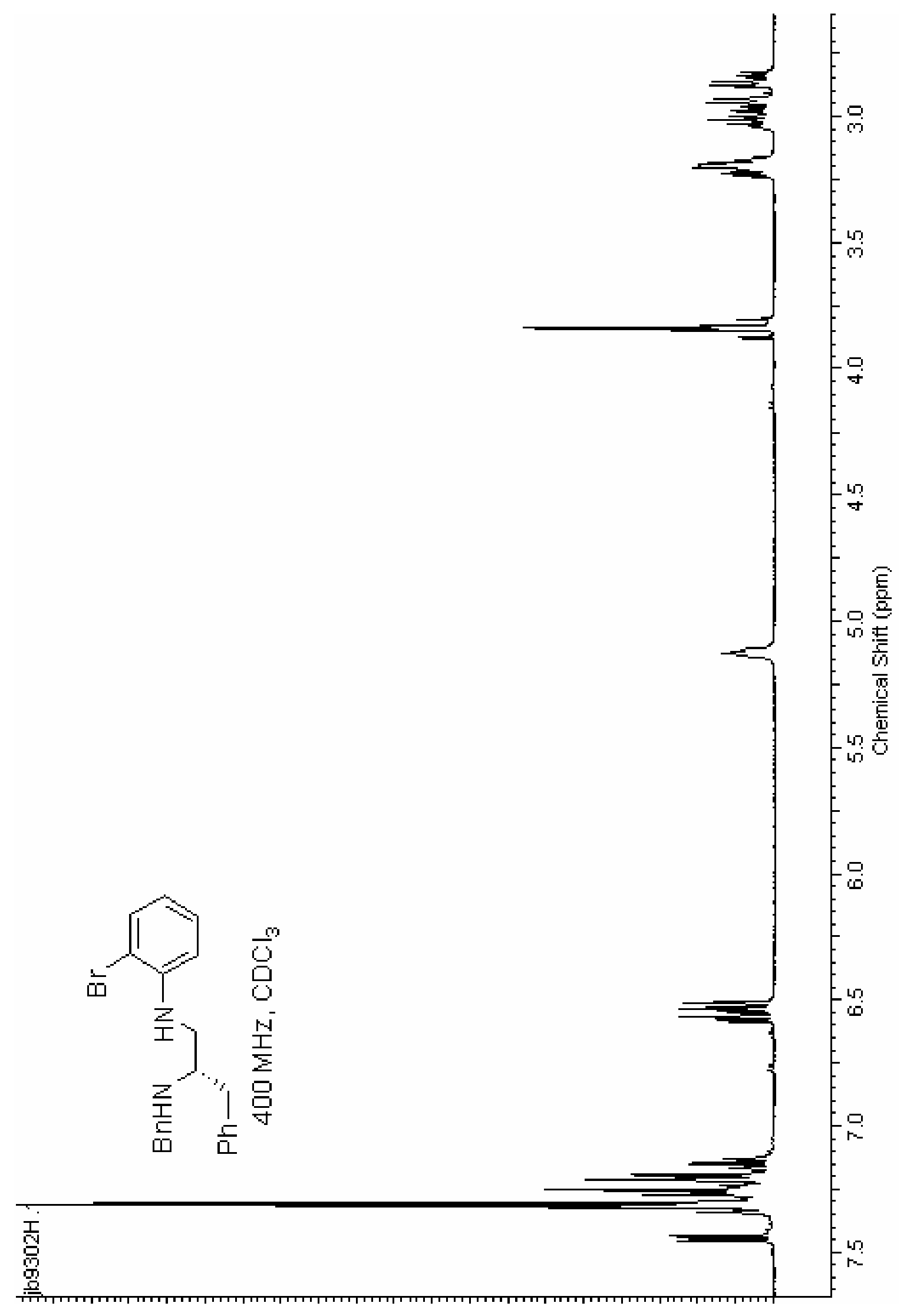




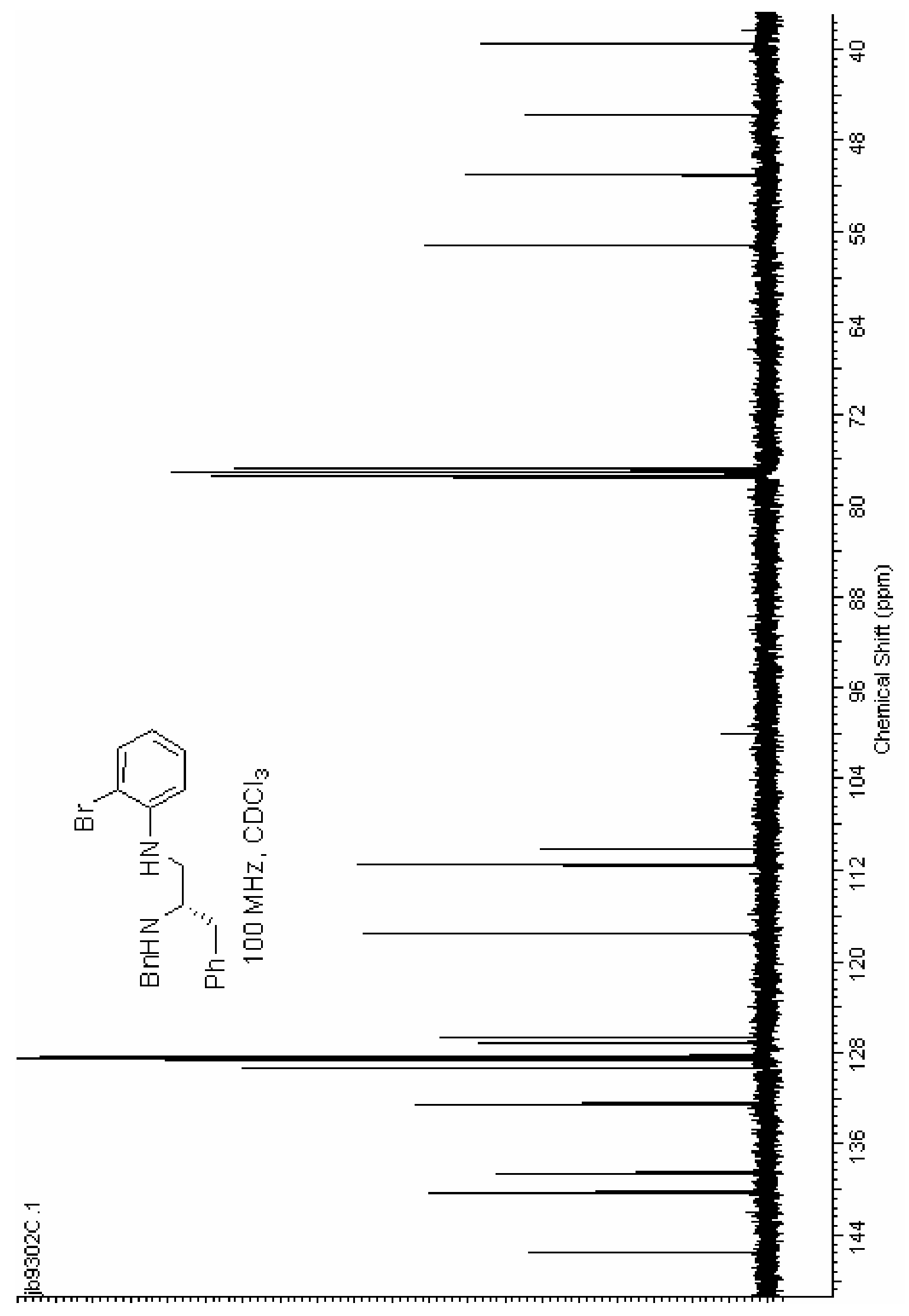




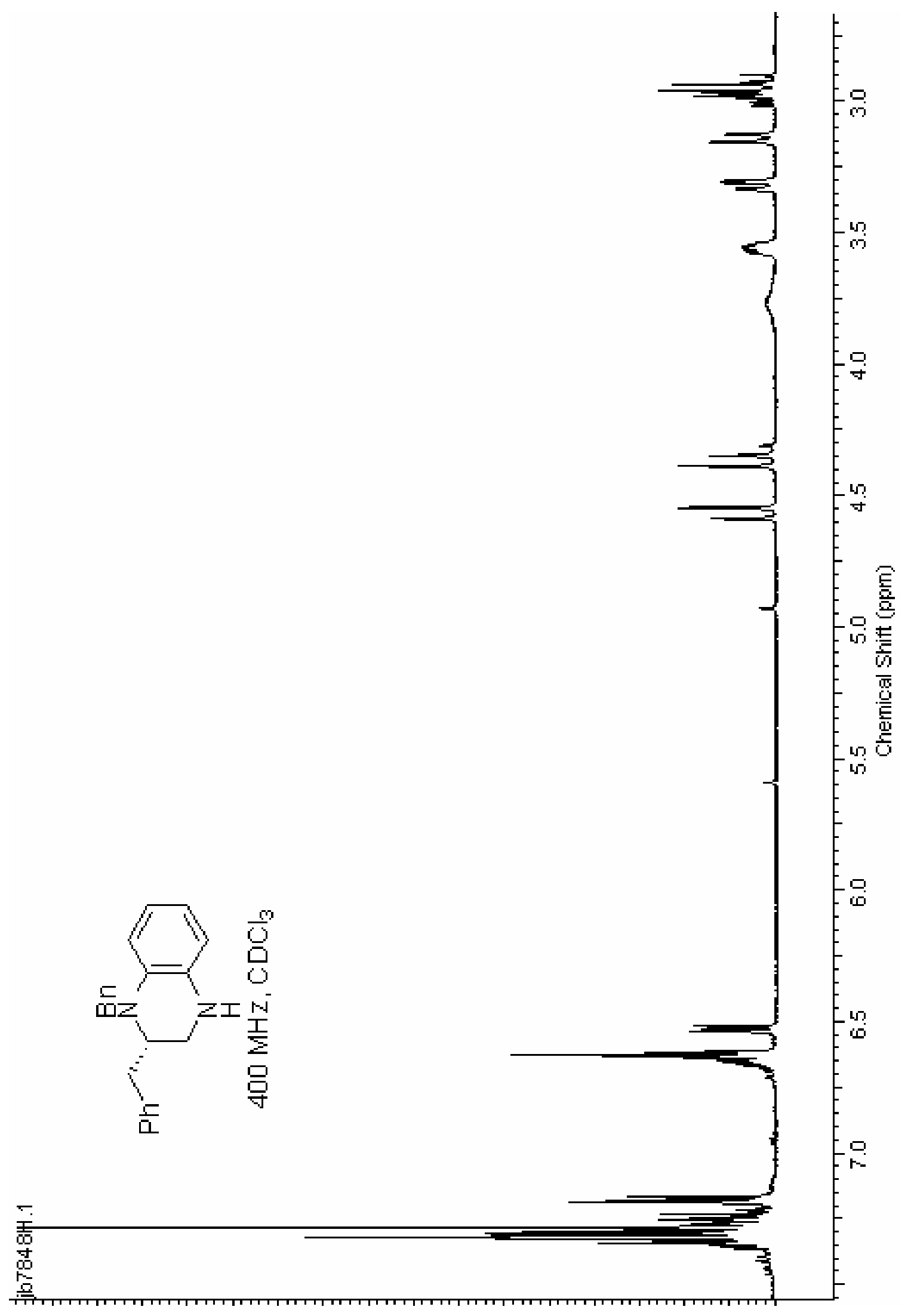




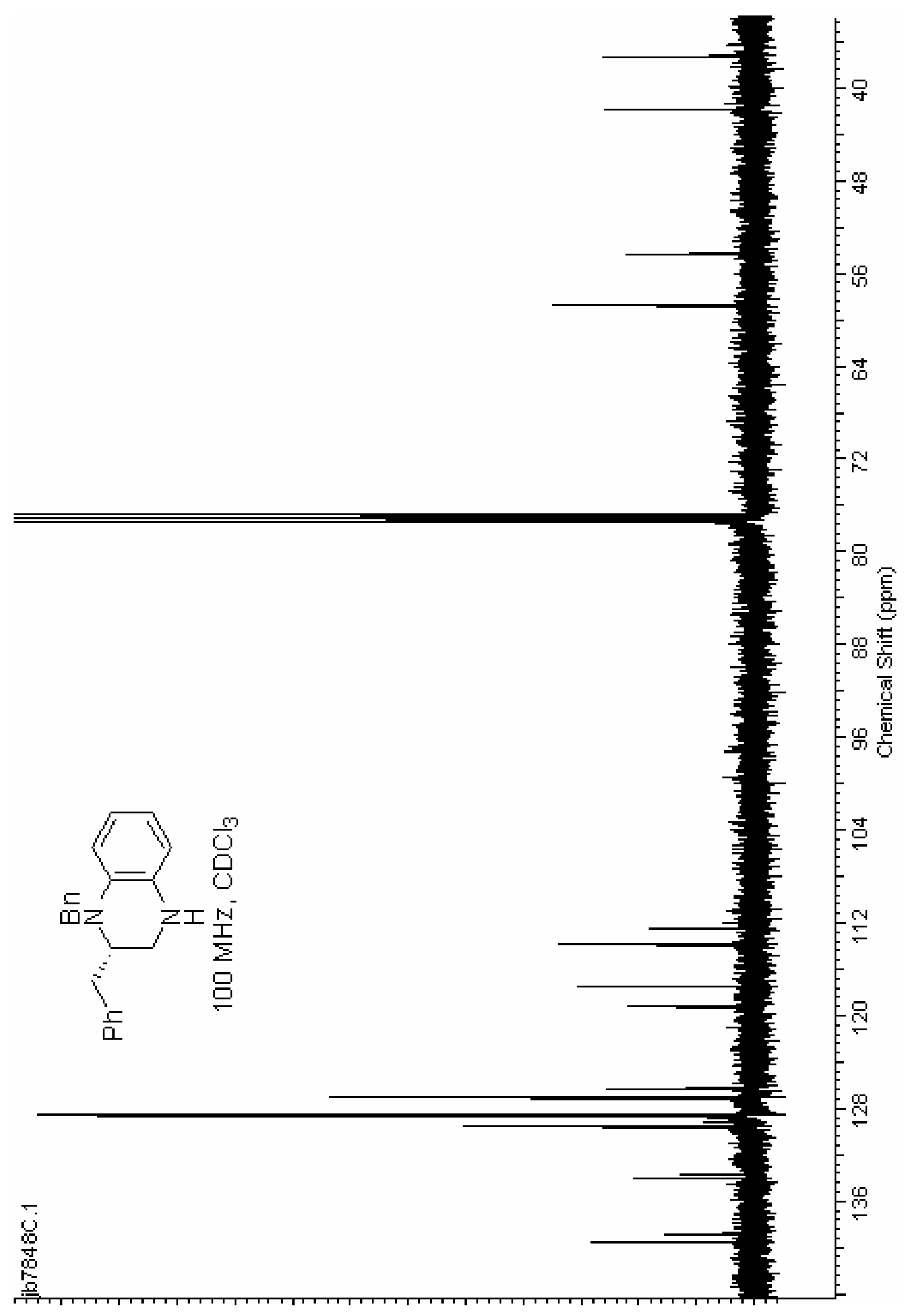




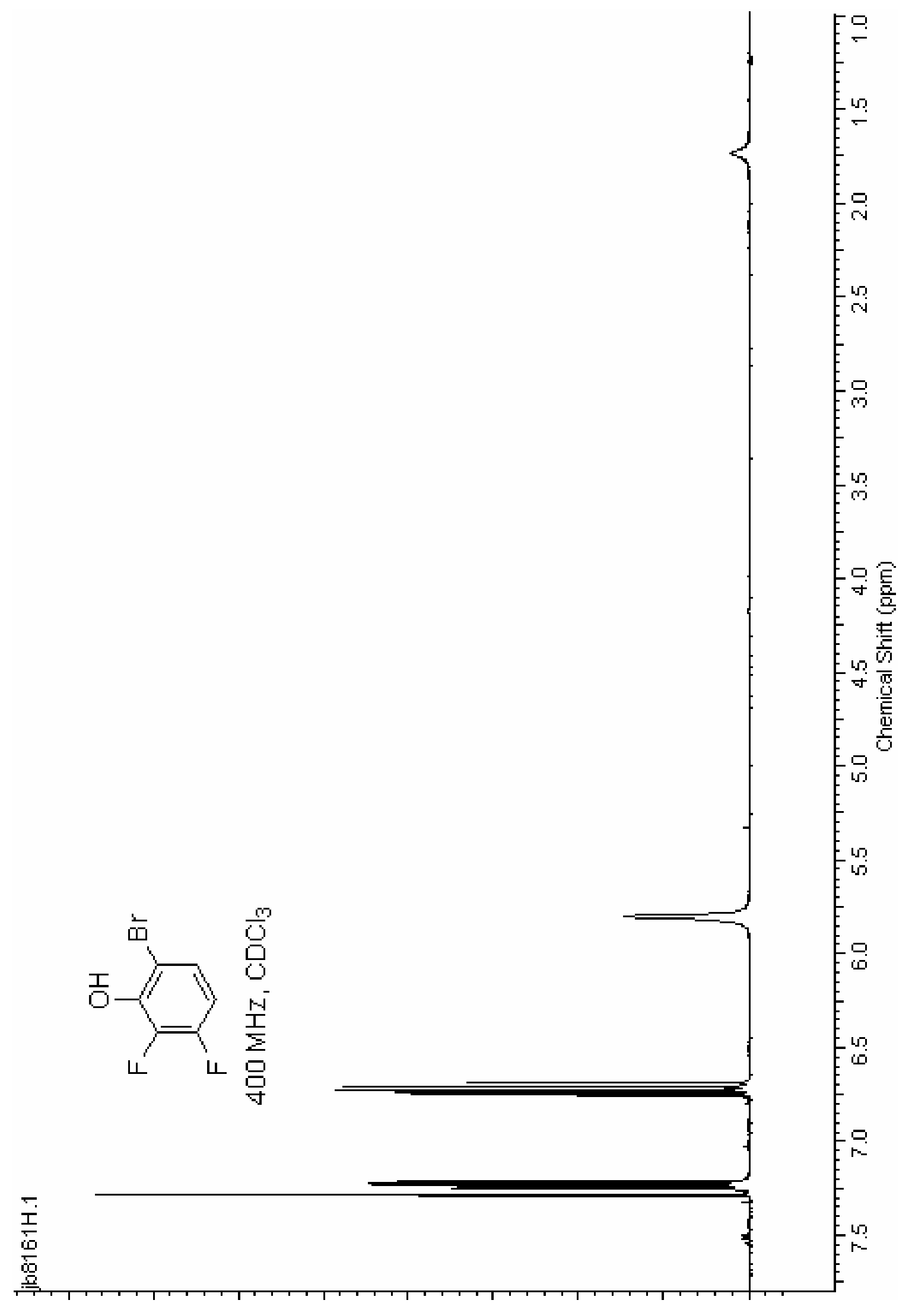




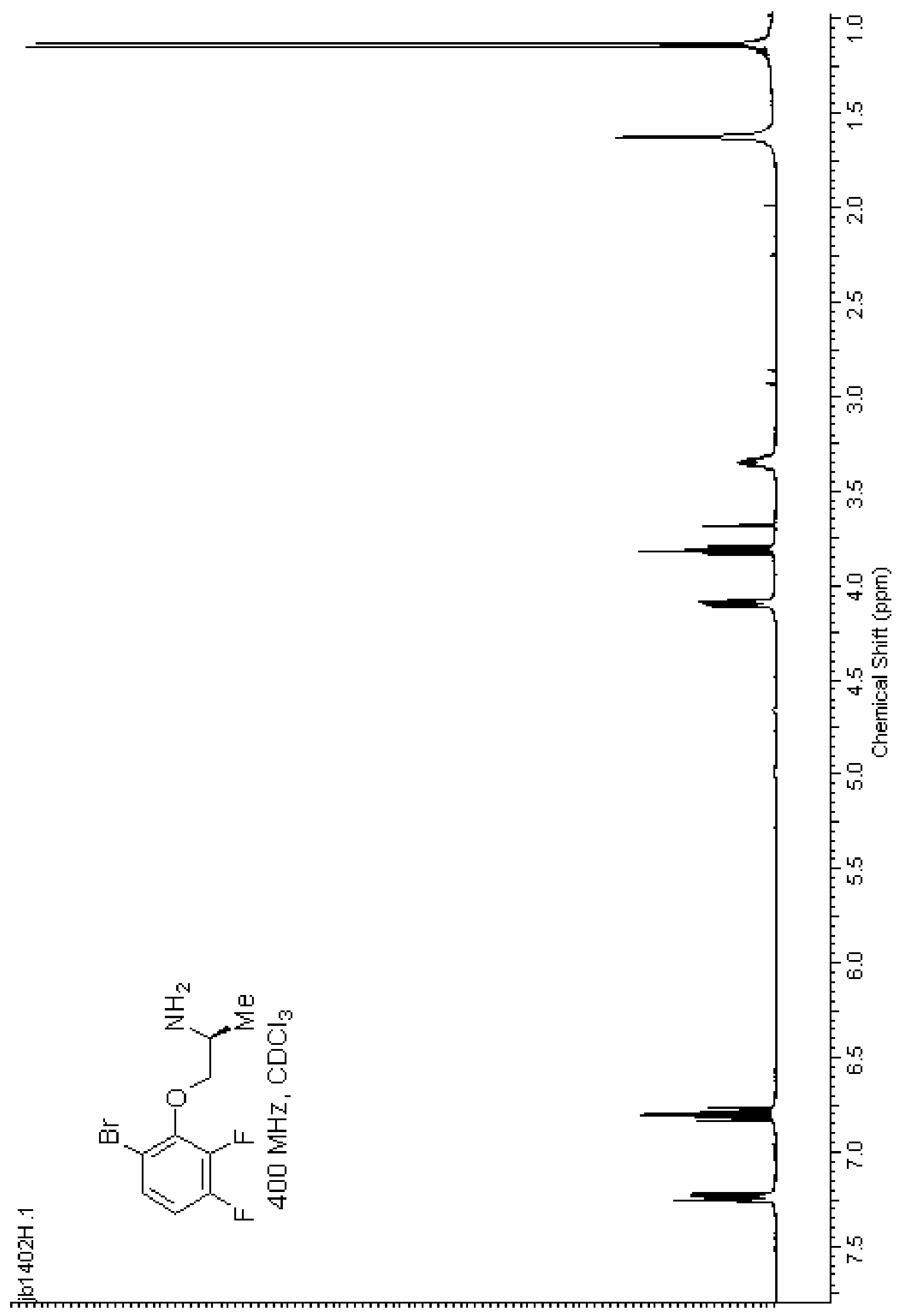




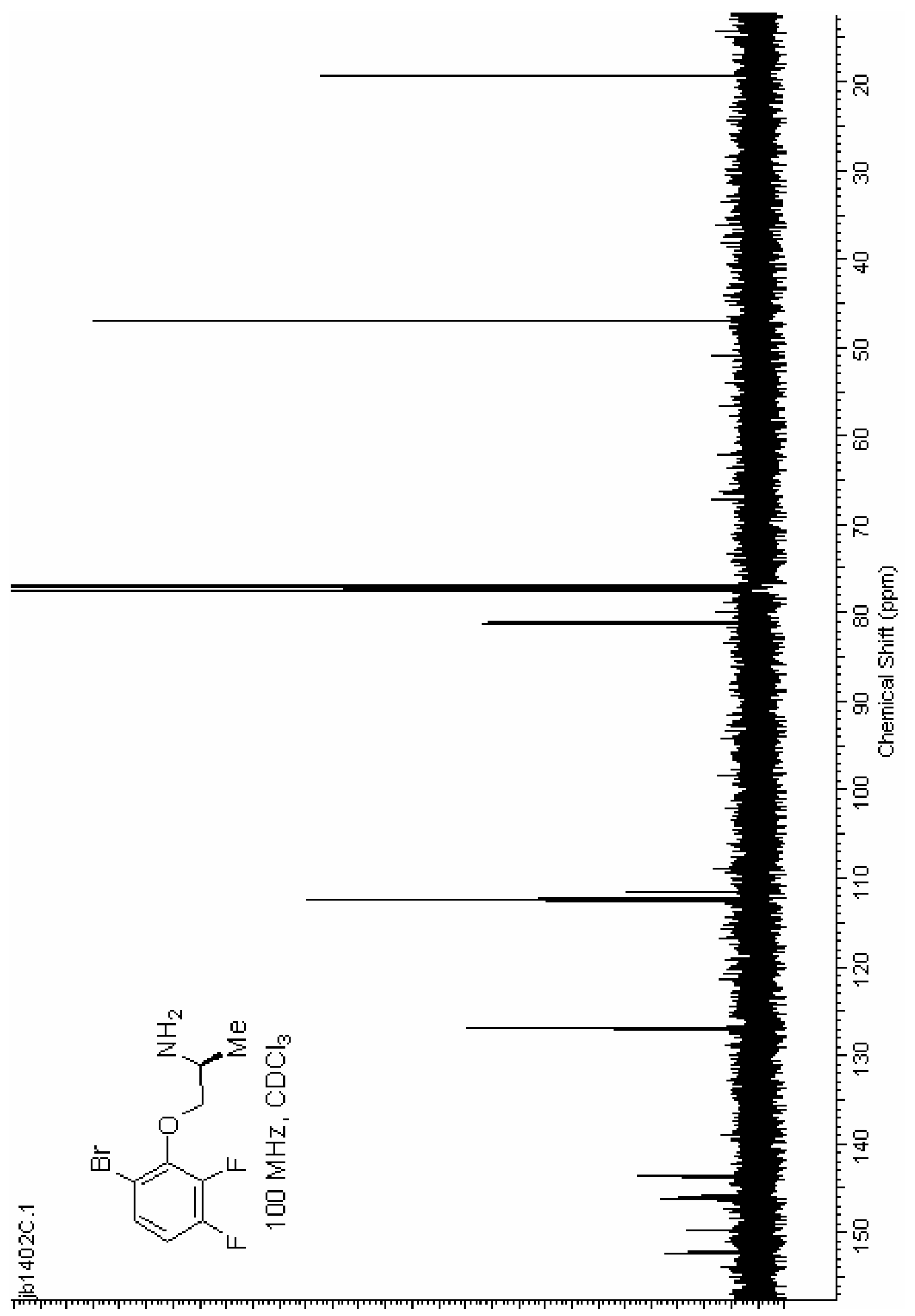




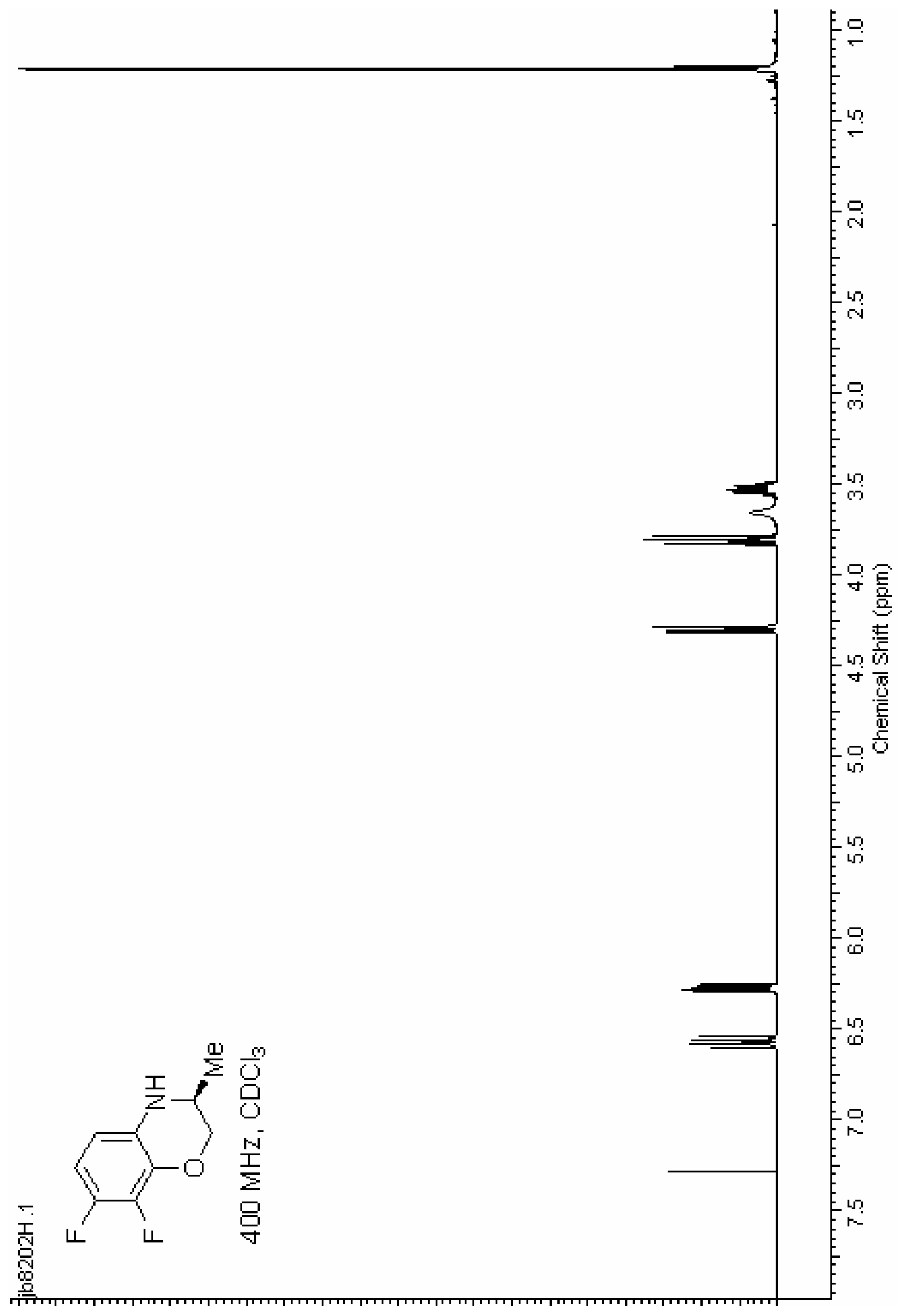




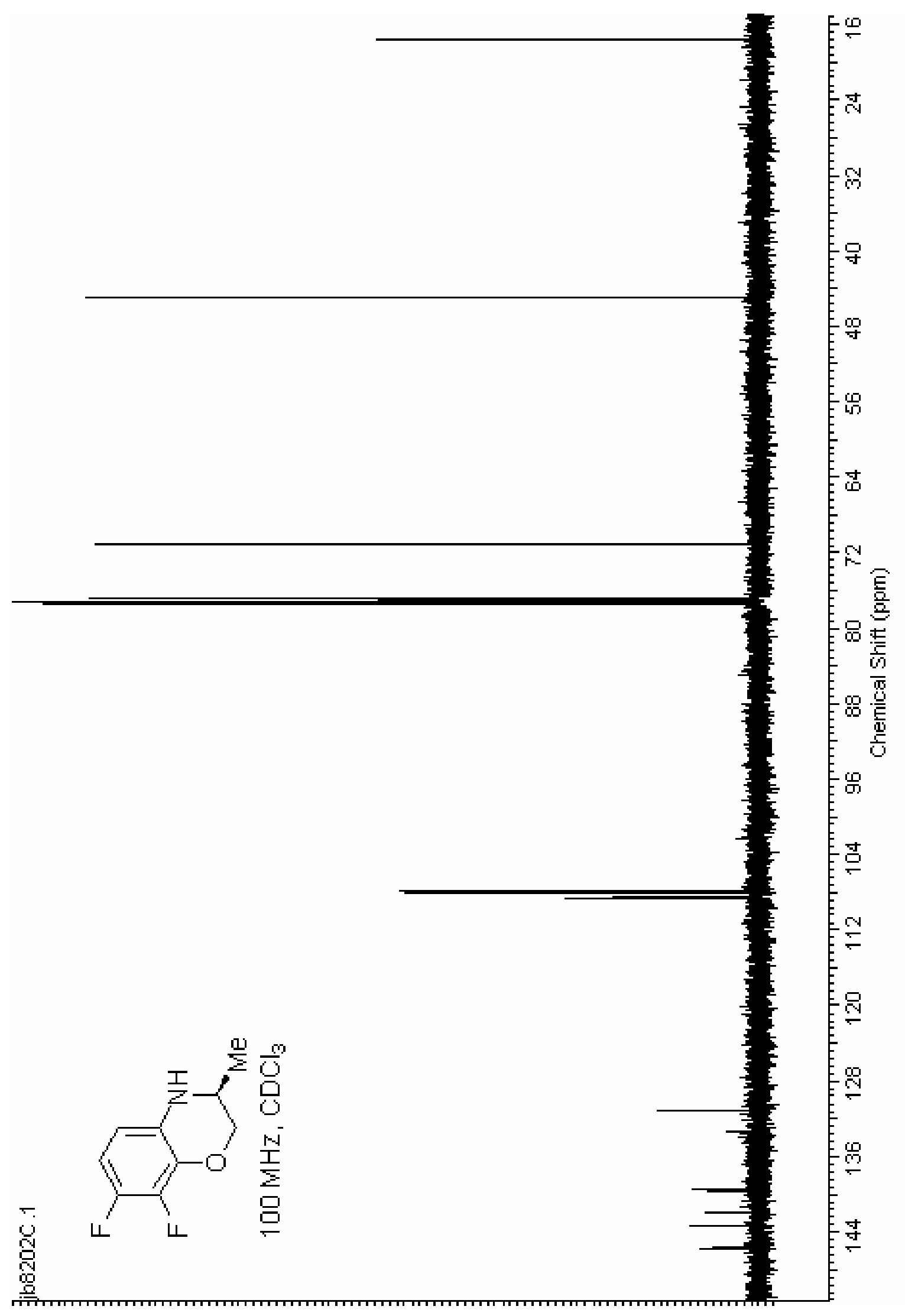

